\title{
Assimilating Synthetic Land Surface Temperature in a Fully Coupled Land-Atmosphere Model
}

\author{
DiSSERTATION \\ zur Erlangung des Doktorgrades \\ der Naturwissenschaften
}

vorgelegt beim Fachbereich 11

der Johann Wolfgang Goethe-Universität

in Frankfurt am Main

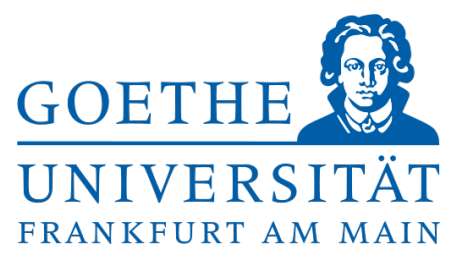

Von

Christine Sgoff

aus Frankfurt am Main

Frankfurt 2020

(D 30) 
vom Fachbereich 11 (Geowissenschaften/Geographie) der

Johann Wolfgang Goethe-Universität als Dissertation angenommen.

Dekan: Prof. Dr. Georg Rümpker

Gutachter: Prof. Dr. Juerg Schmidli

Prof. Dr. Roland Potthast

Datum der Disputation: 16.06.2021 




\section{Abstract \\ Assimilating synthetic land surface temperature in a fully coupled land-atmosphere model}

The weather of the atmospheric boundary layer significantly affects our life on Earth. Thus, a realistic modelling of the atmospheric boundary layer is crucial. Hereby, the processes of the atmospheric boundary layer depend on an accurate representation of the land-atmosphere coupling in the model. In this context the land surface temperature (LST) plays an important role. In this thesis, it is examined if the assimilation of LST can lead to improved estimates of the boundary layer and its processes.

To properly assimilate the LST retrievals, a suitable model equivalent in the weather prediction model is necessary. In the weather forecast model of the German Weather Service used here, the LST is modelled without a vegetation temperature. To compensate for this deficit, two different vegetation parameterizations were investigated and the better one, a conductivity scheme, was implemented. In order to make optimal use of the influence of the assimilation of the LST observation on the model system, it is useful to pass on the information of the observation to land and atmosphere already in the assimilation step. For that reason, a fully coupled land-atmosphere prediction model was used. Therefore, the existing control vector of the assimilation system, a local ensemble transform Kalman filter, was extended by the soil temperature and moisture. In two-day case studies in March and August 2017, different configurations of the augmented assimilation system were evaluated based on observing system simulation experiments (OSSE).

LST was assimilated hourly over two days in the weakly and strongly coupled assimilation system. In addition, every six hours a free 24-hour forecast was simulated. The experiments were validated with the simulated truth (a highresolution model run) and compared against an experiment without assimilation. It was shown that the prediction of the boundary layer temperature, especially during the day, and the prediction of the soil temperature, during the whole day and night, could be improved. The best impact of LST assimilation was achieved with the fully coupled system. The humidity variables of the model benefited only partially from the LST assimilation. For this reason, covariances in the model ensemble were investigated in more detail. To check their compatibility with the high-resolution model run the ensemble consistency score was introduced. It was found that the covariances between the LST and the temperatures of the high-resolution model run were better represented in the ensemble than those between the LST and the humidity variables. 


\section{Kurzfassung}

\section{Assimilation synthetischer Landoberflächentemperaturen in ein vollständig gekoppeltes Land-Atmosphären-Modell}

Das Wetter innerhalb der atmosphärischen Grenzschicht bestimmt maßgeblich unser Leben auf der Erde. Daher ist eine realistische Modellierung der Grenzschicht von großer Bedeutung. Die Prozesse der Grenzschicht sind dabei von einer genauen Darstellung der Kopplung zwischen Land und Atmosphäre im Modell abhängig. In diesem Rahmen spielt die Landoberflächentemperatur (engl. land surface temperature, LST) eine wesentliche Rolle. In dieser Dissertation wird untersucht, ob die Assimilation der LST zu verbesserten Vorhersagen der Grenzschicht und ihrer Prozesse führen kann.

Um die LST nutzbringend zu assimilieren, ist ein geeignetes Modelläquivalent im Wettervorhersagemodell erforderlich. Im hier verwendeten Wettervorhersagemodell des Deutschen Wetterdienstes wird die LST ohne eine Vegetationstemperatur modelliert. Um dieses Defizit auszugleichen, wurden zwei unterschiedliche Vegetationsparametrisierungen untersucht und die bessere, ein Konduktivitätsschema, implementiert. Um den Einfluss der Assimilation der LST-Beobachtung auf das Modellsystem optimal auszunutzen, ist es sinnvoll, die Informationen der Beobachtung bereits im Assimilationsschritt an Land und Atmosphäre weiterzugeben. Aus diesem Grund wurde ein vollständig gekoppeltes Land-Atmosphären-Vorhersagemodell verwendet. Dazu wurde der bestehende Kontrollvektor des Assimilationssystems, ein lokaler EnsembleTransform-Kalman-Filter, um Bodentemperatur und -feuchte erweitert. In zweitägigen Fallstudien in März und August 2017 wurden auf Basis synthetisch erzeugter Beobachtungen (engl. observing system simulation experiment, OSSE) verschiedene Konfigurationen des erweiterten Assimilationssystems getestet.

Die LST wurde im schwach und stark gekoppelten Assimilationssystem stündlich über zwei Tage assimiliert. Zusätzlich wurde alle sechs Stunden eine freie Vorhersage mit einem Vorhersagehorizont von 24 Stunden simuliert. Die Experimente wurden mit der simulierten Wahrheit (einem hochaufgelösten Modelllauf) validiert und gegen ein Experiment ohne Assimilation verglichen. Dabei zeigte sich, dass die Vorhersage der Grenzschichttemperatur, insbesondere tagsüber, und die Vorhersage der Bodentemperatur, im gesamten Tagesverlauf, verbessert wurde. Die besten Ergebnisse wurden mit dem vollständig gekoppelten System erzielt. Die Feuchtevariablen des Modells profitierten nur teilweise von der LST-Assimilation. Aus diesem Grund wurden die Kovarianzen des Modellensembles eingehender untersucht. Um ihre Kompatibilität mit dem hochaufgelösten Modelllauf zu überprüfen, wurde der Ensemble-KonsistenzScore entwickelt. Es zeigte sich, dass die Kovarianzen zwischen der LST und den Temperaturen des hochaufgelösten Modelllaufs im Ensemble besser dargestellt wurden als jene zwischen der LST und den Feuchtevariablen. 


\section{Contents}

Abstract i i

Kurzfassung iii

$\begin{array}{lr}\text { Contents } & 2\end{array}$

1 Introduction $\mathbf{5}$

$1.1 \quad$ Background of Land Surface Temperature Assimilation . . . . . 6

1.2 Goals of this Thesis . . . . . . . . . . . . . . . . . . 8

1.3 Method - Observing System Simulation Experiment . . . . . . . 9

1.4 Outline . . . . . . . . . . . . . . . . . . . 10

2 Assimilation Framework 11

2.1 Ensemble Kalman Filter . . . . . . . . . . . . . . . . . . . . . . 12

2.2 KENDA . . . . . . . . . . . . . . . . . . . . . . . . . . . 14

2.3 Augmented Control Vector $\ldots \ldots \ldots$

\begin{tabular}{lll}
\hline & Nature Run & 18
\end{tabular}

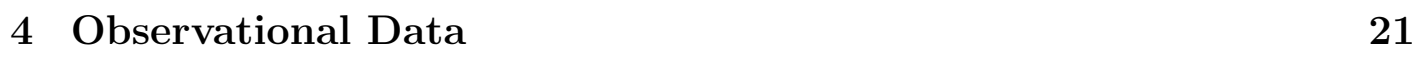

4.1 SEVIRI - Instrument . . . . . . . . . . . . . . . . . . . . . . . 21

4.2 Land Surface Temperature Retrieval . . . . . . . . . . . . . 21

$4.3 \quad$ Error Estimation of Land Surface Temperature Retrieval . . . . 23

5 Model Equivalent of Land Surface Temperature 24

5.1 Land Surface Temperature in COSMO Version 5.04(c) . . . . . 25

5.2 Canopy Scheme . . . . . . . . . . . . . . . . . 28

$5.3 \quad$ Skin Conductivity Scheme . . . . . . . . . . . . . . . . . . . 31

5.4 Comparison of Different Model Equivalent Implementations . . 32

\begin{tabular}{|ll}
6 & Synthetic Land Surface Temperature \\
\hline
\end{tabular}

7 Assimilation of Land Surface Temperature 40

7.1 Preparations . . . . . . . . . . . . . . . . . 40

$7.2 \quad$ Single Observation Experiments . . . . . . . . . . . . . . . . . . 41

7.2.1 Day: 27 March, 20171400 LT . . . . . . . . . . . . . . . 43

7.2 .2 Night: 27 March, 2017 0200 LT . . . . . . . . . . . . . . 45

7.3 Augmented Control Vector Tests _. . . . . . . . . . . . . . . 47

7.3 .1 Mean Error Evaluation . . . . . . . . . . . . . . . . . . . 49

$7.3 .2 \quad$ Root-Mean-Square Error Evaluation $\ldots . . . . . .53$ 
7.3 .3 Reduction of RMSE . . . . . . . . . . . . . . . . . . 55

$7.3 .4 \quad$ Impact on Surface and Near Surface Variables . . . . . . 59

7.3 .5 Evaluation of 24-hour Forecasts . . . . . . . . . . . . 62

7.3 .6 Summary . . . . . . . . . . . . . . . . 65

\begin{tabular}{|ll}
8 & Ensemble Consistency Score
\end{tabular}

8.1 Derivation . . . . . . . . . . . . . . . . . . . . 66

8.2 Lorenz 1963 System Experiments … . . . . . . . . . . . 70

$8.2 .1 \quad$ Experimental Setup . . . . . . . . . . . . . . . . 70

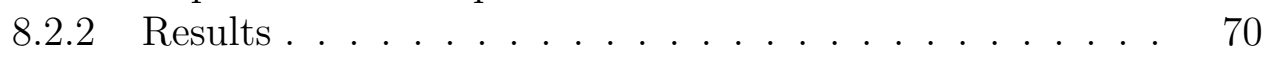

$8.2 .3 \quad$ Summary . . . . . . . . . . . . . . . . . . . . 74

$8.3 \quad$ Experiments with Synthetic Land Surface Temperature . . . . . 74

$8.3 .1 \quad$ Experimental Setup . . . . . . . . . . . . . 75

8.3 .2 Results . . . . . . . . . . . . . . . . . . . . 77

8.3 .3 Summary . . . . . . . . . . . . . . . . . 83

\begin{tabular}{llr}
\hline & Conclusions & $\mathbf{8 4}$
\end{tabular}

9.1 Discussion of Results . . . . . . . . . . . . . . . . . . . . . . . . . 85

9.2 Limitations and Outlook . . . . . . . . . . . . . . . . 88

\begin{tabular}{|ll}
\hline A Programming Lorenz 1963 System & 90
\end{tabular}

\begin{tabular}{ll}
\hline List of Figures & 96
\end{tabular}

\begin{tabular}{ll}
\hline List of Tables & 99
\end{tabular}

\begin{tabular}{ll}
\hline Bibliography & 100
\end{tabular}

$\begin{array}{ll}\text { Supplement: Deutsche Zusammenfassung } & 109\end{array}$ 




\section{Chapter 1}

\section{Introduction}

The planetary or atmospheric boundary layer (ABL) is the lowest layer of the terrestrial atmosphere. Almost the entire human activity takes place in this narrow atmospheric layer of about one to three kilometre height. Hence, weather events in this layer have a direct impact on life, society, agriculture, and economy. Here, the solar radiation reaches the land surface and the resulting turbulent energy fluxes make the ABL the most turbulent atmospheric layer and drive the physical interactions between the soil and the atmosphere. The processes of the ABL as well as the processes of the sub-surface soil are thus directly connected to the energy input at the soil. Hence, the development of the ABL strongly depends on the coupling between soil and atmosphere (Sandu et al., 2013; Holtslag et al., 2007; Koster et al., 2006). A realistic simulation of the ABL depends on whether the thermal coupling and the surface processes can be represented accurately in the model simulation (Trigo et al., 2015; Bosveld et al., 2014, Reichle et al., 2010).

Since the atmosphere is a chaotic system, it is not sufficient to only model its atmospheric processes correctly. In order to do forecasting also the initial state of the system must be determined as accurately as possible, because any deviations of the initial state from the unknown truth can grow exponentially. To put it clearly, even a perfect atmospheric forecast model cannot guarantee a correct weather prediction if the initial state contains uncertainties. To reduce errors of the initial model state, observations of the atmospheric state are used. This is done by combining observations and the predicted model state using data assimilation algorithms. There are several methods of data assimilation algorithms such as the variational method - 4DVAR (Talagrand and Courtier, 1987) - and the sequential ensemble-based methods such as - ensemble Kalman filter (Evensen, 1994) - to name the ones most regularly used. Within the context of the ABL, the combination of information from observed and simulated land surface fields can lead to a more representative estimate of land surface conditions and boundary layer processes (Ghent et al., 2010; Reichle et al., 2010; Han et al. 2013, Margulis and Entekhabi, 2003). In order to allow for a more accurate coupling of the land-atmosphere, it seems to be useful to introduce the information of observations into the atmosphere and the soil model. By now, mainly two possibilities to implement this are subjects of active research: weakly or strongly coupled land-atmosphere assimilation systems (Penny and Hamill, 
2017). In weakly coupled systems, the information of the observation is solely introduced into either the soil or the atmosphere model during the assimilation step. The observation information is then passed on to the respective other model in the next prediction step through the coupling between the land and atmosphere model. In strongly or fully coupled land-atmosphere assimilation systems, the observation is already introduced into the control state of both models simultaneously during the assimilation step. Therefore, the assimilation of land surface observations into a fully coupled land-atmosphere model is expected to lead to improvements of the initial model state and the forecasts of the coupling processes and the ABL.

The contributions to the energy budget at the land surface are well-known and include long- and shortwave radiation, sensible and latent heat flux, and soil heat flux. The land surface temperature (LST) is part of the surface energy budget and is thus one of the parameters that define the lower boundary condition of the atmosphere and the upper boundary condition of the soil. Hereby, LST determines the partitioning of sensible and latent heat flux and determines the upward thermal radiation. Therefore, improving the initial state of LST in weather forecast models has the potential to further enhance the representation of variables and fluxes that are correlated with the LST (Candy et al., 2017; Trigo et al., 2015; Santanello et al., 2013, Bosilovich et al., 2007). To observe the LST on large spatial scales, remote sensing observations derived by satellites are best suited (Houser et al., 2010). Consequently, a comprehensive LST retrieval can be derived from satellite measurements (Trigo et al., 2008a; Aires et al., 2004 Jin, 2004). Based on brightness temperatures observed by surface or near-surface sensitive channels of satellites. An enhancement of simulated LST can improve the simulation of brightness temperatures as well. In consequence, more brightness temperature measurements have the potential to be used within assimilation cycles (Trigo et al., 2015).

To also resolve the full diurnal cycle of LST, measurements of geostationary satellites are the best choice, because they measure with a high temporal resolution. The LST retrievals (Trigo et al., 2008a; Freitas et al., 2010) of the measurements of the Spinning enhanced visible and infrared imager (SEVIRI) on board Meteosat Second Generation (MSG) (Schmetz et al., 2002) are a promising option for LST assimilation, offering a temporal resolution of $\Delta t=15 \mathrm{~min}$ and a spatial resolution of $\Delta x \approx 5 \mathrm{~km}$ over central Europe.

\subsection{Background of Land Surface Temperature Assimilation}

LST retrievals already have been assimilated in offline land surface models to improve the energy budget at the land surface and the representation of land surface properties (Pipunic et al., 2008; Reichle et al., 2010; Ghent et al., 2010; Huang et al., 2008; Pinjosovsky et al., 2017; Han et al., 2013; Xu et al., 2018). 
For example, the LST observations from the International Satellite Cloud Climatology Project (ISCCP) were assimilated into two different land surface models, which led to enhancements in both models (Reichle et al., 2010). In a further study, Ghent et al. (2010) assimilated LST derived from the SEVIRI instrument into the Joint UK Land Environment Simulator (JULES). They were able to improve the simulated LST, soil moisture, as well as sensible and latent heat fluxes. Huang et al. (2008) combined the assimilation of LST retrievals derived from measurements of the Moderate Resolution Imaging Spectroradiometer (MODIS) into the Common Land Model (CoLM) with a simultaneous update of the leaf area index (LAI) by a MODIS LAI product, which led to an advanced profile of the modeled soil temperature. In a different approach Pinjosovsky et al. (2017) carried out LST assimilation to estimate enhanced land surface model parameters. They were able to improve the surface fluxes of the ORCHIDEE land surface model by estimating the most sensitive parameters to the LST. Han et al. (2013) assimilated MODIS LST in combination with microwave brightness temperature into the Community Land Model and found different impacts on soil moisture and soil temperature profiles. A joint assimilation was able to realize a more accurate soil moisture characterization under dry conditions, but under wet conditions the assimilation of brightness temperature outperforms the joint assimilation. Because the offline land surface models do not interact with atmospheric models, in all these studies, the impact of LST assimilation is restricted to soil and land surface variables.

Besides the assimilation of LST into offline land surface models, there are also studies which investigate the impact of LST assimilation on ABL models and weakly coupled land-atmosphere models (Boussetta et al., 2008; Zhang and Zhang, 2010; Tajfar et al., 2020a; Candy et al., 2017; Margulis and Entekhabi, 2003; Zhang et al., 2013). For example, McNider et al. (1994) assimilated LST tendencies derived from GOES satellite data into the surface energy budget of a meso-scale atmosphere model in order to improve the evapotranspiration term of the budget equation. They were able to get a better agreement with observations in a one- and three-dimensional test case. Margulis and Entekhabi (2003) combined the assimilation of radiometric surface temperature measurements with the assimilation of standard reference-level temperature and humidity. With their variational approach they enhanced the estimates of the land surface fluxes compared to a simple one-dimensional land surface-atmospheric boundary layer model. Improved surface flux estimates were also retrieved by Tajfar et al. (2020a). Here, the variational assimilation of LST into the soil heat diffusion equation (Bateni et al., 2013a b) was combined with the assimilation of reference-level air temperature and specific humidity into an ABL model (Tajfar et al., 2020b). Within a weakly coupled approach by Bosilovich et al. (2007) a bias corrected LST was assimilated into the land surface energy budget of a land surface model, which was used as a lower boundary condition for an atmospheric model. A positive impact was found on LST, sensible and latent heat flux, as well as on near-surface air temperature. As another example of a weakly coupled land-atmosphere LST assimilation, Candy et al. (2017) assimilated LST into a land surface model, whose updated soil temperature and moisture were used as forcing at the lower boundary of an atmosphere model. They found 
improvements in near-surface air temperature as well, but the enhancement of LST assimilation was dependent on the land cover type.

In conclusion, there is potential to benefit from assimilation of LST into soil and atmosphere models. To further enhance this impact of LST assimilation a possible step is to use a fully coupled land-atmosphere assimilation framework (Sgoff et al., 2020), where soil moisture and soil temperature are as well updated within the analysis step as the atmospheric variables. Another fully coupled land-atmosphere assimilation system has already been successfully implemented for soil moisture (Weather Research and Forecasting Model coupled with the NOAH land surface model, Lin and $\mathrm{Pu}$, 2018, 2019, 2020). However, the success of a fully coupled assimilation system depends on the cross-domain covariances between the coupled models (Smith et al., 2017, 2018; Sluka et al., 2016; Bannister, 2008a b; Ehrendorfer, 2007). Hence, it is crucial to also review the covariances between the observations and the analysed model variables.

\subsection{Goals of this Thesis}

With regard to the previous sections, there are still some steps to be taken on the way to a fully coupled land-atmosphere data assimilation system. The aim of this thesis is to contribute to this development.

Therefore, an Observing System Simulation Experiment (OSSE, Section 1.3) is performed to test the performance of LST assimilation into a fully coupled land-atmosphere system. As the coupled land-atmosphere forecast model the numerical weather prediction model of the Consortium for small-scale modelling model (COSMO-model, Baldauf et al., 2011) is chosen. The long-standing operational model of the German weather service (Deutscher Wetterdienst, DWD) consists of the atmospheric COSMO-model coupled with its own multilayered soil and vegetation model TERRA_ML (Doms et al., 2011; Schulz et al., 2016). Since the LST retrieval is employed as the assimilated observation within the experiments, it is important to have a suitable counterpart in the model. LST observed by satellites is a blended temperature from the soil surface temperature and the temperature from the vegetation above. Unfortunately, the COSMO-model provides only a soil surface temperature, which leads to a too weak diurnal cycle and too warm land surface temperatures at night (Vogel et al., 2015; Schulz et al., 2016; Shrestha et al., 2018). Thus, the first questions to be answered are:

- How to implement a vegetation/canopy temperature parameterization into the COSMO-model to predict a more accurate land surface temperature?

- Which implementation fits best with the LST retrieval derived from the remote sensing measurements to get a sufficient model equivalent?

To answer these questions, I collaborated with Jan-Peter Schulz and Jürgen Helmert (DWD) and evaluated two different promising parameterizations (Chapter 5). 
The Kilometre-scale Ensemble Data Assimilation scheme (KENDA, Schraff et al. 2016), which is based on the Local Ensemble Transform Kalman Filter (LETKF, Hunt et al., 2007) is chosen as the assimilation framework of this thesis to answer the questions:

- How does the assimilation of LST influence the atmospheric, the soil, and the land-atmosphere coupling values?

- Does the strongly coupled land-atmosphere assimilation system outperform the weakly coupled land-atmosphere assimilation system?

- How do background error covariances influence the impact of LST assimilation within the fully coupled land-atmosphere assimilation system?

The states of the forecast model, which are directly influenced by the observations in the assimilation step, are summarized in the control vector. By extending the KENDA control vector to soil variables, the strongly coupled landatmosphere assimilation system is realised. Thus, this thesis takes an important step towards the fully coupled land-atmosphere assimilation system. The strong coupling increases the potential to improve the lower atmospheric conditions in the assimilation step. This will further improve the processes on land surface and the prediction of the boundary layer.

\subsection{Method - Observing System Simulation Experiment}

To get first insights on the impact of the assimilation of the LST retrieval on the COSMO-model an observing system simulation experiment (OSSE) has been conducted. Masutani et al. (2010) give a comprehensive overview on the workings of OSSEs. Within this OSSE simulated LST observations are used as input for the data assimilation system to evaluate their impact on the coupled land-atmosphere system. Furthermore, the OSSE investigates the performance and workings of the coupled land-atmosphere data assimilation. The synthetic LST observations are derived from a simulation, which is assumed to be the truthful state of the system, from here on called the truth or nature run. The derived LST retrievals are assumed as perfect observations and are adjusted by realistic observational errors to better mimic real observations. The nature run of this study is a four-times higher resolved COSMO-model forecast and is further used to evaluate the analysis and forecasts of the OSSE. The motivation to conduct an OSSE is based on the following points:

- The truth is known and can be used for the evaluation of the experiments.

- Observations and their errors are well defined and bias-free.

- The evaluation of less observed variables, such as soil moisture, soil temperature and surface fluxes, is possible. 


\subsection{Outline}

Firstly, within Chapter 2 the coupled land-atmosphere assimilation framework is explained. The methodology of an OSSE motivates the order of the following chapters:

- Generation of a nature run (Chapter 3)

- The synthetic observations are derived from the nature run:

- The real LST retrieval is described (Chapter 4).

- The model equivalent of the LST retrieval is implemented and evaluated (Chapter 5).

- Based on that knowledge, the synthetic LST retrieval for the OSSE is generated (Chapter 6).

- The synthetic LST is assimilated into the fully coupled land-atmosphere system and the results of the LST assimilation on the land-atmosphere system are evaluated (Chapter 7).

In Chapter 8 an index is derived to allow a qualitative statement about the ensemble error covariances within the coupled land-atmosphere system. The conclusions and the outlook are presented in Chapter 9 .

This thesis, particularly the main parts of Chapter7, were the foundation of the publication by Sgoff et al. (2020). Thus, note that parts of the abstract, of Chapter 2 to 7, as well as parts of the conclusion in Chapter 9 are published in Sgoff et al. (2020). 


\section{Chapter 2}

\section{Assimilation Framework}

The main goal of data assimilation is to retrieve the best possible estimate of the atmospheric and soil state to initialize a forecast model properly. In there, observations of the real atmospheric state are combined with short-range model forecasts taking into account the error of both. Hereby, the forecast model provides a continuous and high resolution estimate of the atmospheric and soil state, but the forecast suffers from parameter and forcing errors, simplified physics, and insufficient model resolution. In contrast, the observations provide a picture of reality, but are less frequent in space and time. Furthermore, measurement errors and representativeness errors have to be considered for the observations. The combination of the information of both by data assimilation algorithms enables an improved initial state for the forecast model. Hence, the estimation of land surface processes can benefit from land surface observations, such as LST, by data assimilation (Houser et al., 2010).

To keep the mathematics of the data assimilation consistent within this work the following notation is used:

- lowercase bold symbols refer to vectors $\left(\mathrm{x}^{\mathrm{b}}, \mathrm{x}^{\mathrm{a}}, \ldots\right)$

- uppercase bold symbols refer to matrices $\left(\mathbf{X}^{\mathrm{b}}, \mathbf{R}, \ldots\right)$

- uppercase italic letters refer to operators $(H, M, \ldots)$.

The atmospheric state vector $\mathrm{x}^{\mathrm{b}}$ describes the relevant properties of the atmosphere, like temperature, pressure, wind, and humidity at each model grid point. The observation vector $\mathbf{y}$ summarizes observations of the real atmosphere, like the synthetic LST. In combination with their errors, both, the model state and the observations, contain information about the true state of the atmosphere $\mathbf{x}(t)$. To find the best estimate for a trajectory of the true state $\{\mathbf{x}(t)\}$ over a certain time period $t_{j}$ with $j=1, \ldots, n$, a common approach is to minimize the cost function $J(\mathbf{x})$, which takes the model state and its uncertainty $\mathbf{P}^{\mathrm{b}}$, and the observation and the observational uncertainty $\mathbf{R}$ into account:

$$
\begin{aligned}
J(\{\mathbf{x}(t)\})= & {\left[\mathbf{x}(t)-\mathbf{x}^{\mathrm{b}}\right]^{T}\left(\mathbf{P}^{\mathrm{b}}\right)^{-1}\left[\mathbf{x}(t)-\mathbf{x}^{\mathrm{b}}\right] } \\
& +\sum_{j=1}^{n}\left[\mathbf{y}_{j}-H_{j}\left(\mathbf{x}\left(t_{j}\right)\right)\right]^{T}\left(\mathbf{R}_{j}\right)^{-1}\left[\mathbf{y}_{j}-H_{j}\left(\mathbf{x}\left(t_{j}\right)\right)\right] .
\end{aligned}
$$

Here $\mathbf{x}^{\mathrm{b}}$ refers to the background estimate vector or so-called first guess, $\mathbf{y}_{j}$ refers to the observation vector at each time step $j$, and $H_{j}\left(\mathbf{x}\left(t_{j}\right)\right)$ is the model state 
projected to observation space using the forward observation operator $H_{j}$. If the uncertainty of the model state is large in comparison to the uncertainty of the observation, the solution of the minimization $\mathrm{x}^{\mathrm{a}}$, the so-called analysis, resembles the observation. If the uncertainty of the observation is large compared to the uncertainty of the model, the background estimate is nearly unchanged and hence the analysis is similar to the model.

\subsection{Ensemble Kalman Filter}

Within this study an ensemble Kalman filter Kalman, 1960; Evensen, 1994; Burgers et al. 1998) is used as assimilation algorithm. An ensemble Kalman filter is a sequential technique to find a solution that minimizes a cost function (Equation 2.1). Its core idea is to find a model state, which minimizes the departures between the observations and the model estimate under consideration of their respective errors. One property of the Kalman filter (Kalman, 1960) is that within an assimilation cycle both the state and its covariance matrix are updated. Any kind of Kalman filter consists of two steps. The first step is the forecast step, wherein the model state, the former analysis $\mathbf{x}_{j-1}^{a}$, and its associated covariance matrix are transported in time by the forecast operator $M_{t_{j-1}, t_{j}}$ to the next time step $t_{j}$, where the an observation becomes available

$$
\mathbf{x}_{j}^{b}=M_{t_{j-1}, t_{j}}\left(\mathbf{x}_{j-1}^{a}\right) .
$$

As the model state itself, its covariance matrix also has to be transported in time, but the forecast operator of an error covariance matrix is more difficult to determine. To reduce computational requirements resulting from integrating the error covariance matrix in time, it is useful to transport an ensemble of model states in time. By integrating an ensemble of model states forward in time, the covariance matrix and the model state are integrated simultaneously because the error covariance matrix $\mathbf{P}^{\mathrm{b}}$ can be calculated from the model state ensemble (Evensen, 1994; Burgers et al., 1998; Evensen, 2003). In the ensemble Kalman filter framework the model state is defined as the ensemble mean $\overline{\mathbf{x}}^{\mathrm{b}}$. The second step of each kind of Kalman Filter is the analysis step, where the model state $\mathbf{x}_{j}^{b}$, the observations $\mathbf{y}$ and their associated covariances, $\mathbf{P}_{j}^{\mathrm{b}}$ and $\mathbf{R}_{j}$, are combined to the new and hopefully improved model state $\mathbf{x}_{j}^{a}$.

Figure 2.1 illustrates the forward and analysis step of the Kalman filter schematically. Because all quantities of the analysis step are valid at time step $j$, the time index is omitted for the following description of the analysis step. The ensemble Kalman filter is based on the traditional Kalman filter analysis equations

$$
\begin{aligned}
\mathbf{x}^{\mathrm{a}} & =\mathrm{x}^{\mathrm{b}}+\mathbf{K}\left(\mathbf{y}-\mathbf{H} \mathbf{x}^{\mathrm{b}}\right) \\
\mathbf{P}^{\mathrm{a}} & =(\mathbf{I}-\mathbf{K} \mathbf{H}) \mathbf{P}^{\mathrm{b}},
\end{aligned}
$$

but the weighting matrix $\mathbf{K}$, also so-called Kalman gain matrix, is derived from the error covariances provided by the ensemble of model states (Evensen, 1994; Burgers et al., 1998)

$$
\mathbf{K}=\mathbf{P}^{\mathrm{b}} \mathbf{H}^{T}\left(\mathbf{H} \mathbf{P}^{\mathrm{b}} \mathbf{H}^{T}+\mathbf{R}\right)^{-1}
$$




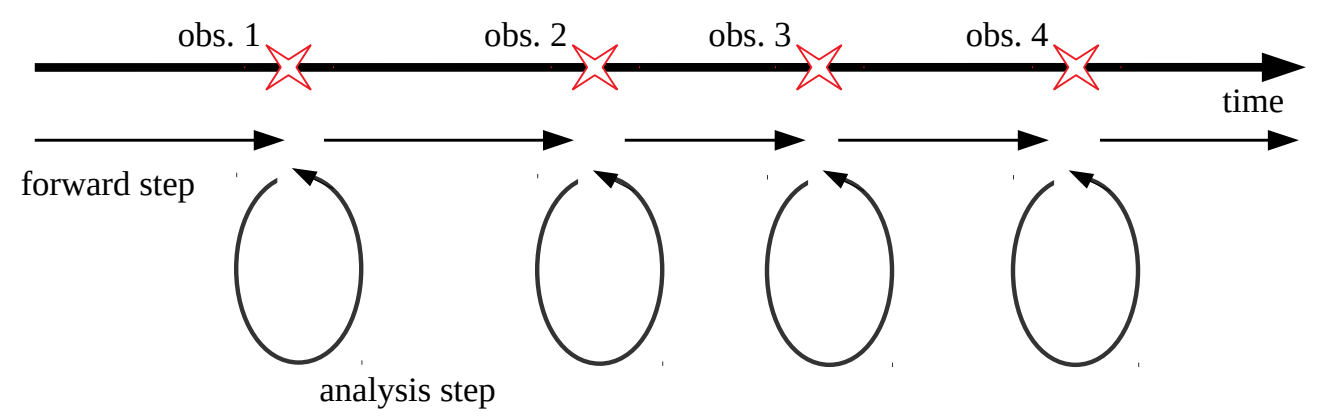

Figure 2.1: Schematic illustration of the Kalman filter work flow. The model state is forwarded in time towards the next observation. At each observation time (red cross) an analysis step is performed and a new initial state is generated to start the next forecast step.

Here, the linear observation operator $H$ is the matrix $\mathbf{H}$ relating the unknown true model state $\mathbf{x}^{\mathrm{t}}$ to the observations $\mathbf{y}$ combined with the measurement error $\epsilon$ :

$$
\mathbf{y}=\mathbf{H x}^{\mathrm{t}}+\epsilon .
$$

The observation error covariances $\mathbf{R}$ are based on the knowledge about and the estimation of measurement and representativeness errors. The background $\mathrm{x}^{\mathrm{b}}$, also known as first guess, is estimated by the sample mean of the background ensemble $\left\{\mathrm{x}^{\mathrm{b}(i)}: i=1,2, \ldots, k\right\}$ (Evensen, 2003, Hunt et al., 2007)

$$
\overline{\mathbf{x}}^{\mathrm{b}}=k^{-1} \sum_{i=1}^{k} \mathrm{x}^{\mathrm{b}(i)}
$$

As well as the background, the analysis $\mathbf{x}^{\mathrm{a}}$ is estimated by its sample mean

$$
\overline{\mathbf{x}}^{\mathrm{a}}=k^{-1} \sum_{i=1}^{k} \mathrm{x}^{\mathrm{a}(i)} .
$$

The calculation of the analysis ensemble is described in Section 2.2. The sample error covariance matrices of the first guess $\mathbf{P}^{\mathrm{b}}$ and of the analysis $\mathbf{P}^{\mathrm{a}}$ are estimated by

$$
\begin{aligned}
& \mathbf{P}^{\mathrm{b}}=(k-1)^{-1} \sum_{i=1}^{k}\left(\mathbf{x}^{\mathrm{b}(i)}-\overline{\mathbf{x}}^{\mathrm{b}}\right)\left(\mathbf{x}^{\mathrm{b}(i)}-\overline{\mathbf{x}}^{\mathrm{b}}\right)^{T} \\
& \mathbf{P}^{\mathrm{b}}=(k-1)^{-1} \mathbf{X}^{\mathrm{b}}\left(\mathbf{X}^{\mathrm{b}}\right)^{T}
\end{aligned}
$$

and

$$
\begin{aligned}
& \mathbf{P}^{\mathrm{a}}=(k-1)^{-1} \sum_{i=1}^{k}\left(\mathbf{x}^{\mathrm{a}(i)}-\overline{\mathbf{x}}^{\mathrm{a}}\right)\left(\mathbf{x}^{\mathrm{a}(i)}-\overline{\mathbf{x}}^{\mathrm{a}}\right)^{T} \\
& \mathbf{P}^{\mathrm{a}}=(k-1)^{-1} \mathbf{X}^{\mathrm{a}}\left(\mathbf{X}^{\mathrm{a}}\right)^{T}
\end{aligned}
$$

where $k$ is the ensemble size and the $k$ columns of the $\mathbf{X}^{\mathrm{b}}$ matrix and the $\mathbf{X}^{\mathrm{a}}$ matrix represent the deviations of the background $\mathbf{x}^{\mathrm{b}(i)}$ and analysis ensemble 
members $\mathbf{x}^{\mathrm{a}(i)}$ from the background $\overline{\mathbf{x}}^{\mathrm{b}}$ and analysis ensemble mean $\overline{\mathbf{x}}^{\mathrm{a}}$ Hunt et al., 2007):

$$
\begin{aligned}
& \mathbf{X}^{\mathrm{b}}(i)=\mathbf{x}^{\mathrm{b}(i)}-\overline{\mathbf{x}}^{\mathrm{b}} \\
& \mathbf{X}^{\mathrm{a}}(i)=\mathbf{x}^{\mathrm{a}(i)}-\overline{\mathbf{x}}^{\mathrm{a}} .
\end{aligned}
$$

\subsection{Kilometre-scale Ensemble Data Assimila- tion}

The data assimilation system used within this work is the kilometre-scale ensemble data assimilation system (KENDA, Schraff et al. (2016)), which is based on the local ensemble transform Kalman filter (LETKF) as derived by Hunt et al. (2007). The LETKF is an advanced version of the ensemble Kalman filter described in Section 2.1. The forward step is performed by the COSMO-model (Chapter 5) and simultaneously the model equivalents of the observations $\mathbf{H x}^{\mathrm{b}}$ are computed. Thus, the KENDA system is four-dimensional data assimilation scheme: a 4D-LETKF (Schraff et al., 2016). The analysis step is performed by the KENDA system hourly. For this purpose, the synthetic LST observations (Chapter 6) and the simulated first guess LST are used by KENDA to get the best estimate of the initial conditions for the next forecast step. The COSMOmodel is also used for 24 hour forecasts which are initialised every six hours starting from the analysis. Because the COSMO-model is a limited-area model boundary conditions are provided by the ICON-EU model (Zängl et al., 2015). Figure 2.2 illustrates the setup of the assimilation framework schematically.

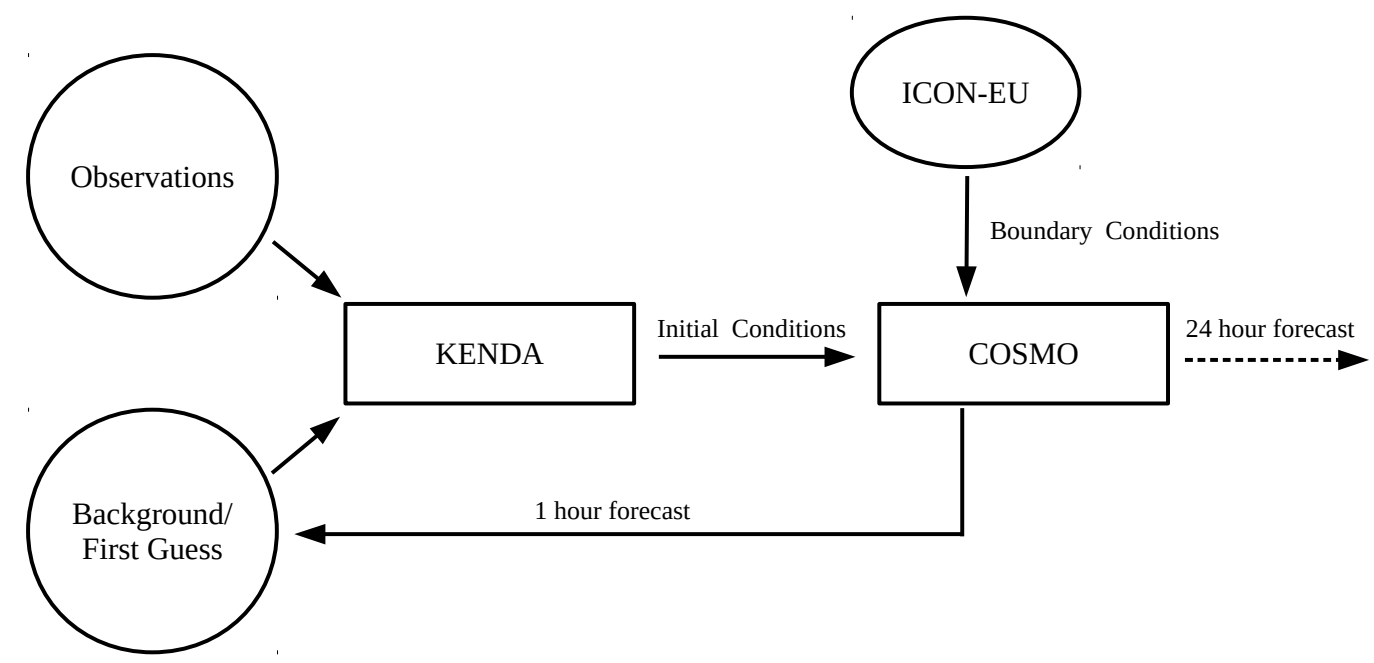

Figure 2.2: Scheme of the employed 4D-LETKF assimilation cycle. An analysis cycle is performed hourly. The observations, i.e. the synthetic LST retrieval, are valid for the same time as the background. The boundary conditions are provided by the European forecast of ICON-EU. At 0000, 0600, 1200, and 1800 UTC 24 hour forecasts are initialized from the analysis. 
The transformation of calculations to the $k$-dimensional ensemble space is the main feature of the LETKF (Hunt et al., 2007). By this transformation computational efficiency is gained compared to calculations performed in the higher dimensional physical space of a weather forecast model. To find the best-fitting state, the cost function

$$
J(\mathbf{w})=(k-1)\left(\mathbf{w}^{T} \mathbf{w}\right)+\left[\mathbf{y}^{0}-H\left(\overline{\mathbf{x}}^{\mathrm{b}}-\mathbf{X}^{\mathrm{b}} \mathbf{w}\right)\right]^{T} \mathbf{R}^{-1}\left[\mathbf{y}^{0}-H\left(\overline{\mathbf{x}}^{\mathrm{b}}-\mathbf{X}^{\mathrm{b}} \mathbf{w}\right)\right]
$$

is minimized in ensemble space (Hunt et al., 2007; Szunyogh et al., 2008). Here, $\mathbf{w}$ is the $k$-dimensional vector of ensemble weights, which corresponds to the model state vector

$$
\mathbf{x}=\overline{\mathbf{x}}^{\mathrm{b}}+\mathbf{X}^{\mathrm{b}} \mathbf{w}
$$

$\mathbf{R}$ is the observation error covariance matrix and $\mathbf{y}^{0}$ is the observation vector. By choice, the nonlinear observation operator $H$ is linearly approximated by

$$
H\left(\overline{\mathbf{x}}^{\mathrm{b}}+\mathbf{X}^{\mathrm{b}} \mathbf{w}\right) \approx \overline{\mathbf{y}}^{\mathrm{b}}+\mathbf{Y}^{\mathrm{b}} \mathbf{w},
$$

with $\overline{\mathbf{y}}^{\mathrm{b}}$ as the background mean in observation space and the $k$ columns of $\mathbf{Y}^{\mathrm{b}}$ represent the deviations between the ensemble member in observation space $\mathbf{y}^{\mathrm{b}(i)}$ and the ensemble mean $\overline{\mathbf{y}}^{\mathrm{b}}$. With the linear approximation of the observation operator the cost function Equation 2.15 gives

$$
J(\mathbf{w})=(k-1)\left(\mathbf{w}^{T} \mathbf{w}\right)+\left[\mathbf{y}^{0}-\overline{\mathbf{y}}^{\mathrm{b}}-\mathbf{Y}^{\mathrm{b}} \mathbf{w}\right]^{T} \mathbf{R}^{-1}\left[\mathbf{y}^{0}-\overline{\mathbf{y}}^{\mathrm{b}}-\mathbf{Y}^{\mathrm{b}} \mathbf{w}\right] .
$$

If the analysis $\overline{\mathbf{w}}^{\mathrm{a}}$, which minimizes Equation 2.18, is found, then the solution of the cost function in model space is given by

$$
\overline{\mathbf{x}}^{\mathrm{a}}=\overline{\mathbf{x}}^{\mathrm{b}}+\mathbf{X}^{\mathrm{b}} \overline{\mathbf{w}}^{\mathrm{a}}
$$

with

$$
\overline{\mathbf{w}}^{\mathrm{a}}=\mathbf{P}_{\mathrm{w}}^{\mathrm{a}}\left(\mathbf{Y}^{\mathrm{b}}\right)^{T} \mathbf{R}^{-1}\left(\mathbf{y}^{0}-\overline{\mathbf{y}}^{\mathrm{b}}\right) .
$$

The analysis update of the background error covariances in ensemble space $\mathbf{P}_{\mathrm{w}}^{\mathrm{a}}$ leads to

$$
\mathbf{P}_{\mathrm{w}}^{\mathrm{a}}=\left[(k-1) \mathbf{I}+\left(\mathbf{Y}^{\mathrm{b}}\right)^{T} \mathbf{R}^{-1} \mathbf{Y}\right]^{-1}
$$

which is

$$
\mathbf{P}^{\mathrm{a}}=\mathbf{X}^{\mathrm{b}} \mathbf{P}_{\mathrm{w}}^{\mathrm{a}}\left(\mathbf{X}^{\mathrm{b}}\right)^{T}
$$

in the model space. The analysis ensemble to initialize the next forecast step is generated such that $\overline{\mathbf{x}}^{\mathrm{a}}$ is the ensemble mean and $\mathbf{P}^{\mathrm{a}}$ the covariance of the ensemble. To ensure that the analysis ensemble $\left\{\mathrm{x}^{\mathrm{a}(i)}: i=1,2, \ldots, k\right\}$ in model space satisfies these conditions the background mean is a linear combination of the background ensemble deviations weighted by $\mathbf{w}^{\mathrm{a}(i)}$ (Hunt et al., 2007)

$$
\mathbf{x}^{\mathrm{a}(i)}=\overline{\mathbf{x}}^{\mathrm{b}}+\mathbf{X}^{\mathrm{b}} \mathbf{w}^{\mathrm{a}(i)}
$$

with

$$
\begin{aligned}
\mathbf{w}^{\mathrm{a}(i)} & =\overline{\mathbf{w}}^{\mathrm{a}}+\mathbf{W}^{\mathrm{a}}(i) \\
\mathbf{W}^{\mathrm{a}} & =\left[(k-1) \mathbf{P}_{\mathrm{w}}^{\mathrm{a}}\right]^{1 / 2} .
\end{aligned}
$$


The KENDA system is a 4D-LETKF system. For each observation within the first guess interval a model equivalent is simulated at the appropriate time. Therefore, the weighting of ensemble members is based on observations measured over the whole first guess time window. The KENDA-system is operational since March 2017 at the DWD. Its forecast model is the COSMO-model and in the operational setup an ensemble of $k=40$ members is calculated. In this work, a 40-member ensemble is used as well. In addition to the 40 ensemble members, the KENDA-system also includes a deterministic run which employs the Kalman gain matrix from the LETKF (Zhang et al., 2004, Whitaker and Hamill, 2012, Harnisch and Keil, 2015, Schraff et al., 2016). To prevent the analysis ensemble from losing to much spread the analysis ensemble is perturbed after each assimilation step. In contrast, the deterministic run is not perturbed, therefore, it provides a more realistic representation of the atmospheric state than the ensemble. Furthermore, the deterministic run provides a better representation of moist processes, such as clouds and precipitation, than the ensemble mean, because these fields are smoothed in the analysis ensemble mean due to the averaging. The analysis $\mathbf{x}_{\mathrm{det}}^{\mathrm{a}}$ of the deterministic assimilation cycle is calculated by

$$
\mathbf{x}_{\text {det }}^{\mathrm{a}}=\mathbf{x}_{\text {det }}^{\mathrm{b}}+\mathbf{K}\left(\mathbf{y}^{0}-H\left(\mathbf{x}_{\text {det }}^{\mathrm{b}}\right)\right)
$$

with the Kalman gain matrix for the ensemble mean $\mathbf{K}=\mathbf{X}^{\mathrm{b}} \mathbf{P}^{\mathrm{a}}\left(\mathbf{Y}^{\mathrm{b}}\right)^{T} \mathbf{R}^{-1}$. To avoid spurious correlations due to sample noise given by the small ensemble size a spatial localization is implemented. This means that for each analysis grid point the weighting matrix takes only local observations into account. The localization is implemented by a localization of the observation error covariance matrix $\mathbf{R}$. Hereby, the entries of the inverse of $\mathbf{R}$ are multiplied by a factor which decreases with the increase of the distance between observation and analysis grid point. This factor is determined by the Gaspari-Cohn correlation function (Gaspari and Cohn, 1999). The localization length scale of the implemented Gaspari-Cohn correlation function depends on the observation system and differs between the horizontal and the vertical direction. The optimal localization depends on the density and the quality of the assimilated observations. Within this OSSE study, only the LST retrieval is assimilated. The LST retrieval depends in part on the land surface properties, which can change within a few meters to kilometers. That is why in this work the horizontal localization-scale is set to a constant value of $5.5 \mathrm{~km}$, which translates a localization cut-off at about $20 \mathrm{~km}$. The operational system has an adaptive localization scale depending on the effective number of observations, where the localization radius varies between $50 \mathrm{~km}$ and $100 \mathrm{~km}$ (Schraff et al., 2016).

The assimilation is performed on the analysis grid which is horizontally three times coarser than the model grid. This is possible because the weighting field of the analysis state $\left\{\mathbf{w}^{\mathrm{a}(i)}: i=1,2, \ldots, k\right\}$ varies smoothly over short distances. Therefore, the weighting field is computed on the coarse analysis grid and spatially interpolated onto the fine model grid afterwards to save computational time (Yang et al., 2009). The vertical coarse analysis grid of the atmosphere has 35 pressure levels with a higher level density close to the surface to better represent the covariances of variables within boundary layer, i.e. the experiments 
are more resolved than the operational setup with 30 vertical analysis levels. Within the soil the vertical analysis grid is equivalent to the model grid.

\subsection{Augmented Control Vector}

One important part of this work is the evaluation of the strongly coupled data assimilation in the land atmosphere system. To achieve the strong coupling, the control vector of the KENDA system is augmented by the prognostic soil moisture and soil temperature. Both soil variables are simulated by the COSMO-model's own vegetation and soil model TERRA_ML and are not part of the operationally implemented control vector. In addition to the operational set of atmospheric variables (temperature, wind, pressure and specific humidity), the soil temperature and the soil moisture can now be updated with each assimilation cycle. The vertical localization in the soil differs from the vertical localization of the atmosphere. As in the atmosphere the vertical localization is also implemented by the Gaspari-Cohn correlation function (Gaspari and Cohn, 1999), but here the localization-scale $L$ has been set to $0.2 \mathrm{~m}$. Thus, the impact of LST on the soil is visible in the upper five soil layers of TERRA_ML. This has been chosen because the upper five soil layers represent a soil depth of approximately $1 \mathrm{~m}$. Soil layers below a depth of $1 \mathrm{~m}$ are nearly unaffected by temperatures of the land surface within time scales of days. It has been found that a localization-scale of $L=0.2 \mathrm{~m}$ represents this behaviour satisfactory (Figure 2.3).

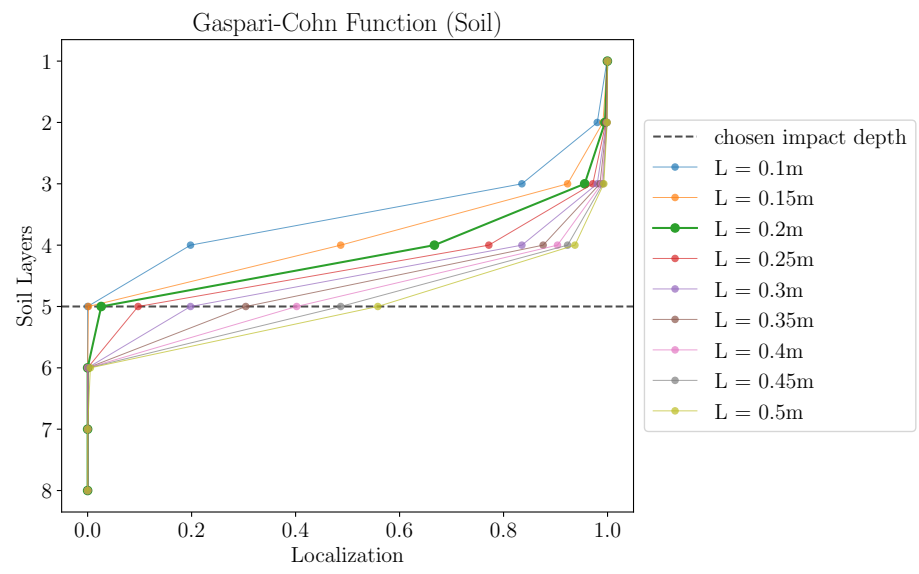

Figure 2.3: Evaluation of vertical localisation within the soil depending on different localization-scales $L$.

With the augmented control vector the assimilation of LST can now affect the atmosphere and the soil simultaneously. Because the variables of the control vector can be freely chosen, different control vector settings can be evaluated (Section 7.3). 


\section{Chapter 3}

\section{Nature Run}

Within this study two so-called nature runs were carried out. They were assumed to be the true state of the atmosphere and soil during the two different study periods. From these nature runs the synthetic LST observations were derived. Besides the generation of synthetic LST observations the nature runs were also used for the evaluation of the assimilation experiments.

Because the intermittent states of the nature runs were assumed to be the true states of the atmospheric and soil system they have to represent the atmospheric behaviour in a realistic way. Both nature runs were realized with the COSMO-model Version 5.4c including the skin conductivity scheme (Section (5.3), which was also used for the data assimilation system. To reduce the potential of overly optimistic results, a common way is to produce the nature run at a high spatial resolution and the data assimilation experiments at a lower spatial resolution. On that account the performed nature runs were of four times higher horizontal resolution than the forecasts of the data assimilation system and thus had a horizontal resolution of $0.7 \mathrm{~km}$. Consequently, the setup of the turbulence and radiation parameter schemes were adapted to the higher resolution. That means, that the radiation scheme was called every three forecast minutes - that is five times more often than in the operational COSMO-DE setup - and the TKE-based turbulence parameterisation took into account TKE advection and horizontal diffusion of TKE (Table 3.1 based on Blahak (2015)).

To enhance the impact of the assimilation of LST two periods with mainly clear-sky conditions in 2017 were chosen. The absence of clouds increases the available real and synthetic LST retrievals because their amount is limited by the cloud cover (Figure 3.1). Furthermore, in March 2017 the assimilation system KENDA (Schraff et al., 2016) became operational and provides a high-quality initial ensemble for the assimilation cycle. The first nature run is a four-day free-running simulation without any data assimilation in March 2017. These four days from March 25 to March 29, were chosen because of a large highpressure system over Europe, which lead to several clear-sky days (Figure 3.1a). The second nature run is a four-day period from August 26 to August 30, 2017 and also mainly cloudless (Figure 3.1b. 
Table 3.1: Adapted namelist setting of nature run, changes for turbulence parameterization are based on Blahak (2015).

\begin{tabular}{|l|l|l|}
\hline Name & Value & Definition \\
\hline \hline $\mathrm{dt}$ & 5 & time step in seconds \\
\hline dlat, dlon & 0.00625 & $\begin{array}{l}\text { horizontal grid spacing } \\
\text { [degree] }\end{array}$ \\
\hline nincrad & 36 & $\begin{array}{l}\text { interval between two } \\
\text { call of radiation } \\
\text { scheme }(36 \cdot d t=180 \text { s })\end{array}$ \\
\hline itype_turb & 3 & Prognostic TKE scheme \\
\hline lprog_tke & True & $\begin{array}{l}\text { TKE advection turned } \\
\text { on }\end{array}$ \\
\hline l3dturb & True & $\begin{array}{l}3 \mathrm{D} \text { turbulence scheme } \\
\text { turned on }\end{array}$ \\
\hline
\end{tabular}

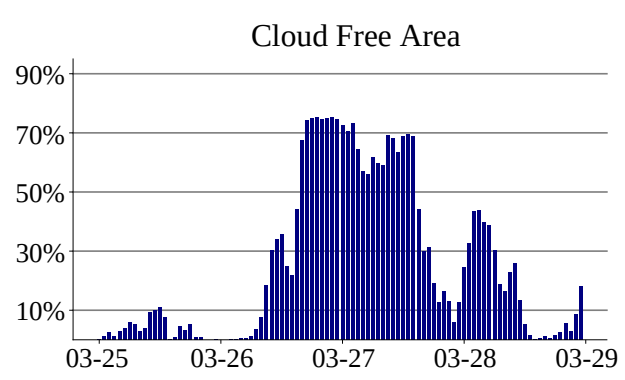

(a)

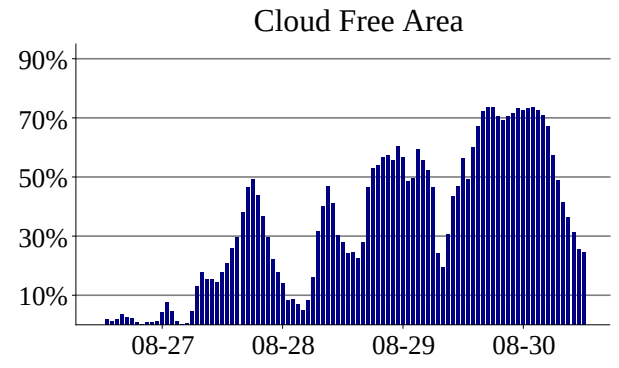

(b)

Figure 3.1: (a) Amount of cloud-free area of the nature run during the early spring experiment period (March 250000 UTC to March 290000 UTC, 2017) and (b) during the summer period (August 26 1200 UTC to August 301200 UTC, 2017).

The model domain of the experiments is located around Lindenberg, a DWD measurement site $70 \mathrm{~km}$ south-east from Berlin. The area covers the northeastern part of Germany and small parts of Poland and the Czech Republic (Figure 3.2). This part of the model domain is interesting because of the measurement site, whose data can be used for validation in a later study assimilating real LST retrievals. Furthermore it is advantageous, that the Earth surface height is mainly close to sea level, only in the south-eastern part of the experiment area some small mountains rise up to $1200 \mathrm{~m}$. High mountains increase the error of the LST retrieval. This is because, within one satellite pixel mountainous regions have a more heterogeneous surface height and characteristics and thus scatter the radiation more than flat terrain. Another disturbing factor, which occurs more frequently in mountainous regions, is snow. Snow and clouds reflect the solar radiation in a similar manner and because of that cloud detection errors may show up more often in mountainous areas. The main part of the model domain is covered by forests with some agricultural landscape in the south-west. Several small and big lakes are distributed around the area. Because of less memory requirements, the smaller model domain allows more experiments than experiments on the full COSMO-DE domain. Also the 
model domain of the experiment domain conveys more information than a singlecolumn model. The initialization and the boundary conditions for the nature run are gained from the COSMO-DE analysis interpolated to the smaller model domain and to the horizontal resolution of $700 \mathrm{~m}$.

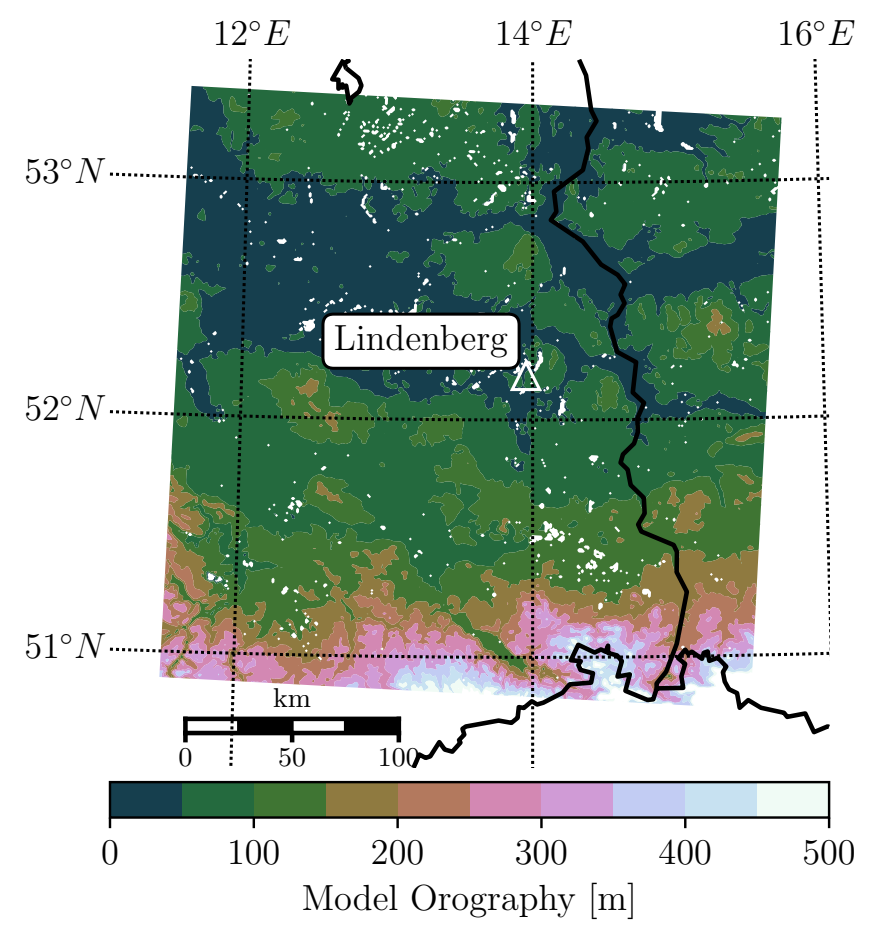

Figure 3.2: Surface height of model domain around the Lindenberg observatory (label and white triangle). White areas denote lakes. The white area on the bottom right is a mountainous region above $500 \mathrm{~m}$ height. 


\section{Chapter 4}

\section{Observational Data}

Because LST is a crucial variable of the energy budget at the land surface it was chosen as first observation to test the fully coupled land-atmosphere assimilation system. Surface sensitive satellite observations are used to derive LST retrievals. One LST product is provided by the Land Surface Analysis Satellite Application Facility (Land-SAF, Trigo et al., 2011). This LST retrieval (Trigo et al., 2008a; Freitas et al. 2010) is based on measurements of the Spinning Enhanced Visible and Infrared Imager (SEVIRI) on board of the geostationary Meteosat Second Generation (MSG) satellites (Schmetz et al., 2002).

\subsection{SEVIRI - Instrument}

MSG are a generation of European geostationary meteorological satellites. MSG satellites are spin stabilized and have a rapid imaging-repeat cycle of 15 minutes. The line-by-line scanning radiometer provides image data in twelve spectral channels over the whole MSG disk centered at $0^{\circ}$ longitude. The observed image data contains Africa, Europe and parts of Asia and South America with a resolution of about $3 \mathrm{~km}$ at subsatellite point. Over Germany, the resolution is about $5 \mathrm{~km}$. SEVIRI has thirteen spectral channels including a high-resolution visible channel. Eight of the channels are infrared (IR) channels in the range of $3.9 \mu \mathrm{m}$ to $13.4 \mu \mathrm{m}$ and four channels are visible and near-infrared (VNIR) channels in the range of $0.4 \mu \mathrm{m}$ to $1.6 \mu \mathrm{m}$. Each 15 minutes SEVIRI produces an image based on the last repeat cycle. Therefore, the LST product is also available every 15 minutes in near real time and offline.

\subsection{Land Surface Temperature Retrieval}

The LST retrieval, provided by the Land-SAF, is obtained from two channels within the thermal infrared atmospheric window: MSG/SEVIRI channel 9, centered at $10.8 \mu \mathrm{m}$ (IR10.8), and MSG/SEVIRI channel 10, centered at $12.0 \mu \mathrm{m}$ (IR12.0). Both channels provide surface and near surface information because, in the absence of clouds, they are most sensitive to the surface and the lower part of the troposphere. 
Clouds and aerosol layers can contaminate the observations and are a natural limitation of retrieval availability. They can block the signal from the surface or modify it due to absorption or scattering of the IR radiance. The absence of clouds is necessary to get an accurate LST retrieval. For that reason, the LST retrieval is derived for clear-sky pixels only. The clear-sky regions of the MSG disk are identified by the NoW Casting SAF, which provides a cloud mask for SEVIRI over Europe (NWC-SAF, 2016).

The retrieval itself is derived by a generalized split-window algorithm similar to the algorithm proposed by Wan and Dozier (1996) for the Advanced Very High Resolution Radiometer (AVHRR) and MODerate resolution Imaging Spectroradiometer (MODIS) data. The algorithm is adapted to SEVIRI measurements and the LST retrieval is estimated by a linear function of two topof-the-atmosphere (TOA) brightness temperatures of the split-window channels IR10.8 and IR12.0. The derivation has to take into account the spectral emissivities of different surface types, the atmospheric state, especially the water vapor content, and the orography. The surface orography can change with the viewing angle of the satellite. The surface emissivities are also derived by the Land-SAF. The estimation is based on the Vegetation Cover Method (VCM) approach (Caselles et al., 1997; Trigo et al., 2008b). Under the assumption of flat surfaces and the neglect of indirect radiation, which reaches the sensor, two types of emissivities are taken into account, the vegetation $\epsilon_{i, v}$ and bare ground $\epsilon_{i, g}$ band-emissivities on channel $i$. Both together are combined to one effective surface emissivity of each satellite pixel:

$$
\epsilon_{i, p i x e l}=\epsilon_{i, v} F C V+\epsilon_{i, g}(1-F C V) .
$$

The fraction of vegetation cover (FCV) is obtained from Land-SAF (Verger et al., 2009; Trigo et al., 2011) and used here as the weighting function of the two emissivities (Peres and DaCamara, 2005; Trigo et al., 2008b). The emissivity $\epsilon=0.5\left(\epsilon_{10.8}+\epsilon_{12.0}\right)$ and the difference between the two emissivities $\Delta \epsilon=\epsilon_{10.8}-\epsilon_{12.0}$ are used together with brightness temperature of both channels $\left(T b_{10.8}\right.$ and $\left.T b_{12.0}\right)$ to determine LST:

$$
\begin{aligned}
L S T= & \left(A_{1}+A_{2} \frac{1-\epsilon}{\epsilon}+A_{3} \frac{\Delta \epsilon}{\epsilon^{2}}\right) \frac{T b_{10.8}+T b_{12.0}}{2} \\
& +\left(B_{1}+B_{2} \frac{1-\epsilon}{\epsilon}+B_{3} \frac{\Delta \epsilon}{\epsilon^{2}}\right) \frac{T b_{10.8}-T b_{12.0}}{2}+C
\end{aligned}
$$

The coefficients $A_{i}, B_{i}$ and $C$, with $i=1,2,3$, of the split-window algorithm are determined on the basis of the LST calibration data by fitting Equation 4.2 to this database. The LST calibration data is based on global profiles of temperature, moisture and ozone (Borbas et al., 2005), which represent a wide range of atmospheric states under clear-sky conditions. This atmospheric profiles are assigned to different satellite viewing angles, land surface temperatures and emissivities to simulate a realistic range of measured brightness temperatures at TOA of SEVIRI channel 9 and 10. The simulation is done with MODerate spectral resolution atmospheric TRANSmittance algorithm (MODTRAN4) (Berk et al., 2000). 


\subsection{Error Estimation of Land Surface Temper- ature Retrieval}

The uncertainty of the LST retrieval is given by an observation error and a quality flag at each satellite pixel. The uncertainty of LST consists of several error sources: sensor noise, uncertainties from the surface emissivity estimation, inaccurate atmospheric water vapor profiles and errors due to the generalized split-window algorithm itself (Freitas et al., 2010). Another crucial error source is incorrect cloud detection. The used NWC-SAF cloud mask for SEVIRI has a validated accuracy on the order of $97 \%$ (NWC-SAF, 2016). That leads to a LST uncertainty between $1-2 \mathrm{~K}$ for most areas on the MSG disk, including all mentioned sources of errors (Freitas et al., 2010). The LST retrieval is validated against remotely sensed data of MODIS (Trigo et al., 2008a) and in-situ measurements (Göttsche et al., 2013). The comparison of LST derived from different remote sensing observations shows a better agreement over homogeneous terrain and at night-time. The overall differences between the observed data sets are lower or in the same range as the uncertainty of the LST retrieval (Trigo et al., 2008a; Göttsche et al., 2013). 


\section{Chapter 5}

\section{Model Equivalent of Land Surface Temperature}

The land surface temperature (LST) is a crucial component of the surface energy budget. LST influences the surface turbulent heat fluxes, the planetary boundary layer evolution and convection. As a central component of the surface radiative processes the accuracy of simulated LST depends on physical processes of clouds, radiation, soil moisture and precipitation (Bosilovich et al., 2007). In numerical weather models, LST can be seen as the radiative temperature of the model surface. Within this study the non-hydrostatic limited area COSMO-model (Baldauf et al., 2011) is used as forecast model. It has been developed by the Consortium for Small Scale Modeling (COSMO; see online at http://www.cosmo-model.org/), a group of meteorological services from Germany, Greece, Israel, Italy, Poland, Romania, Russia, and Switzerland. The following experiments are carried out with an extended COSMO 5.04c version. The representation of LST is kept fairly simple within the formerly operational COSMO-model. In there, the surface temperature refers to only two surface conditions: bare soil and snow. That means, all vegetated areas are also treated as bare soil and shading or insulating effects due to vegetation are not considered.

This simplification differs distinctly from the actual satellite observations. The corresponding channels of the SEVIRI instrument detect infrared radiation reflected and emitted by the top level of the land surface. Therefore, the observed temperature is a merged value of canopy, bare soil and snow temperature. The simple parameterization of LST in the COSMO-model is one potential reason why the modeled and the measured LST differ from each other. Figure 5.1 shows the averaged diurnal cycle of LST over Germany for four days in July 2015. The simulated LST shows a smaller amplitude of the daily cycle compared to the satellite retrieval. The underdeveloped amplitude of the diurnal LST cycle is a known problem of the COSMO-model (Vogel et al., 2015). Within the COSMO-model, LST set equal to the temperature of the topmost soil layer, hence it has a certain thickness and a higher heat capacity/conductivity to lower layers than the canopy top, where the satellite observations are located. The higher heat capacity is one possible reason for the under-expressed diurnal cycle.

To overcome these deficiencies and to get an improved model equivalent 
for the assimilation of LST two different vegetation schemes are reviewed in the following chapter. The structure of the chapter is as follows: Section 5.1 summaries the current representation of LST in the COSMO-model. Section 5.2 describes the vegetation scheme based on a prognostic vegetation temperature and Section 5.3 describes a vegetation scheme based on a skin layer above the soil surface. The comparison of both schemes and the current implementation is presented in Section 5.4 .

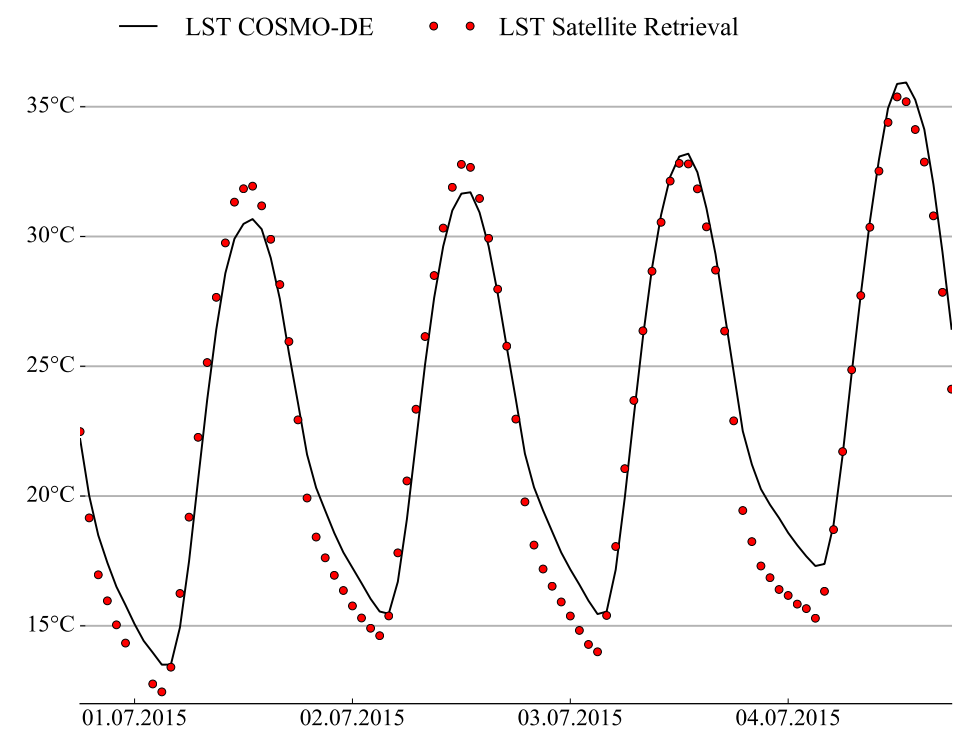

Figure 5.1: Domain-averaged skin temperature over Germany. Compared are the diurnal cycle of the LST satellite retrieval (red dots) and simulated LST (black line) over a four day period in July 2015. These four days (01.07.2015 - 04.07.2015) were chosen because the majority of Germany was cloud-free during this period. The domain average is calculated from all pixels, where both the model and the observation are cloud-free.

\subsection{Land Surface Temperature in COSMO Version 5.04(c)}

The starting point for the vegetation scheme experiments is COSMO version $5.04 \mathrm{c}$. The model includes a three-dimensional wind vector, temperature, pressure (deviation from a reference state), turbulent kinetic energy (TKE) and specific contents of water vapor, cloud water, cloud ice, rain, snow and graupel as prognostic variables. The prognostic equations are solved on an Arakawa C-grid and the coordinate system has rotated geographical coordinates with a generalized terrain-following height coordinate. The atmosphere is represented by a hybrid grid with terrain-following layers close to the surface of the Earth and horizontally flat model layers in the stratosphere above. The horizontal resolution of the model grid in the applied simulations is $2.8 \mathrm{~km}$ with 50 vertical 
layers. With this resolution deep moist convection is assumed to be be explicitly resolved.

The calculation of the LST itself is part of the multi-layer soil and vegetation model TERRA_ML (Doms et al., 2011; Schulz et al., 2016; Shrestha et al. 2018). Within TERRA_ML thermal and hydrological processes within and between the soil and a optional snow layer are calculated, respectively. For this purpose, a separate set of equations is provided and solved simultaneously. LST depends mainly on the thermal processes (Figure 5.2) and results from the upper boundary condition of the soil temperature. The evolution of temperature in the soil is based on the heat conduction equation

$$
\frac{\partial T_{\mathrm{so}}}{\partial t}=\frac{1}{\rho c} \frac{\partial}{\partial z}\left(\lambda \frac{\partial T_{\mathrm{so}}}{\partial z}\right)
$$

where $T_{\text {so }}$ is soil temperature, $\rho c$ is heat capacity and $\lambda$ is heat conductivity. Both $\rho c$ and $\lambda$ depend strongly on the eight different soil textures included in the soil model: sand, sandy loam, loam, loamy clay, clay, ice, rock and peat. Their properties are based on the Digital Soil Map of the World, which has a resolution of $10 \mathrm{~km}$ (Sanchez et al. 2009).

The soil is represented by a vertical layer structure. Here, seven active thermal layers at $0.01,0.03,0.09,0.27,0.81,2.43$, and $7.29 \mathrm{~m}$ and one inactive climate layer as constant lower boundary at $21.78 \mathrm{~m}$ depth are chosen. The temperature of the climate layer is the annual mean near-surface temperature. In reality, the annual temperature amplitude at $7.29 \mathrm{~m}$ is quite small, therefore a constant boundary layer below this depth may be safely used. The hydrological part of the soil has only six active layers based on the same layer structure as the thermal part (Figure 5.2). The lowest hydrological layer in a depth of $2.43 \mathrm{~m}$ accounts only for the downward gravitational transport, which contributes to the runoff.

The upper boundary condition of the soil model TERRA_ML is described by the coupling between the soil and the atmosphere. Radiation and sensible and latent heat fluxes contribute to the coupling between soil and atmosphere. LST is computed based on the energy budget calculation of the upper boundary condition. In its simplest form, the land surface temperature is determined by the bare soil temperature, neglecting impact of vegetation:

$$
\left(\frac{\partial T_{\mathrm{so}}}{\partial t}\right)_{k=1}=\frac{1}{\rho c(\Delta z)_{k=1}}\left[\lambda \frac{\left(T_{\mathrm{so}}\right)_{k=2}-\left(T_{\mathrm{so}}\right)_{k=1}}{\left(z_{\mathrm{m}}\right)_{k=2}-\left(z_{\mathrm{m}}\right)_{k=1}}+G_{\mathrm{s}}\right] .
$$

Here $k$ determines the level of a soil layer. The medium depth of a layer is $z_{\mathrm{m}}$ and the layer thickness is $\Delta z$. The upper soil boundary heat flux $G_{\mathrm{s}}$ is given by

$$
G_{\mathrm{s}}=H_{\mathrm{s}}+L_{\mathrm{s}}+R n_{\mathrm{s}}+G_{\mathrm{p}}+G_{\mathrm{snow}, \mathrm{melt}},
$$

with $H_{\mathrm{s}}$ as sensible heat flux, $L_{\mathrm{s}}$ as latent heat flux, and $R n_{\mathrm{s}}$ as net radiation at the surface. Effects of freezing rain and melting snowfall are represented by the term $G_{\mathrm{p}}$. The soil temperature changes due to snow melt processes are 


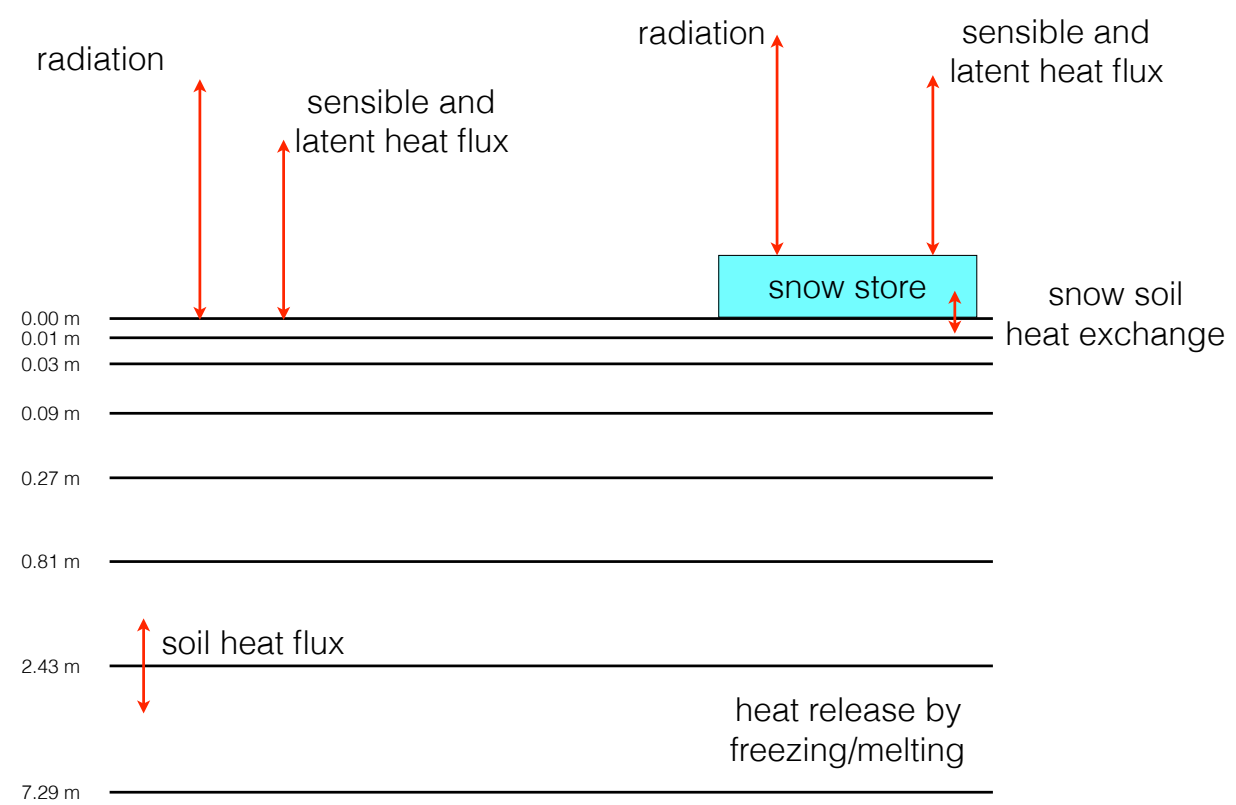

$21.87 \mathrm{~m}$

Figure 5.2: Layer structure of soil temperature and energetic processes considered by TERRA_ML. The size of the soil layers increases exponentially with depth. The top soil layer has a depth of $0.01 \mathrm{~m}$, meanwhile the undermost layer is of $14.58 \mathrm{~m}$ vertical width. Between the soil layers a vertical exchange of energy takes place via the soil heat flux. Further, heat can be released by freezing and melting processes. The upper boundary of the soil model is determined by the exchange fluxes with the atmosphere above. The bare soil and the snow cover interact with the atmosphere by radiation fluxes, sensible and latent heat fluxes. An energy exchange between snow store and top soil layer is also considered. Adapted from Doms et al. (2011). 
represented by $G_{\text {snow,melt }}$. The sensible heat flux $H_{\mathrm{s}}$ is taken from the surface layer parameterisation of the COSMO-model:

$$
H_{\mathrm{s}}=-\rho c_{\mathrm{p}} C_{\mathrm{h}}^{\mathrm{d}}\left|\boldsymbol{v}_{\mathrm{h}}\right|\left(\theta \pi_{\mathrm{s}}-T_{\mathrm{s}}\right)
$$

and depends on the bulk-aerodynamic transfer coefficient for turbulent heat exchange at the surface $C_{\mathrm{h}}^{\mathrm{d}}$, the atmospheric density $\rho$, the specific heat $c_{\mathrm{p}}$, and the absolute horizontal wind speed at the lowest atmosphere level, given by $\left|\boldsymbol{v}_{\mathrm{h}}\right|=\sqrt{u^{2}+v^{2}}$, where $u$ is the zonal and $v$ is the meridional wind speed. Furthermore, the sensible heat flux depends on the temperature difference between the bare soil surface temperature $T_{\mathrm{s}}$ and the temperature of the lowest atmospheric level $\theta \pi_{\mathrm{s}}$, derived from the potential temperature at the lowest atmospheric level $\theta$ and the Exner-pressure at the surface $\pi_{\mathrm{s}}$. Hereby, the surface temperature $T_{\mathrm{s}}$ influences the land surface flux and the coupling between atmosphere and soil. The latent heat flux is also determined by the surface layer parameterisation of the COSMO-model:

$$
L_{\mathrm{s}}=-\rho C_{\mathrm{q}}^{\mathrm{d}}\left|\boldsymbol{v}_{\mathrm{h}}\right|\left(q^{\mathrm{v}}-q_{\mathrm{s}}^{\mathrm{v}}\right),
$$

which depends on the bulk-aerodynamic coefficient for turbulent moisture transfer at the surface $C_{\mathrm{q}}^{\mathrm{d}}$, the specific latent heat $L_{\mathrm{v}}$, the atmospheric density, the absolute horizontal wind speed, and the difference between the specific humidity at the lowest atmospheric level $q^{\mathrm{v}}$ and the specific humidity at the surface $q_{\mathrm{s}}^{\mathrm{v}}$. In the current COSMO version $C_{\mathrm{h}}^{\mathrm{d}}=C_{\mathrm{q}}^{\mathrm{d}}$ is assumed. The computation of evapotranspiration is divided in bare soil evaporation (Schulz and Vogel, 2020) and transpiration from plants (Noilhan and Planton, 1989). The net radiation budget at the soil surface is determined by the $\delta$-two-stream method of Ritter and Geleyn (1992).

In general, the land surface temperature of TERRA_ML is a weighted temperature $T_{\mathrm{g}}$ of the bare soil temperature $T_{\mathrm{s}}$ and the snow surface temperature $T_{\text {snow }}$ :

$$
T_{\mathrm{g}}=\left(1-A_{\text {snow }}\right) T_{\mathrm{s}}+A_{\text {snow }} T_{\text {snow }} .
$$

\subsection{Canopy Scheme}

As Section 5.1 illustrated the COSMO-model lacks the impact of vegetation on the radiation budget and the sensible heat flux in the current operational model version. To take this effects into account a canopy temperature is one option to extend the existing TERRA_ML. One specific approach is the resistance approach from the AMBETI-model (Braden, 2012). The version examined within this study is developed for needle leaf forests and the prognostic canopy temperature equation is based on on the implementation of the land-surface scheme of the regional climate model developed by the Rossby Centre (Samuelsson et al., 2006). Jürgen Helmert (Deutscher Wetterdienst, DWD) implemented this parameterisation as a potential canopy scheme in an experimental version of the COSMO-model. 
The scheme includes surface fluxes and temperatures at the top surface of the canopy and at the canopy floor. While the soil of the COSMO-model is separated in several layers, the vegetation is considered as a single layer on top of the soil layers. Hence, the vegetation layer has an impact on the upper boundary condition of the soil and the lower boundary condition of the atmosphere. Like TERRA_ML, the vegetation model is a one-dimensional model and only vertical motions and transports are considered. The resistances to derive the aerodynamic transports are based on the idea of Ohm's Law in electricity, which is a common approach in agrometeorological models. Simply put, the flux density of a transport fluxes $\Phi$ equals the concentration difference $\Delta c$ divided by the aerodynamic resistance $r_{\mathrm{a}}$ :

$$
\Phi=-\frac{\Delta c}{r_{\mathrm{a}}} .
$$

Hereby, the aerodynamic resistance $r_{\mathrm{a}}$ substitutes the diffusivity $K$ of the free atmosphere, the vegetation, or other substances of the vegetation system by the integral of the reciprocal diffusivity:

$$
r_{\mathrm{a}}=\int \frac{1}{K} \mathrm{~d} z
$$

The diffusivity term $K$ represents the eddy diffusivity as well as the molecular diffusivity.

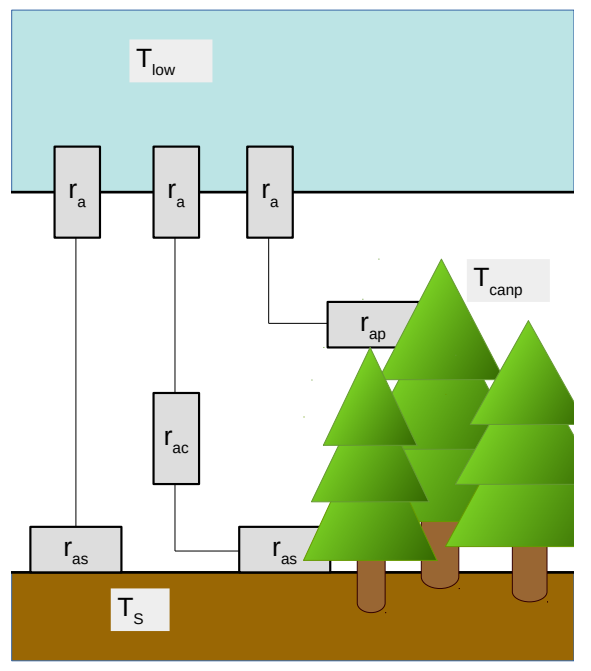

Figure 5.3: 'No-micro-climate' resistance approach of canopy scheme (Braden, 2012): architecture of the aerodynamic resistances for the sensible heat fluxes. The resistances are split in a resistance for the vertical turbulent transport above the canopy layer $r_{\mathrm{a}}$ and within the canopy $r_{\mathrm{ac}}$, and resistances for laminar transport at the canopy surface $r_{\text {ap }}$ and the soil surface $r_{\text {as }}$. $T_{\text {low }}$ is the temperature of the lowest atmospheric level, $T_{\text {canp }}$ is the temperature of the canopy top and $T_{\mathrm{s}}$ is the temperature of the soil surface. 
The main advantage of the resistance formulation is that fluxes in a network can be calculated with Kirchhoff's law. Here the 'no-micro-climate' version (Braden, 2012) of the vegetation model is implemented, which means that the turbulent vertical fluxes from the soil surface and from the plants are calculated separately.

The total flux at the top of the canopy $G_{\text {canp }}$ depends on the radiation budget $R n_{\text {canp }}$, the sensible heat flux $H_{\text {canp }}$ and the latent heat flux $L_{\text {canp }}$ at the top of the canopy

$$
G_{\text {canp }}=R n_{\text {canp }}-H_{\text {canp }}-L_{\text {canp }} \text {. }
$$

The time evolution of the temperature at the top surface of the canopy is determined from the total flux and the heat capacity of the forest canopy $C_{\text {canp }}$ (Samuelsson et al., 2006):

$$
\frac{\partial T_{\text {canp }}}{\partial t}=\frac{1}{C_{\text {canp }}} G_{\text {canp }}
$$

Here, $C_{\text {canp }}$ is calculated from the vegetative heat capacity $C_{\text {veg }}=2.7 \cdot 10^{3}$ $\mathrm{J} \cdot \mathrm{kg}^{-1} \cdot \mathrm{K}^{-1}$ (Verseghy et al. 1993$)$, the standing mass of needle leaf forest $W_{\text {veg }}$ (Verseghy et al., 1993), the volumetric heat capacity of the intercepted water $C_{\mathrm{w}} \rho_{\mathrm{w}}$ and the intercepted water $w_{\text {canp }}$ :

$$
C_{\text {canp }}=C_{\text {veg }} W_{\text {veg }}+C_{\mathrm{w}} \rho_{\mathrm{w}} w_{\text {canp }} .
$$

That means, that apart from the vegetation itself also the water collected by the plants $w_{\text {canp }}$ has an impact on the heat capacity and the canopy temperature evolution. The sensible heat flux $H_{\text {canp }}$ at the top of the canopy depends on the temperature difference between the canopy temperature $T_{\text {canp }}$ and the temperature of the lowest atmospheric level $T_{\text {low }}$. With the density $\rho$ and the specific heat $c_{\mathrm{p}}$ of air, that leads to

$$
H_{\text {canp }}=\rho c_{\mathrm{p}} \frac{T_{\text {low }}-T_{\text {canp }}}{r_{\text {ap }}+r_{\mathrm{a}}}
$$

The resistances of the sensible heat flux equation consist of the laminar resistance at the top of the canopy $r_{\text {ap }}$ and the resistance for the turbulent vertical transport between the lowest atmospheric level and the canopy surface $r_{\mathrm{a}}$.

The solar and thermal radiation at the top of the canopy depend on the vegetation coverage. There are several possibilities to describe the vegetation cover. Here the sky view fraction

$$
\chi=\exp (-0.5 \cdot T A I)
$$

is used, which is defined as the fraction of the sky seen by the ground under the canopy (Verseghy et al., 1993). The Transpiration Area Index TAI is derived from the Leaf Area Index (LAI) by $T A I=A_{\text {veg }} \cdot L A I$ with $A_{\text {veg }}$ as fractional vegetation cover. The LAI is a dimensionless quantity and defined by the leaf area per unit surface area. A high LAI can exceed the value one, if there are several leaf layers over one unit surface. A high LAI or TAI leads to a strong attenuation of the sky view fraction. High TAI and low $\chi$ are found, for example, 
for needle leaf forests. In contrast, bare soil areas, like deserts or glaciers, have a high sky view fraction value of one. If the vegetation cover is described by the sky view fraction $\chi$, the radiation budget of solar and thermal radiation is derived as follows

$$
\begin{aligned}
R n_{\text {canp }}= & (1-\chi)\left(1-\alpha_{\text {canp }}\right) S \downarrow+(1-\chi) \epsilon_{\text {canp }} \cdot \\
& \left(L \downarrow+\sigma\left(\left(1-A_{\text {snow }}\right) T_{\mathrm{s}}^{4}+A_{\text {snow }} T_{\text {snow }}^{4}-2 T_{\text {canp }}^{4}\right)\right),
\end{aligned}
$$

where $S \downarrow$ is downward short wave radiation, $L \downarrow$ is downward long wave radiation, $\alpha_{\text {canp }}$ is the albedo of the canopy, $\epsilon_{\text {canp }}$ is the canopy emissivity value, and $A_{\text {snow }}$ defines the fraction of the snow coverage. Thus the prognostic $T_{\text {canp }}$ (Equation 5.10) depends on a wide range of vegetation parameters due to different kinds of vegetation cover. For reasons of simplicity, here all parameters are adapted for needle leaf forests.

As already defined in Equation 5.6 the land surface temperature $T_{\mathrm{g}}$ is a composite temperature of the $T_{\mathrm{s}}$ and $T_{\text {snow }}$. But now also $T_{\text {canp }}$ is part of the weighted surface temperature:

$$
T_{\mathrm{g}}=\left(1-A_{\text {snow }}\right)\left[\chi T_{\mathrm{s}}+(1-\chi) T_{\text {canp }}\right]+A_{\text {snow }}\left[\chi T_{\text {snow }}+(1-\chi) T_{\text {canp }}\right] .
$$

\subsection{Skin Conductivity Scheme}

Another possible approach to overcome the current deficiencies of the COSMOmodel is a skin layer, as implemented by Schulz and Vogel (2020). The skin layer is a layer directly above the soil surface with no heat capacity (based on land surface model H-TESSEL (Viterbo and Beljaars, 1995; Verhoef and Vidale, 2012) of the European Centre for Medium-range Weather Forecasts (ECMWF)), which simulates the effects of different surface properties such as vegetation. For example, the soil below vegetated areas receives less radiative heating compared to bare soil areas. This insulating effect of vegetation can be represented by the skin layer. In the COSMO-model the skin layer approach is used for snow-free regions. Areas covered by snow are still represented by the snow parameterisation. The additional skin layer is defined by a skin conductivity $\Lambda_{\text {skin. }}$. This conceptual value provides the thermal connection between the skin level and soil surface based on Fourier's Law

$$
G_{\text {skin }}=\Lambda_{\text {skin }}\left(T_{\text {skin }}-T_{\mathrm{s}}\right) \text {. }
$$

The heat flux $G_{\text {skin }}$ is proportional to the magnitude of the temperature difference between the diagnostic skin level temperature $T_{\text {skin }}$ and the bare soil temperature $T_{\mathrm{s}}$. The forcing at the soil surface $G_{\text {skin }}$ is given by the sum of the radiation budget $R n_{\text {skin }}$, the sensible heat flux $H_{\text {skin }}$ and the latent heat flux $L_{\text {skin }}$ :

$$
G_{\text {skin }}=R n_{\text {skin }}-H_{\text {skin }}-L_{\text {skin }} .
$$

Various surface properties heat up or retain heat differently and should be represented with an appropriate heat conductivity. As simplification one useful mean value is used $\Lambda_{\text {skin }}=10 \mathrm{Wm}^{-2} \mathrm{~K}^{-1}$ (Schulz and Vogel, 2020). 


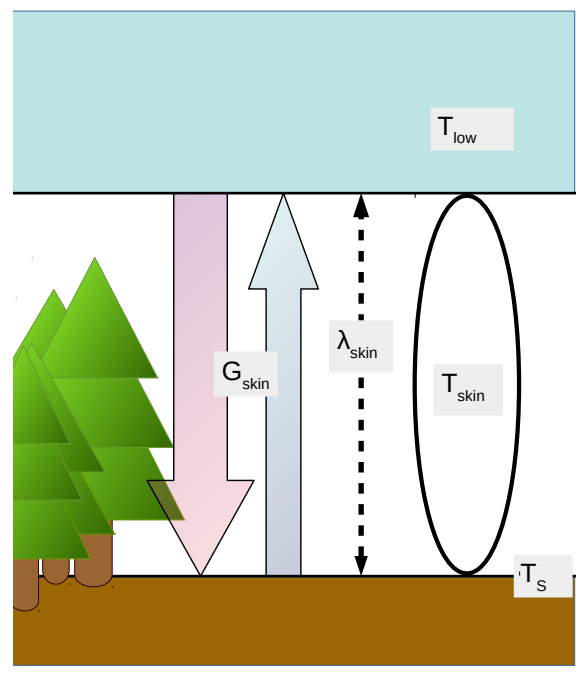

Figure 5.4: Skin conductivity layer scheme: schematic representation of the skin layer including the forcing at the soil surface $G_{\text {skin }}$ (Equation 5.17), the skin conductivity $\Lambda_{\text {skin }}$ and the connected temperatures of the soil surface $T_{\mathrm{s}}$ and the skin layer $T_{\text {skin. }} . T_{\text {low }}$ is the temperature of the lowest atmospheric layer, the layer above the skin layer.

The sensible heat flux $H$ is defined by the surface layer parameterisation, but uses $T_{\text {skin }}$ instead of $T_{\mathrm{s}}$

$$
H_{\text {skin }}=\rho c_{\mathrm{p}} C_{\mathrm{h}}^{\mathrm{d}}\left|\boldsymbol{v}_{\mathrm{h}}\right|\left(\theta \pi_{\mathrm{s}}-T_{\text {skin }}\right),
$$

where $C_{\mathrm{h}}^{\mathrm{d}}$ is the bulk-aerodynamic transfer coefficient for turbulent heat exchange at the surface, $c_{\mathrm{p}}$ is the specific heat, and $\rho$ is the density of air. The latent heat flux $L_{\text {skin }}$ and the net long- and shortwave radiation at the soil surface $R n_{\text {skin }}$ are implemented as in the soil model TERRA_ML (Section 5.1), but depend on the skin layer temperature $T_{\text {skin }}$. The same applies to Equation 5.6. now $T_{\mathrm{g}}$ is composite of $T_{\text {skin }}$ and $T_{\text {snow }}$ :

$$
T_{\mathrm{g}}=\left(1-A_{\text {snow }}\right) T_{\text {skin }}+A_{\text {snow }} T_{\text {snow }} .
$$

To summarize, the skin layer can be seen as a new upper boundary condition for the soil and as a new lower boundary condition for the atmosphere.

\subsection{Comparison of Different Model Equivalent Implementations}

For an appropriate comparison of the different implementations, a large sample of LST measurement data is necessary. Because the amount of observations depends mainly on the cloud cover, a clear-sky period over Germany is a good starting point. During summer of 2015 a period of three days with only little 
cloud cover was chosen. The period started on June 30 th and ended three days later on the 3th of July 2015. Especially, the first two days of the period are about up to $80 \%$ cloudless within the observational data and about $65 \%$ cloudless within the simulated data. The simulated data is declared as clear-sky, if the total cloud cover is less than $10 \%$ at a grid point. A $80 \%$ clear-sky area over the COSMO-DE domain (Germany and parts of neighboring countries) equals $\sim 47000$ usable observation points every 15 minutes. Figure 5.5 shows the evolution of cloud cover during the three-day period. The observations have even larger clear-sky areas than the COSMO-model. Both observations and the simulated cloud cover show the same trend. Two days of mainly clear-sky conditions followed by a cloudy third day.

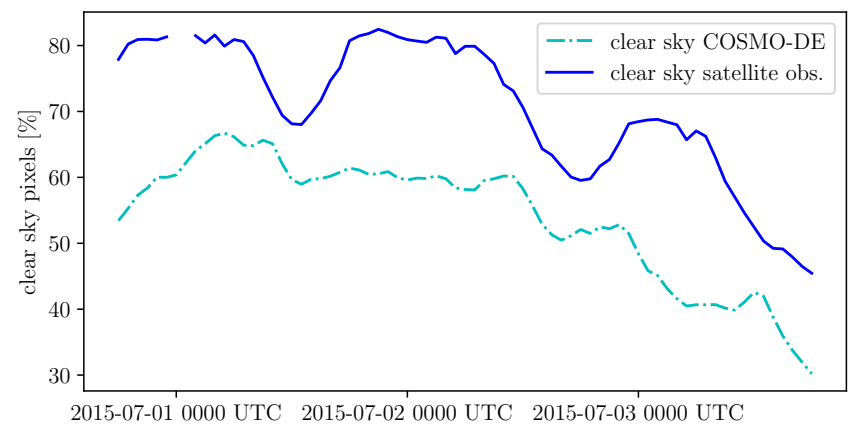

Figure 5.5: Evolution of clear-sky area over the COSMO-DE domain of satellite observations (blue, solid) and COSMO-model (light blue, dashed). The period is from the 30.06.2015 at 18:00 UTC to the 03.07.2015 18:00 UTC.

LST is strongly influenced by solar radiation. Clouds block incoming and outgoing radiation by absorption and reflection. Hence, LST decreases fast, if clouds cover the land surface. Due to different cloud fractions between the model and the observations significant deviations in near-surface temperatures can occur. Discrepancies due to different cloud fractions are possible, if the model contains clouds at points the LST retrieval shows a clear sky. Because the Land-SAF does not produce LST retrievals for cloudy pixels, observations are assumed as cloudless, if they are available. To get a reliable result the simulated temperature and the LST retrieval are only compared, if both are declared as cloudless.

The period of summer 2015 shows a clear diurnal cycle for simulated and measured LST (Figure 5.6). The LST observations have a stronger amplitude during day and night than the the simulated LST of the reference. The reference is based on COSMO Version 5.04c and does not contain any vegetation scheme for temperature. Hence, LST is derived from the temperature of the uppermost soil layer with a thickness of $1 \mathrm{~mm}$. Through the stronger coupling to the soil temperature the simulated LST of the reference has higher inertia than the observed LST, which causes the less distinct diurnal cycle. During 


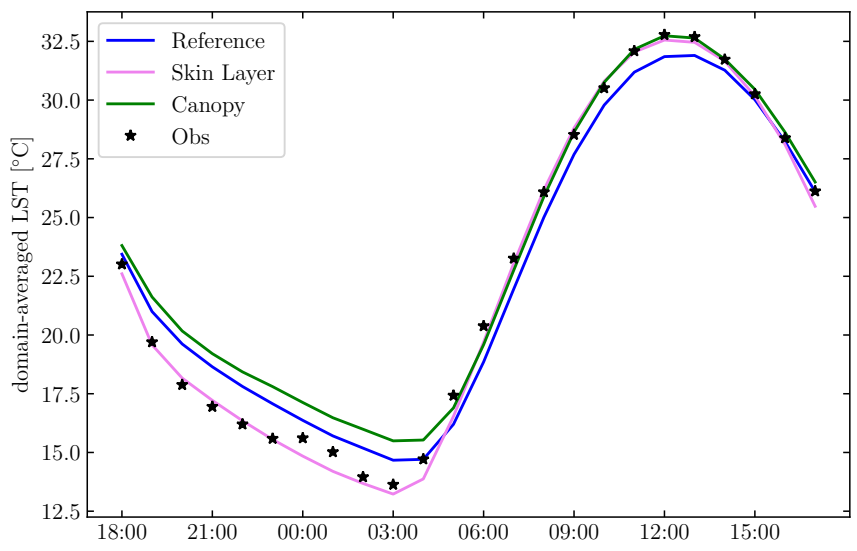

Figure 5.6: Domain averaged diurnal cycle of observed and simulated LST. The period starts at 30.06.2015 18:00 UTC and ends at 03.07.2015 18:00 UTC. The averaged LST retrieval is marked with black stars, the blue line shows the averaged COSMO-model, the green line shows the COSMO-model with the canopy scheme (section 5.2 and the purple line shows the COSMO-model with the skin layer scheme (section 5.3).

daytime, both experiments with a vegetation scheme fit better to the observations than the reference (Figure 5.6). During nighttime the skin conductivity scheme represents the observed LST considerably better than the reference and the experiment with the vegetation scheme.

A closer look at the model minus observation distribution (Figure 5.7) shows that the mean deviation from the observations of the reference and the experiment including the skin layer scheme are close to zero (both less than $0.25 \mathrm{~K}$ ). In contrast, the mean deviation of the experiment including the canopy scheme from the observations is higher and about $1 \mathrm{~K}$, what indicates a positive bias in LST. Figure 5.6 also shows, that the averaged diurnal cycle of LST with the canopy scheme is too warm, especially during night. The standard deviation of all LST experiments is similar and close to $2.3 \mathrm{~K}$. The skin layer scheme experiment has, compared to the other two experiments, a slightly smaller standard deviation.

Looking at the model minus observation distribution depending on the time of the day (Figure 5.8) a clear connection between daytime and the mean deviation is visible. The reference and the experiment including the canopy scheme show a warm model bias during night. The experiment including the skin layer scheme has a lower warm bias during night than the other simulations. All three simulations have cold bias during day. But both simulations with a vegetation scheme have a smaller daytime bias than the reference.

The three different options to simulate LST are described in Sections 5.1 to 5.3 . Experiments with all three different implementations have been 


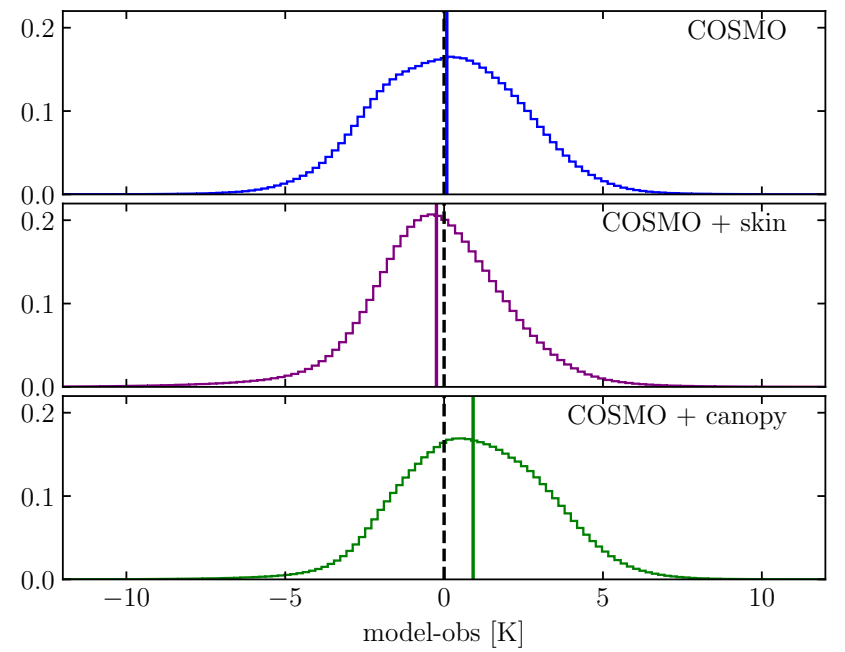

Figure 5.7: Distribution of the difference between COSMO-model and observation (LST retrieval) over the three day summer period 2015. Shown are the reference COSMO-model Version 5.04c without any vegetation scheme (blue), the canopy scheme, see section 5.2 , (green) and the skin layer scheme, see section 5.3. (purple).
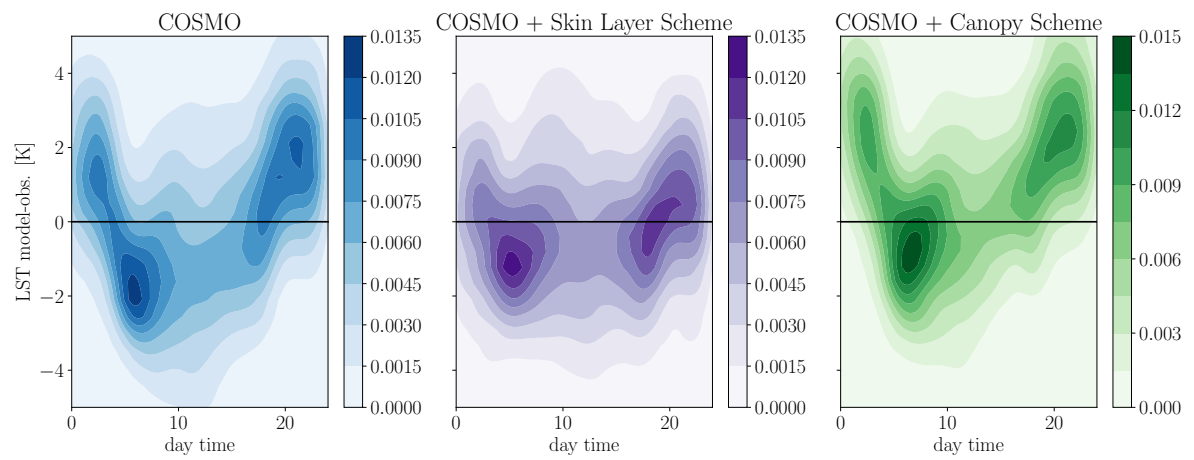

Figure 5.8: Simulated LST minus observed LST is shown against day time for all three model implementations. The distribution of reference minus observations throughout the day is shown by the blue density distribution. The darker the color the more values are present. Values from the whole three day period are shown (30.06.2015 18:00 UTC to 03.07.2015 18:00 UTC, hourly bins). The purple density distribution the skin layer experiment minus the observed LST throughout the day and the green density distribution the canopy scheme experiment minus the observed LST. 
performed for a representative period in summer 2015 with nearly clear-sky conditions (Figure 5.5) to ensure many observations. Investigations of all three implementations (see section 5.4) show that the skin layer scheme 5.3 comes closest to the observed diurnal cycle. Especially during night, the skin layer scheme outperforms the other two implementations. The skin layer scheme is used, in the remainder of this work, as it gives the best model equivalent for the LST. 


\section{Chapter 6}

\section{Synthetic Land Surface Temperature}

Observations used in an OSSE framework are generated from a nature run. Hence, a reasonable model equivalent is needed. Here, to mimic the LST retrieval provided by the Land-SAF (Chapter 4) the weighted surface temperature $T_{\mathrm{g}}$ of the COSMO-model including the skin conductivity scheme (Equation 5.19) was chosen. As this work is based on two two-day case studies, one in March 2017 and one in August 2017, two nature runs were performed (Chapter 3). Thus, from both nature runs synthetic LST observations $y^{\mathrm{t}}$ were derived. The corresponding observation operator $H$ is based on the derivation of LST by the skin conductivity scheme (Section 5.3)

$$
y^{\mathrm{t}}=H\left(x^{\mathrm{t}}\right) .
$$

The LST retrieval is available each 15 minutes. Within this 15 minutes the SEVIRI instrument scans the Earth from South to North and produces the observational data. In this thesis the synthetic LST is calculated every hour, because it is assimilated hourly. To mimic the LST derived from SEVIRI the model equivalent from the nature run $T_{\mathrm{g}}$ was interpolated to the satellite resolution of the real observations to generate the synthetic observations on the observation grid. The resolution of the satellite retrieval over Germany is around $5 \mathrm{~km}$. That means, that the spatial resolution of the nature run is about seven times higher than of the real observations. To represent the $5 \mathrm{~km}$ resolution with the nature run the nearest neighbour model grid point to the position of the observation was located. In the next step the field of the $7 \times 7$ grid points around the nearest neighbour was averaged to this point (Figure 6.1). The LST retrieval is only available over land and under clear-sky conditions, for that reasons a synthetic LST retrieval was calculated only if the simulated total cloud cover of the nearest neighbour is less than $10 \%$ and the land cover is larger than $50 \%$. The cloud cover can have a cooling/warming effect on the temperature of the Earth's surface, which lead to discrepancies between covered and clear-sky grid points. Therefore, grid points within the $7 \times 7$ grid point domain with a cloud cover larger than $10 \%$ are not used to calculate the synthetic LST retrieval. If more than $50 \%$ of the $7 \times 7$ grid box are contaminated with clouds no synthetic LST retrieval was generated at this point. 


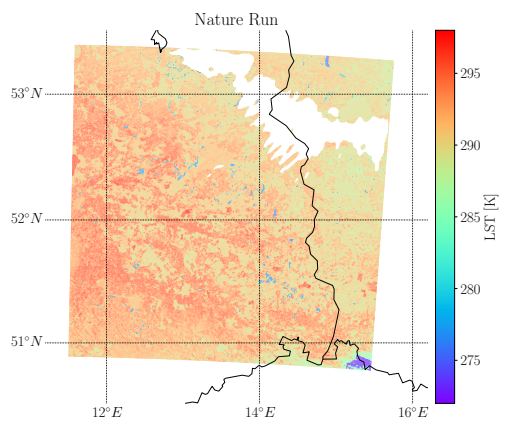

(a)

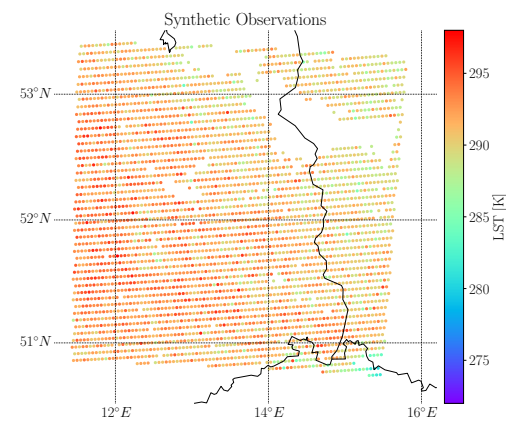

(b)

Figure 6.1: Example of synthetic LST retrieval. (a) Simulated LST of the nature run on March 27, 20171200 UTC. The white areas are clouds or outside the experimental model domain. (b) Synthetic LST derived from the nature run by interpolation. Missing observations are due to cloud cover or lakes within the model domain.

The synthetic observations generated from the nature runs $y^{\text {true }}$ are by concept perfect observations without any errors. To mimic a real-world experiment appropriate errors $\epsilon$ had to be added. This errors should combine the errors of the satellite instrument $\epsilon_{\mathrm{m}}$, the errors by the calculation of the LST retrieval $\epsilon_{\mathrm{o}}$, and the representativeness errors $\epsilon_{\mathrm{r}}$ of the LST:

$$
y=y^{\mathrm{t}}+\epsilon_{\mathrm{m}}+\epsilon_{\mathrm{o}}+\epsilon_{\mathrm{r}} .
$$

The instrumental error is due to inaccuracies of the measuring procedure, here for example sensor noise. The retrieval error is due to inaccuracies within the calculation of the LST retrieval like wrong assumptions about the water content of the atmosphere. The representativeness error includes all errors, which result from the spatial mismatch between the observed and simulated variables and the volume they depict.

An advantage of an OSSE is that the synthetic observations are by definition bias-free, because the observations generated from the truth are by construction perfect observations without any errors, i.e. no systematic errors (bias), no instrument or retrieval errors, and no representativeness errors (Masutani et al., 2010). But to obtain reliable observations for the OSSE, the characteristics of the statistical background departures of the synthetic observations, here the LST, have to be as close as possible to the characteristics of the statistical background departures of the real observations. The background departures of the real observations range between $-5 \mathrm{~K}$ to $5 \mathrm{~K}$ within our experimental period (Figure6.2, top). This error range of the statistical error should be reflected by the generated statistical synthetic observation error. To mimic the background departures of the real observations, the generated observations are perturbed by the real observation errors with values around $1 \mathrm{~K}$ to $2 \mathrm{~K}$ to (Section 5.1). Hereby, the real observation errors are multiplied by Gaussian random noise. The random noise is generated by a Gaussian random number generator (Knuth, 1998) implemented in the COSMO-model. The scaling factor of the noise is one 
and the random Gaussian shaped perturbation has a variance of one. Thus, the perturbation value of the observation is composed of the real observation error times the scaling factor times the Gaussian shaped perturbation.

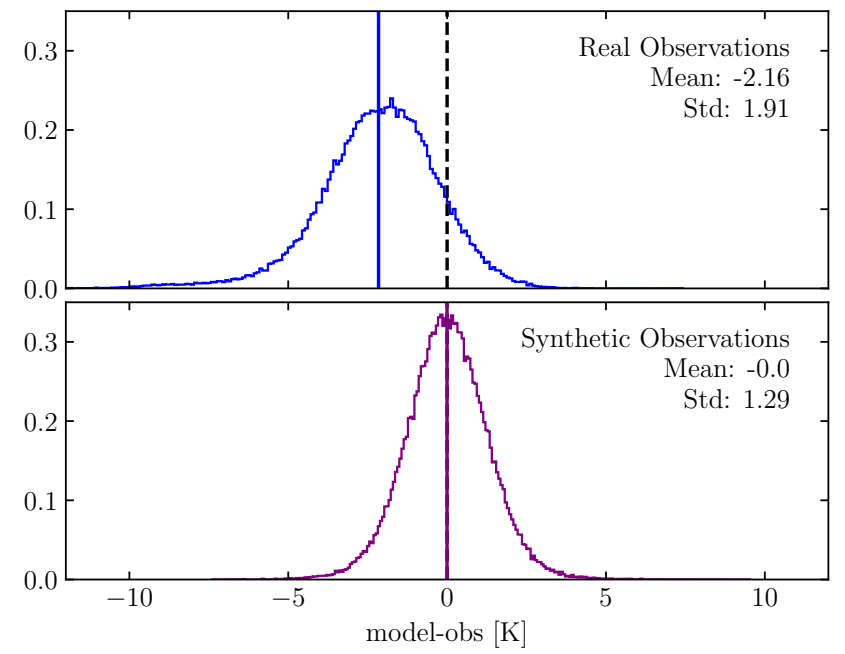

Figure 6.2: Distribution of the departure between nature run and LST observations over experiment period during March 2017. Shown are the departures from the real observations (blue) and the synthetic observations (purple).

The real observations for the two-day period are about two Kelvin warmer than the nature run, meanwhile the synthetic observations have no systematic error (Figure 6.2). The standard deviation of the synthetic observations $(1.29 \mathrm{~K})$ is smaller than the standard deviation of the real observations $(1.91 \mathrm{~K})$. Because the generated synthetic observations are bias-free and have sufficiently large statistical departures, they were used within this OSSE. Due to the smaller variance of the synthetic observations the impact of LST assimilation might be slightly overconfident. 


\section{Chapter 7}

\section{Assimilation of Land Surface Temperature}

The coupled land-atmosphere system to be investigated is described (Chapter2). The truth is generated (Chapter 3) and the synthetic LST observations have been derived from the nature runs (Chapter 6). Thus, the technical setup of the OSSEs is now complete and the coupled land-atmosphere system can be tested within this framework. First, the plausibility of the implemented coupled land-atmosphere assimilation system is reviewed in Section 7.2. Therefore, single observation experiments are carried out. Second, the direct and indirect impact of hourly LST assimilation in the coupled land-atmosphere system is analysed in Section 7.3 over two-day periods in March and August 2017.

\subsection{Preparations}

To receive ensemble perturbations on the small scale, a 'spin-up' period of two days from March 25 to March 27, 2017, for the data assimilation ensemble was carried out. During this period, real observations, i.e. measurements of radiosonde ascents and observations of air planes, $10 \mathrm{~m}$ winds, and surface pressure were assimilated to gain a more realistic difference between the nature run and the atmospheric and the soil state of the model ensemble. Thus, the assimilation cycle was initialized at March 27 at 0100 UTC. Because the soil variables, especially the deep layers, vary slowly with time the 'spin-up' period is to short to generate realistic discrepancies within the soil variables of the nature run and the assimilation ensemble. To get more realistic discrepancies between the truth and the simulated soil variables the assimilation ensemble is initialized with the icosahedral non-hydro-static model (ICON, Zängl et al., 2015) ensemble calculated over Europe. As a reminder, the nature run was initialized with the COSMO-DE model (Chapter 3). The differences between the soil model states of the COSMO-model and the ICON-model were probably still smaller than between the reality and the forecast model, but provide more useful discrepancies than between the different resolutions of the COSMO-model. The experimental model domain is a small area around Lindenberg (Chapter 3) and to avoid boundary effects, a boundary of ten grid boxes, corresponding to $28 \mathrm{~km}$, was omitted in the following evaluation. As previously mentioned, the assimilation 
cycle was based on an hourly assimilation of the synthetic LST, thus each first guess equaled a one hour forecast of the COSMO-model.

\subsection{Single Observation Experiments}

To get a first insight into the functionality of the assimilation of LST in combination with the augmented control vector, single observation experiments (SOEs) were carried out during the experimental period in March, 2017. Due to the mostly clear sky conditions during the selected periods the diurnal cycle of the synthetic LST observations as well as the diurnal cycle of the ABL are pronounced. The height of the ABL evolves with daytime, i.e. during night the ABL has stable stratification with height of several hundred meters and during daytime the mixed boundary layer can grow to several kilometers height. To evaluate the effect of LST assimilation during the diurnal cycle, the SOEs were performed at day and at night.

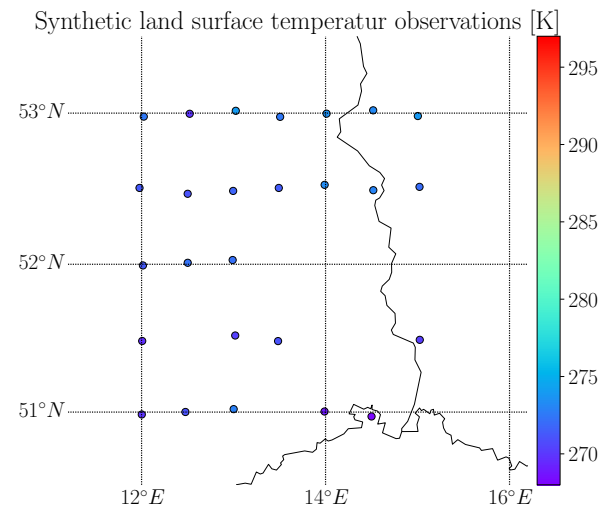

(a)

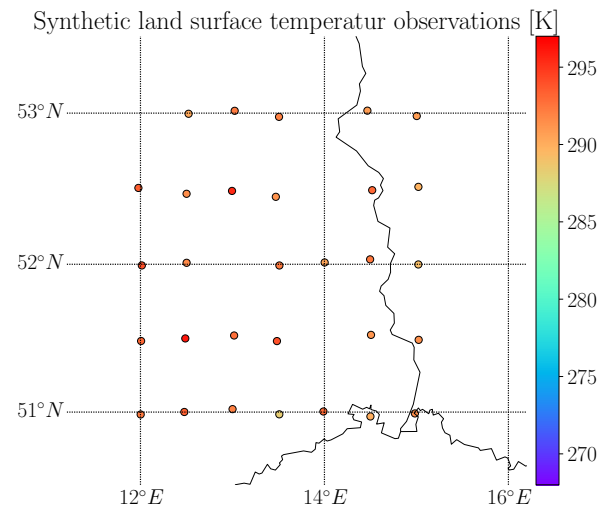

(b)

Figure 7.1: Distribution of single observations over the model domain. The nearest-neighbor of each observation has a distance of $35 \mathrm{~km}$ to $55 \mathrm{~km}$. Observation gabs in the grid of single observations are due to the cloud cover. The experiments are conducted at (a) March 27, 20170200 LT and at (b) March 27, 20171400 LT.

The single observations of synthetic LST are distributed over the experimental model domain along the longitudes and latitudes (Figure 7.1). Each $0.5^{\circ}$ in zonal and meridional direction a single synthetic LST observation is located. If clouds cover the observation location no observation is generated. The spatial distance of the observations is between $35 \mathrm{~km}$ and $55 \mathrm{~km}$. Thus, the horizontal localization scale of $5.5 \mathrm{~km}$ guarantees SOEs and the corresponding analysis grid points are only influenced by one observation each. To enhance the assimilation signal, the observation error of LST was reduced to $0.5 \mathrm{~K}$.

The impact of the assimilation of LST on the atmospheric and the soil state is limited by the horizontal and the vertical localization radius (Chapter2). The limitation of horizontal impact on the atmospheric temperature by the 


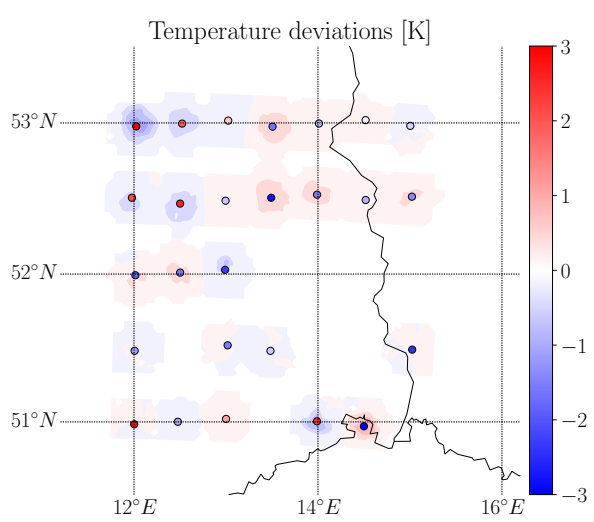

(a)

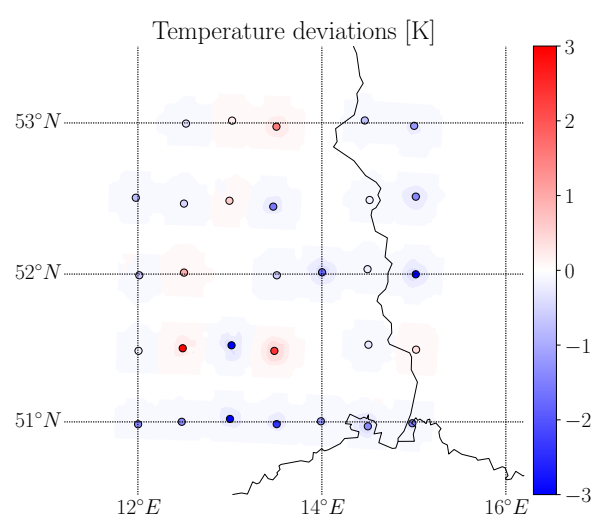

(b)

Figure 7.2: Distribution of observation minus first guess of LST combined with impact of one assimilation step on the atmospheric temperature of the lowest model level. At some SOE locations the observed synthetic LST is warmer than the simulated model equivalent (red dots) and at some locations the observation is cooler (blue dots). The impact of the assimilation step is shown by the shaded area. The atmospheric temperature of the lowest atmospheric model level is thereby raised (red) or lowered (blue). The experiments are conducted at (a) March 27, 20170200 LT and at (b) March 27, 20171400 LT.

localization is illustrated by Figure 7.2. Close to the observations, the impact of LST assimilation is clearly visible and it declines with increasing distance from the LST observations. Furthermore, Figure 7.2 illustrates in which way the observational increment of the LST influences the atmospheric temperature of the lowest model level through the analysis step. During the night (Figure 7.2a) the horizontal impact of LST assimilation is stronger than during the day (Figure $7.2 \mathrm{~b}$ ). In addition, you can see that during night a negative observational increment mostly leads to a raise of the atmospheric temperature through the analysis step while a positive observational increment causes a decrease of atmospheric temperature. The SOEs during day show a different impact. Here, a positive observational increment causes an increase of atmospheric temperature and a negative LST increment reduces the atmospheric temperature. These differences are probably caused by different background error covariances $\mathbf{P}^{\mathrm{b}}$ during the diurnal cycle. The impact of $\mathbf{P}^{\mathrm{b}}$ will be examined in more detail later in this Section and in Chapter 8 .

Figure 7.3 demonstrates the vertical expansion of the influence of LST assimilation on the atmospheric and the soil temperature. The vertical localization scale of the KENDA-system restricts the impact of the observations with height and depth. The atmospheric temperature is affected by the assimilation of LST up to a height of $2000 \mathrm{~m}$ to $3000 \mathrm{~m}$ and the soil temperature is affected down to a depth of $0.8 \mathrm{~m}$. Here, differences in the expansion of impact between day and night become apparent. The pronounced impact of LST assimilation during the night is only close to the land surface (Figure 7.3a). In most cases, the sign 


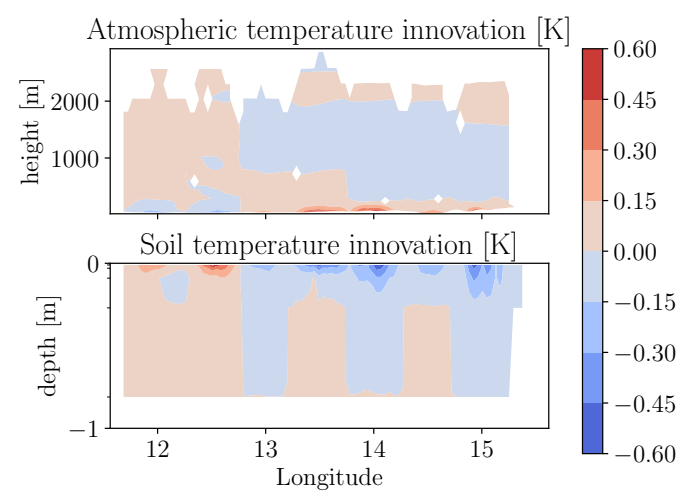

(a)

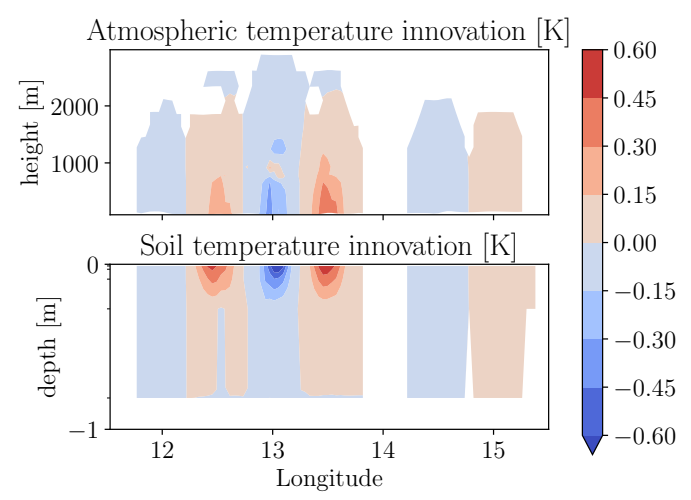

(b)

Figure 7.3: Cross sections of temperature innovations of atmosphere and soil along 52.5 latitude at 0200 LT on the night of 27 March 2017 ((a)) and along the 51.5 latitude during the day on 27 March 2017 at $1400 \mathrm{LT}((\mathbf{b}))$. The shaded area shows a raise of temperature (red) or a temperature reduction (blue).

of the lower atmospheric temperature innovation differs from the sign of the observational increment. Above the height of the ABL the sign of the atmospheric temperature innovation changes again. For example, at 52.5 latitude and 13.5 longitude the lower atmospheric temperature increases meanwhile in a height above $800 \mathrm{~m}$ it decreases. The soil temperature innovation close to the land surface is positive if the observational increment is positive and negative if the observational increment is negative. The deeper soil layers change the sign of the temperature observation partially. Again, at 52.5 latitude and 13.5 longitude the soil temperature close to the surface is reduced, but the deeper soil level temperature is raised. During the day the pronounced impact of LST assimilation reaches further in the atmosphere and deeper into the soil than during nighttime (Figure 7.3b). This fits well with the diurnal development of the atmospheric boundary layer height. The stable boundary layer during night has only a height of about $300 \mathrm{~m}$ whereas the mixed boundary layer during day raises up to $2000 \mathrm{~m}$ and higher.

To get further insight into the process of LST assimilation, atmospheric and soil temperature profiles, and correlation profiles are evaluated in the following Sections. The correlation profiles illustrate how the background error covariance matrix $\mathbf{P}^{\mathrm{b}}$ passes on the impact of LST assimilation on the atmospheric and the soil temperature at day (Section 7.2.1) and at night (Section7.2.2).

\subsubsection{Day: 27 March, 20171400 LT}

The SOE at $52.0^{\circ}, 014.0^{\circ}$ was chosen as an example to illustrate how the augmented COSMO-KENDA system works during daytime (Figure 7.4). At $1400 \mathrm{LT}$ the land surface is around $5 \mathrm{~K}$ warmer than the atmospheric temperature due to solar radiation. Hereby, the synthetic LST observation and the lower atmospheric temperature of the nature run are cooler than the first guess (Figure 7.4a, left panel). Within the mixed boundary layer the atmospheric 


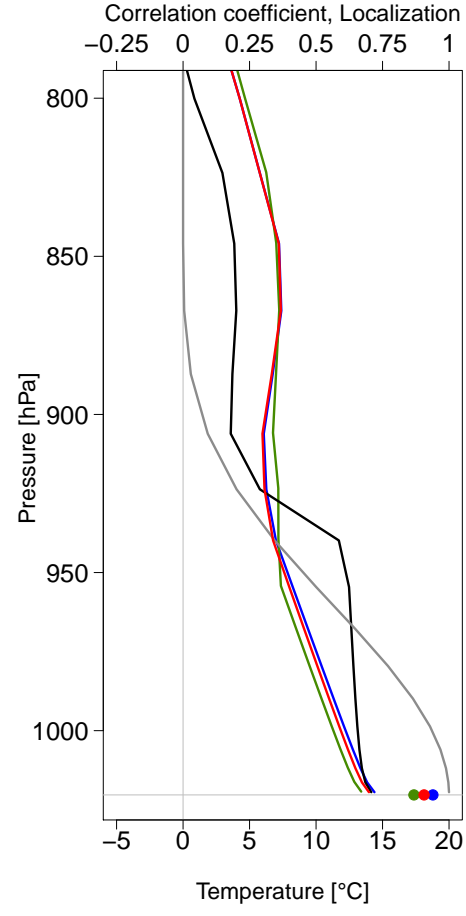

(a)

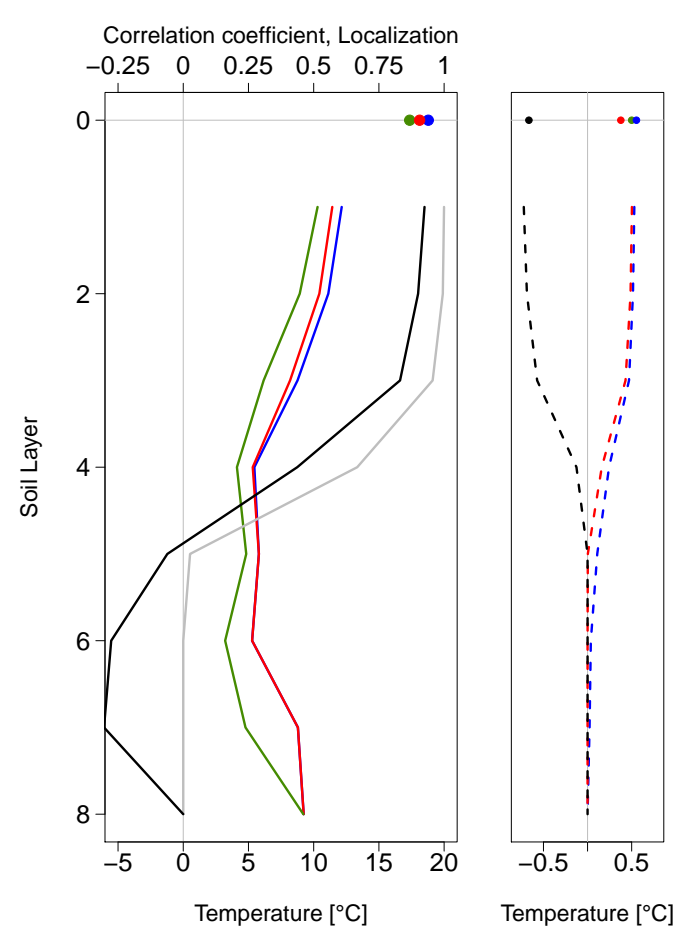

(b)

Figure 7.4: (a) Impact of LST assimilation at March 27, 2017, 1400 LT. Left panel: atmospheric temperature profiles of first guess (blue), analysis (red) and, truth (green), localization function (grey), and the correlation between LST and atmospheric temperature (black). The big dots indicate LST first guess (blue), LST analysis (red) and LST observation (green). Right panel: atmospheric temperature spread profiles of first guess (blue, dashed) and analysis (red, dashed) and, of the atmospheric temperature increment (black, dashed). The small dots indicate the spreads of LST first guess (blue) and LST analysis (red), the LST observation error (green) and, the LST increment (black). (b) Impact of LST assimilation on the soil temperature. Location of SOE: latitude: $52.0^{\circ}$, longitude: $014.0^{\circ}$. Figure taken from Sgoff et al. (2020).

temperature of the first guess and of the nature run decrease the same way with altitude. However, the mixed boundary layer of the first guess has a higher expansion than the mixed layer of the truth. Within the ABL of the first guess the correlation between the LST and the atmospheric temperature is positive. More precisely, the correlation coefficient is about 0.6 and is constant over the height of the ABL. Within the ABL the correlation coefficients of the SOEs are similar, but above the mixed boundary layer the SOEs show different correlations between the LST and the atmospheric temperature. The observation error of $0.5 \mathrm{~K}$ is slightly smaller than the spread of the model equivalent of the first guess (Figure 7.4a, right panel). The analysis step pulls the land surface temperature in direction of the observation. The resulting land surface temperature of the analysis is lower than the first guess. Because of the positive correlation between the LST and the atmospheric temperature, the atmospheric 
temperature is also lowered, which results in a constant, negative increment over the whole mixed boundary layer. The vertical localization restricts the vertical impact of the assimilation of LST, therefore the atmospheric temperature increment decreases with height as well as the localization function. Due to the temperature reduction of the analysis step the temperature of the ABL is closer to the nature run than the first guess, i.e. the assimilation of LST is able to improve the lower atmospheric temperature.

As the atmospheric temperature, the soil temperature is cooler than the land surface around noon. Within the upper four soil levels the soil temperature of first guess and nature run decreases with depth (Figure $7.4 \mathrm{~b}$, left panel). The temperature profile of the nature run is similar to the temperature profile of the first guess temperature, but it is lower over all soil layers. Only exception is the climate layer, where the soil temperature of both, by definition, is equal. The temperature of the upper soil levels, down to $1 \mathrm{~m}$ depth, are positively correlated with the land surface temperature. Meanwhile, the upper three levels have a correlation coefficient over 0.75 , the positive correlation decreases within the next levels close to zero. As the example, all conducted SOEs show the positive correlation between LST and soil temperature and a similar decrease of the correlation coefficient with depth (not shown). But the correlation coefficients of the soil layers below $1 \mathrm{~m}$ are no longer uniform across the SOEs. Thus, as in the atmosphere, the analysis step leads to a negative temperature increment (Figure 7.4b, right panel). Due to the implemented soil localization the impact of LST assimilation is restricted to the upper five layers and approaches zero within the layers, which are not affected by the diurnal temperature cycle. The reduction of soil temperature pulls the analysis closer to the nature run and improves the soil temperature of the upper soil levels.

\subsubsection{Night: 27 March, 20170200 LT}

The SOE at $53.0^{\circ}, 012.5^{\circ}$ was chosen to illustrate exemplary how the assimilation of LST influences the ABL and the soil temperature during night. In contrast to the mixed boundary layer during day the stable stratified boundary layer during night has only a height of about $0.5 \mathrm{~km}$. The temperature inversion typical for the stable boundary layer appears in the first guess and the nature run (Figure 7.5a, left panel). Within the stable boundary layer the atmospheric temperature increases with height and above the top of the ground inversion at about $0.5 \mathrm{~km}$ it starts to decrease. The temperature difference between land surface and lower atmosphere is up to $10 \mathrm{~K}$, i.e. the LST is noticeable cooler than the atmosphere above. This temperature difference is even more significant in the first guess than in the nature run. Within the ABL the correlation between the LST and the atmospheric temperature determined by the first guess ensemble is negative and more pronounced close to the land surface. The anti-correlation between LST and atmospheric temperature is the dominant behavior of the conducted SOEs (not shown), but the correlations between the land surface temperature and the atmospheric temperature of the SOEs differ. The most SOEs are anti-correlated, some are correlated, some are not correlated at all. In some cases the correlations of the first guess do not match with the 
truth and lead to wrong increments. Thus, the system is working as intended, but its wrong ensemble correlations result in wrong increments. The observation error of LST of $0.5 \mathrm{~K}$ is clearly smaller than the spread of the LST model equivalent with its maximum of about $3 \mathrm{~K}$ near the surface (Figure $7.5 \mathrm{a}$, right panel). The analysis of the LST is warmer than the first guess of the LST and thus closer to the observed truth. Due to the negative correlation between the LST and the atmospheric temperature, the analysis step reduces the atmospheric temperature and pulls it closer to the nature run. The vertical localization scale during night equals the localization scale of the day (Figure 7.4, left panel). The negative increment values are highest near the surface and decrease within the stable boundary layer close to zero.

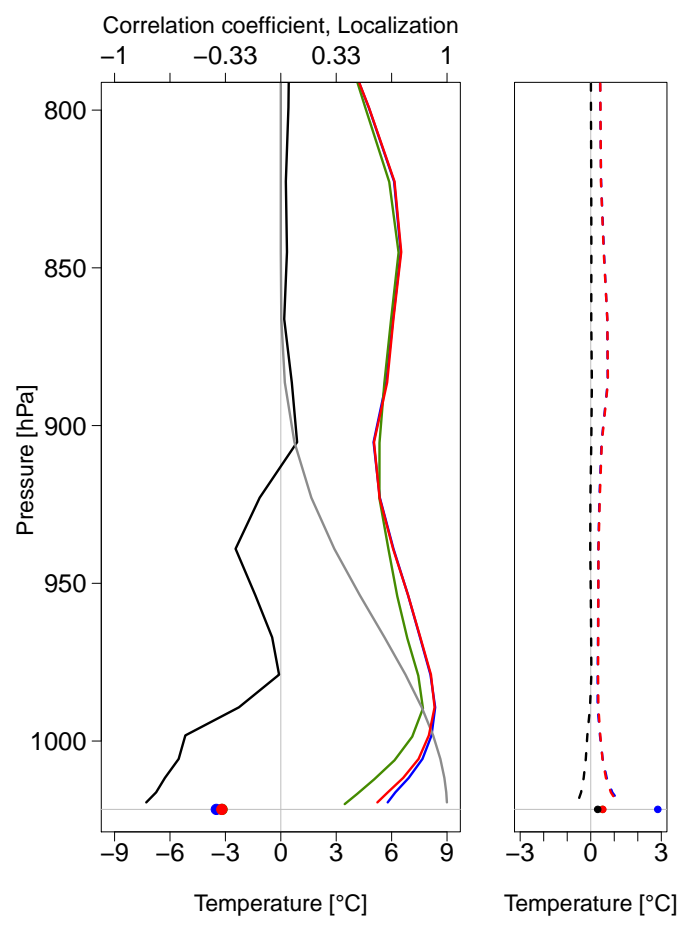

(a)

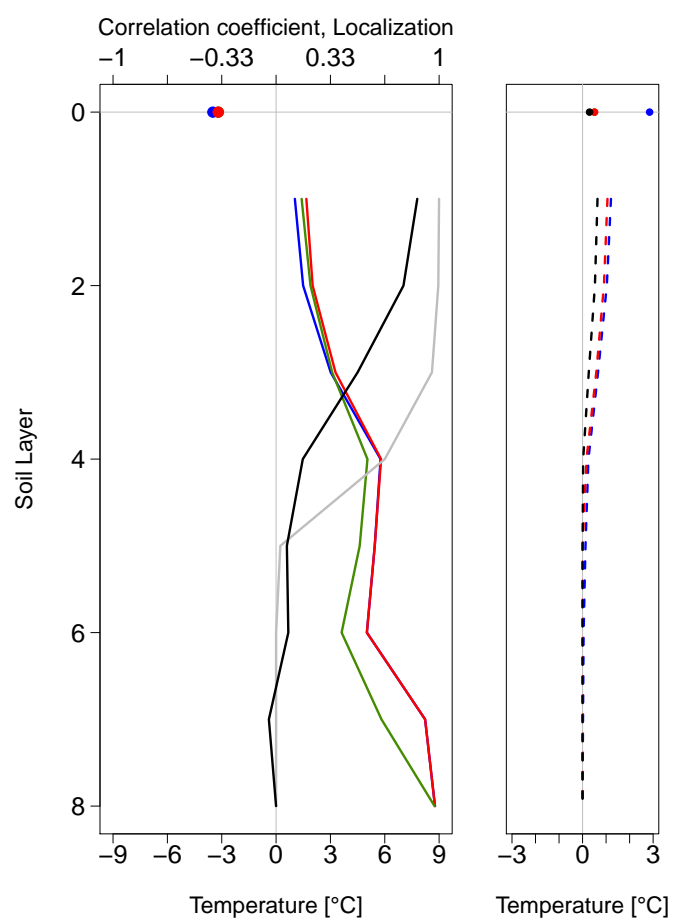

(b)

Figure 7.5: As for Figure 7.4, but at March 27, 2017, $0200 \mathrm{~h}$. The synthetic observation of LST (green dot) is located behind the model equivalent LST of the analysis (red dot) and thus not visible. Same applies to the LST observation error (small green dot), which is located behind the spread of analysis LST (small red dot). Location of SOE: latitude: $53.0^{\circ}$, longitude: $012.5^{\circ}$. Figure taken from Sgoff et al. (2020).

In comparison with daytime, the soil temperature profile is different during nighttime because during night the upper soil levels are cooler than the deeper soil levels (Figure $7.5 \mathrm{~b}$, left panel). In the first three levels the first guess soil temperature is warmer than the true soil temperature. In the soil layers below it is the other way around. As at noon, the correlation between LST and the upper soil levels is positive. This also applies to the remaining SOEs (not shown). As during daytime the soil temperature increment is positive and 
declines with depth as given by the localization function (Figure 7.5b, right panel). The assimilation of LST pulls the analysis to warmer temperatures, but slightly to strong. Thus, the analysis is closer to the truth, but slightly warmer.

To sum up, at first glance the augmented COSMO-KENDA-system works as expected. Because the simulated LST is a diagnostic model variable it is not part of the control vector. Hence, the impact of the assimilation of LST is dominated by covariances between the LST and the prognostic variables like the atmospheric or the soil temperature. As presented above, the LST and the near surface temperatures can be correlated, anti-correlated or not correlated at all and depending on the background covariances $\mathbf{P}^{\mathrm{b}}$ the temperature innovations can be positive or negative. More precisely, if LST and the temperature are correlated a positive observation increment yields to an increase in atmospheric temperature. But if LST and the temperature are anti-correlated the positive observation increment yields to a decrease of the temperature. This shows impressively how important accurate model error covariances $\mathbf{P}^{\mathrm{b}}$ are.

\subsection{Augmented Control Vector Tests}

After the discussion of the previous Section 7.2 about the functioning of the coupled assimilation system, the impact of LST assimilation is evaluated in the following. For this purpose, different configurations of the control vector were investigated to clarify the question: How good is the augmented control vector compared to a control vector which contains only one part of the coupled system, atmosphere or soil. Two two-day experimental periods were defined: one early spring period (March 27, 20170000 UTC to March 29, 20170000 UTC) and one summer period (August 28, 20171200 UTC to August 30, 20171200 UTC).

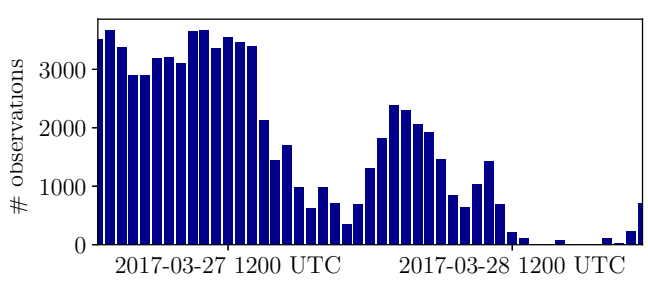

(a)

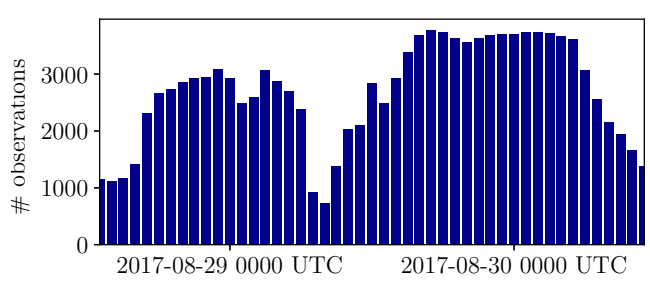

(b)

Figure 7.6: (a) Number of active LST observations during the twoday period of March 27 to March 29, 2017 and (b) of August 28 to August 30, 2017. The number of observations is limited by the cloud cover of the nature run. Figure taken from Sgoff et al. (2020)

The early spring experiment started with clear sky conditions on the first day but got cloudier on the second day (Figure 7.6a), thus on the first day much more LST observations were possible and could be used within the assimilation cycle. The summer experiment was mainly cloud free on both days. But clouds came up during the morning hours, reducing the number of observations over 
the morning hours and midday.

To evaluate the impact of LST assimilation within the fully coupled COSMOKENDA-system one reference experiment, named Control in the following, and three experiments with different control vector configurations were set up. Control is a so-called open loop experiment, i.e. the experiment was run within the hourly assimilation cycle, but omitting the analysis step. Thus, each first guess of Control is a one hour forecast, started from its former first guess.

Table 7.1: Experiments with different control vector settings. Table from Sgoff et al. (2020)

\begin{tabular}{|l|l|}
\hline Experiment Name & Control Vector Setup \\
\hline \hline Control & no Assimilation \\
\hline EXPatmos & Atmosphere \\
\hline EXPsoil & Soil \\
\hline EXPatso & Atmosphere and Soil \\
\hline
\end{tabular}

The three experiments assimilating synthetic LST are distinguished by different versions of the control vector (Table 7.1). The control vector of experiment EXPatmos included only the atmospheric variables, this corresponds to the current COSMO-KENDA setting. Thus the analysis step had a direct influence on the atmospheric variables. However, the soil was only indirectly influenced by the interaction with the changed atmosphere. In contrast, EXPsoil had a control vector, which only included the soil variables. Here, the analysis step had a direct influence on the soil, but only an indirect effect on the atmospheric variables. The third experiment, EXPatso, was the fully coupled assimilation system. The control vector of EXPatso included the atmospheric and the soil variables and the analysis step had a direct impact on atmosphere and soil simultaneously.

Even tough the LST was not part of the control vector, it was still possible to calculate the analysis of LST. By the assimilation of LST the deviations between the observed LST and the simulated LST should decrease by definition. Figure 7.7 illustrates that this was the case. The deviations between observed and simulated LST of EXPatso were reduced during the March and during the August period. In both experimental periods the mean of the analysis deviations is closer to zero than the mean of the first guess deviations. Furthermore, in both cases the standard deviation of the observation minus analysis is smaller than of the observation minus first guess. However, the analysis of the LST was discarded, as it is a diagnostic variable. Accordingly, the lasting impact of the assimilation of LST on atmosphere and soil was caused by the background error covariances $\mathbf{P}^{\mathrm{b}}$, which transfer the information of the LST observation to the model.

The conducted experiments were evaluated against the nature run, which is assumed to be the true state of the atmosphere and soil. This assumption is an advantage of OSSEs because the whole atmospheric and soil system can be 


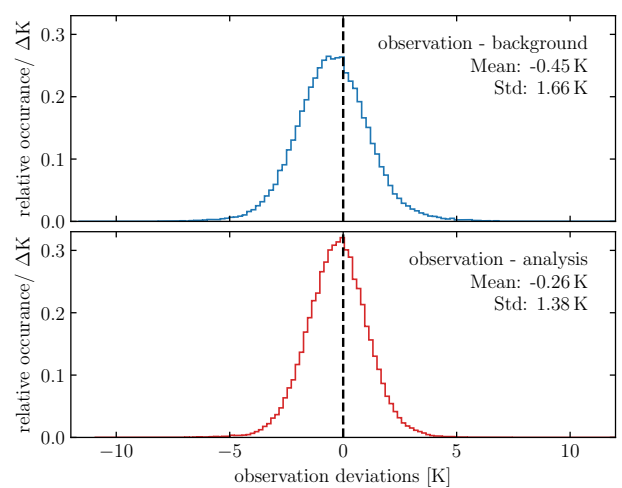

(a)

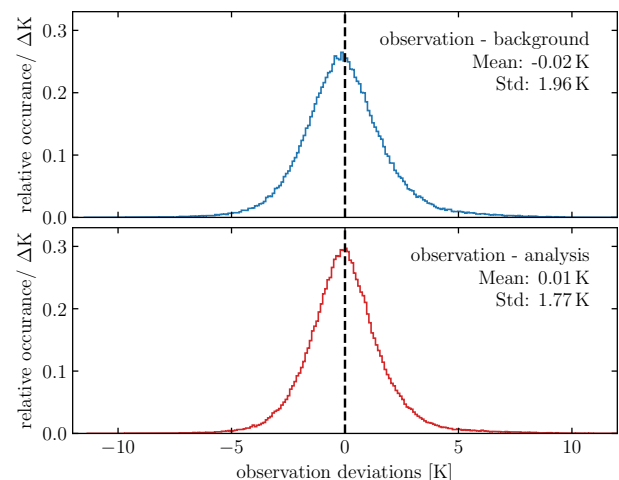

(b)

Figure 7.7: Distribution of the departure between LST observations and COSMO-model over the experimental period during March 2017 (a) and during August 2017 (b). Shown are the departures from the background/first guess (blue) and the analysis (red) of EXPatso.

evaluated. Under real conditions this would only be possible to a limited extent, as verification can only be carried out against further observations, which were limited in space and time. How well the results of the experiments can be transferred to the real conditions depends on how well the nature run and the synthetic LST observations reflects the real system in its properties. This OSSE is a first step in the direction of a fully coupled land-atmosphere assimilation system. Because the synthetic LST provides information for atmosphere and soil as well it is a useful observation to evaluate the coupled system.

\subsubsection{Mean Error Evaluation}

Firstly, the spatial mean error (ME)

$$
M E=\frac{1}{N} \sum_{i=1}^{N}\left[x(i)-x_{\text {true }}(i)\right]
$$

was calculated to determine systematic deficiencies of simulated near surface temperature and humidity variables. Here $N$ is the number of grid points of the domain without the boundary regions, $x$ the simulated value of the respective experiment and $x_{\text {true }}$ the truth value. All model grid points were considered regardless of the availability of observations.

The temporal evolution of the spatial ME of the atmospheric temperature of the two-day early spring case is illustrated by Figure 7.8a. Compared to the nature run, the control run and the experiments have a too warm atmospheric temperature within the ABL (Figure 7.8a). Furthermore, the ME of the control run and the experiments has a diurnal cycle within the ABL, i.e. during night and the morning hours the ME is larger than after midday and in the evening. Above the ABL, the ME is negative with values lower than $0.5 \mathrm{~K}$ for each experiment. The positive ME of the Control has values up to $1.5 \mathrm{~K}$. The three 


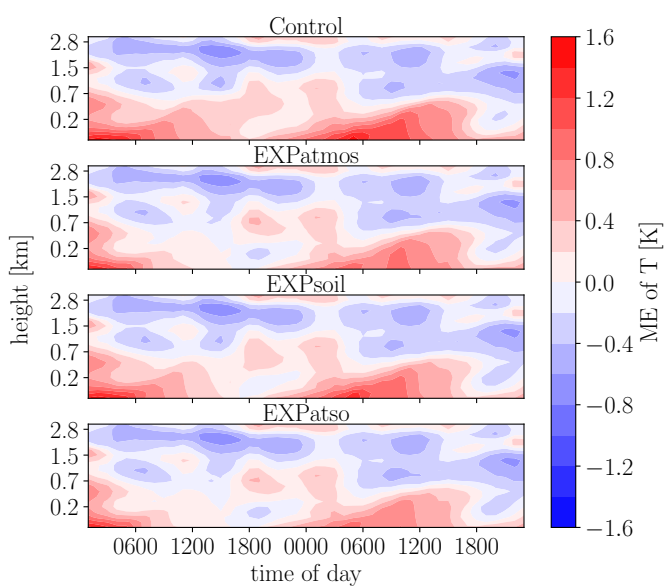

(a)

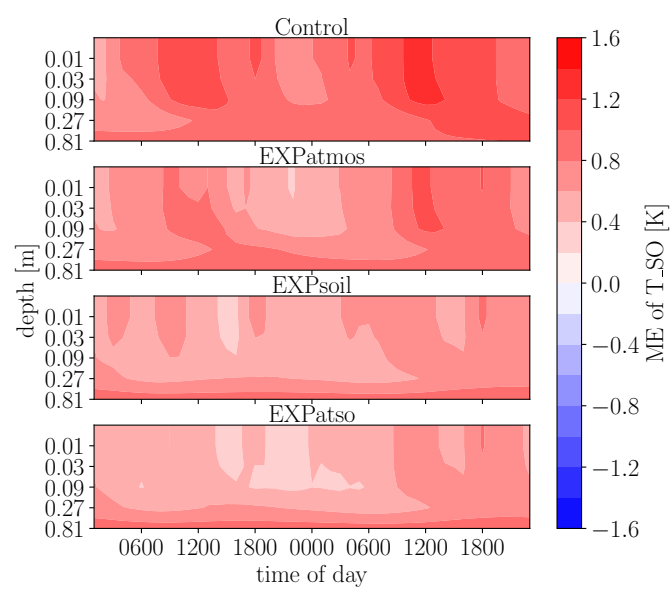

(b)

Figure 7.8: Mean error evolution (a) of the temperature $(T)$ of the ABL and (b) of the temperature of the soil levels $\left(T_{\mathrm{so}}\right)$ from March 27 to March 29, 2017. First guess evaluated against truth averaged over the whole experiment domain. Shown are the mean error of the Control (first panel), of EXPatmos (second panel), of EXPsoil (third panel) and of EXPatso (fourth panel). Red shaded areas indicate a warmer model state and blue shaded areas indicate a cooler model state. Figure taken from Sgoff et al. (2020)

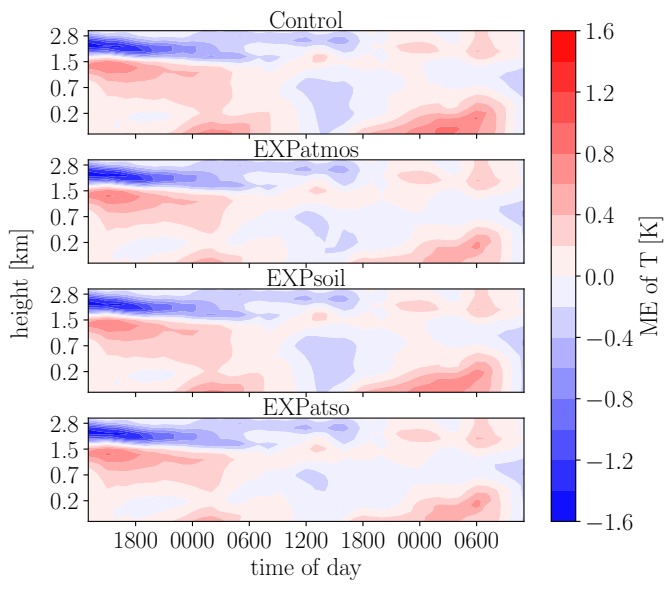

(a)

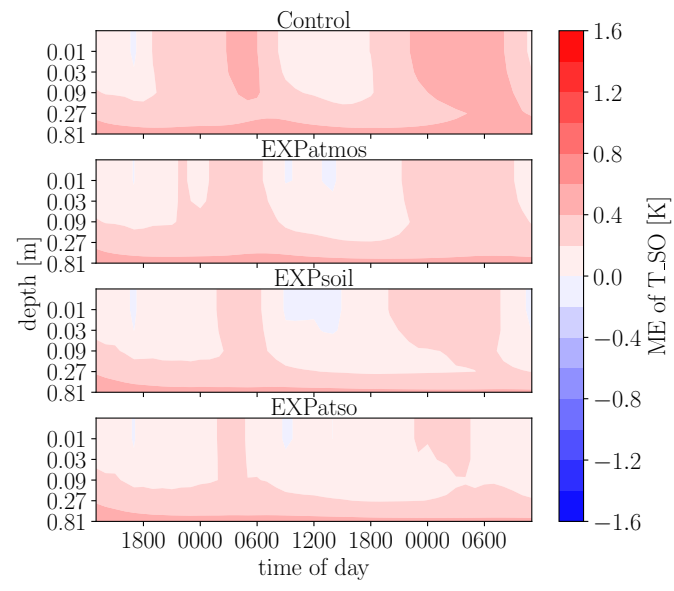

(b)

Figure 7.9: As Figure 7.8. Figure taken from Sgoff et al. (2020)

experiments behave similar to the Control, however with a reduced ME within the ABL. Especially EXPatmos and EXPatso have a reduction of spatial ME up to $0.6 \mathrm{~K}$ in the morning hours. Above $0.7 \mathrm{~km}$ the influence of LST assimilation decreases and the negative ME remains nearly unaffected.

The soil temperature has a consistent positive ME over the upper soil layers and a diurnal cycle (Figure 7.8b). The diurnal cycle of soil temperature ME has a six hours shift compared to the diurnal cycle of the ABL temperature $\mathrm{ME}$, namely the ME is highest around midday and lowest around midnight. 
The Control shows most clearly this behavior. Each of the three early spring experiments has a reduced ME compared to Control. The ME reduction is most pronounced in EXPatso with a reduction of the mean error of up to $0.7 \mathrm{~K}$ around midday.

The temporal evolution of the spatial ME of the atmospheric temperature of the two-day summer case (Figure 7.9a) has a similar course as the early spring experiments. The atmospheric temperature of the Control and the experiments is too warm and the $\mathrm{ME}$ of the atmospheric temperature has also a diurnal cycle with a maximum ME between 0000 UTC and 0600 UTC. The ME within the $\mathrm{ABL}$ of the summer experiments is on average $0.4 \mathrm{~K}$ smaller than the ME of the spring experiments, i.e. within the ABL the temperature of the control run and the experiments is closer to the atmospheric temperature of the nature run than in March. From August 281300 UTC to August 290100 UTC a negative $\mathrm{ME}$ is visible between $1.5 \mathrm{~km}$ and $2.8 \mathrm{~km}$ height in each experiment, but as in the early spring case the atmospheric temperature above the ABL is nearly unaffected by the assimilation of LST.

The temperature of the upper soil layers of the summer case (Figure 7.9b) has a consistent positive ME which is on average $0.6 \mathrm{~K}$ smaller than the ME of the early spring case. The ME is highest between 0000 UTC and 0600 UTC. A reduction of about $0.2 \mathrm{~K}$ of $\mathrm{ME}$ is visible in all three experiments compared to the ME of the control run. In comparison with the truth the atmospheric

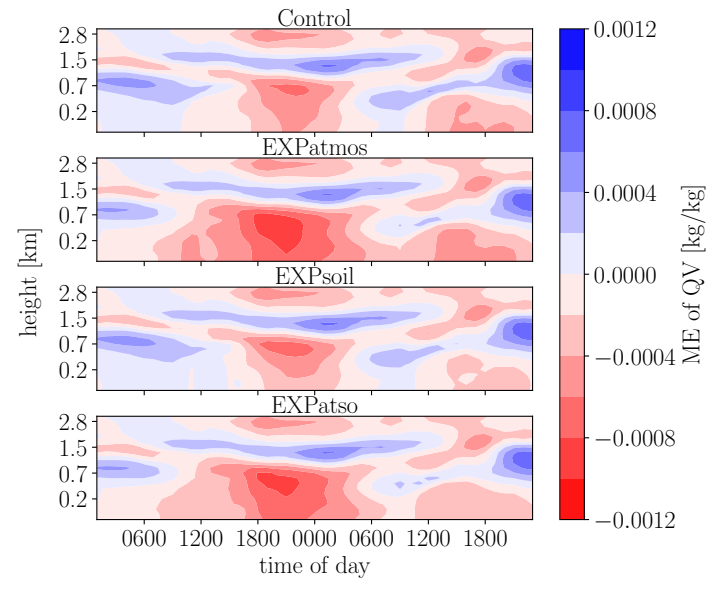

(a)

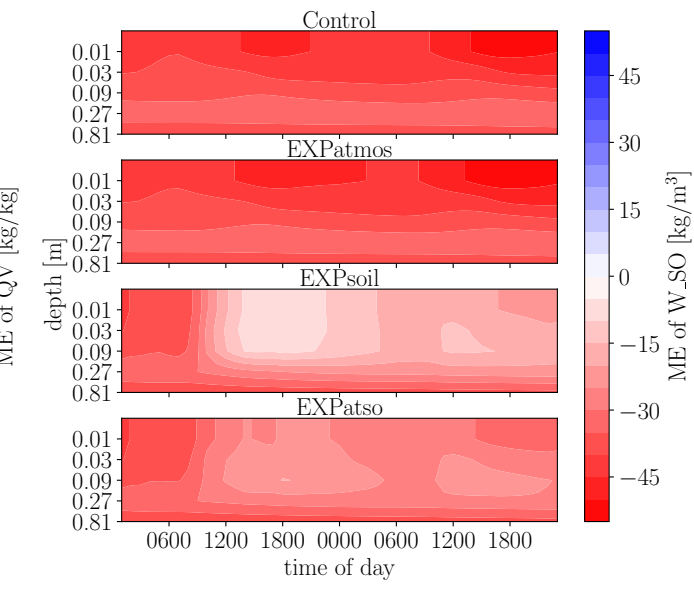

(b)

Figure 7.10: Like Figure 7.8 but here the evolution of (a) the specific humidity $(Q V)$ of the ABL and of $(\mathbf{b})$ the soil moisture $\left(W_{\mathrm{so}}\right)$ of the soil levels is shown. Red shaded areas indicate a drier model state and blue shaded areas indicate a more humid model state.

specific humidity is reduced in the Control and the three control vector experiments. It is important to note that the assimilation of LST causes a further reduction of atmospheric moisture (Figure 7.10b), if the control vector includes atmospheric variables. Especially during the night of the 27 to 28 of March the specific humidity decreases. The nightly decrease of the specific humidity is about $10 \%$. Above the ABL the atmosphere of the experiments is more humid 
than the nature run. As already demonstrated for the temperature also the specific humidity above $0.7 \mathrm{~km}$ is nearly unaffected.

All upper model soil levels of the assimilation experiments start with $10 \%$ to $20 \%$ less humidity per cubic metre than the nature run. In the Control and the EXPatmos this does not change during the two-day experiment period, the closer to the land surface the more moisture is missing (Figure 7.10b). In EXPsoil the assimilation of LST introduces humidity directly into the soil through the soil variables included in the control vector. The ME of soil moisture reduces to less than $10 \mathrm{~kg} / \mathrm{m}^{3}$ within the soil levels close to the land surface. This improvement is mostly retained for the remaining experiment period. This effect also occurs in a weakened form in EXPatso.

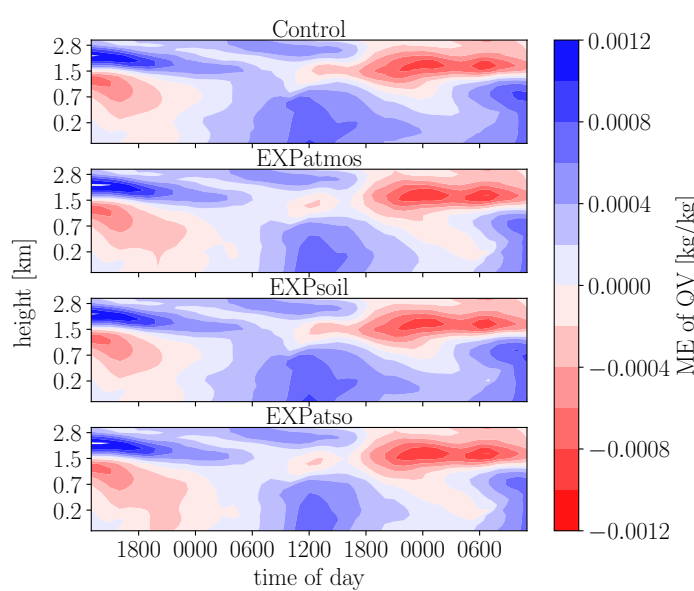

(a)

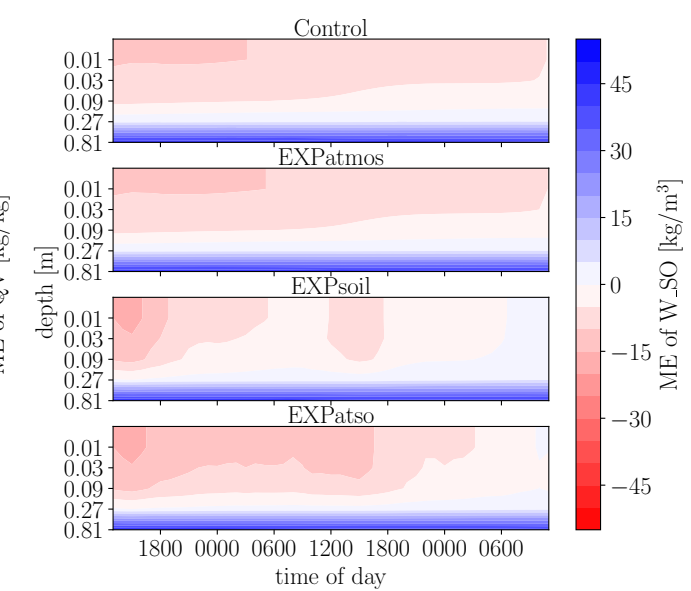

(b)

Figure 7.11: As Figure 7.10 but for the summer case.

In contrast to the early spring case, the specific humidity of the summer case is higher in comparison with the truth (Figure 7.11a). Especially around noon the reference and the three experiments are more humid than the true state of the atmosphere. The assimilation of LST causes as in the early spring case a reduction of the specific humidity of the $\mathrm{ABL}$, if the control vector includes the atmospheric variables. In particular during the nights the specific humidity decreases (EXPatmos and EXPatso). Above the ABL in the first 24 hours, the atmosphere of the experiments is more humid than the nature run, while in the following 24 hours the atmosphere above the ABL is drier. But due to the vertical localisation the assimilation of LST hardly affects the atmosphere above the ABL at all.

The upper soil levels are drier than the nature run but the difference between nature run and experiments in August is smaller than in March (Figure 7.10b and $7.11 \mathrm{~b}$ ). The levels below $0.5 \mathrm{~m}$ depth, on the other hand, are much wetter than the truth and not affected by the assimilation of LST. The impact of LST assimilation on the soil moisture is only visible if the soil variables are part of the control vector (EXPsoil and EXPatso). In EXPsoil the soil becomes drier in the first six hours, afterwards moisture is introduced so that the difference to 
the truth decreases and the ME is less than $5 \mathrm{~kg} / \mathrm{m}^{3}$ (Figure 7.11b, third panel). In EXPatso the phase in which moisture is lost lasts over 24 hours. Hence, the ME of soil moisture is greater than in the other experiments (Figure 7.11b, fourth panel). From the evening of August 29, moisture is introduced into the soil and lowers the ME to below $5 \mathrm{~kg} / \mathrm{m}^{3}$.

\subsubsection{Root-Mean-Square Error Evaluation}

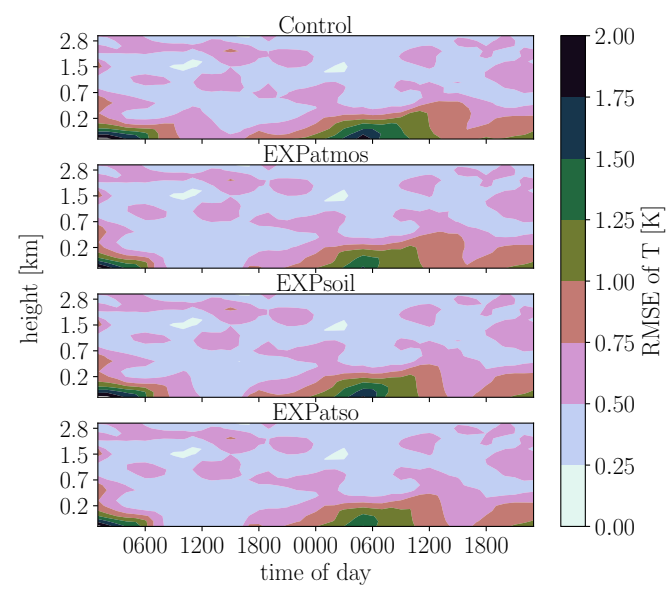

(a)

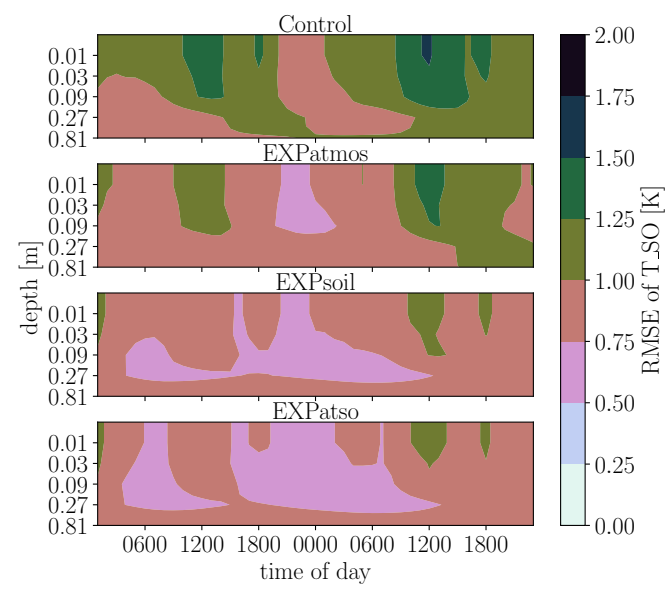

(b)

Figure 7.12: RMSE evolution of (a) temperature of atmospheric boundary layer $(T)$ and of (b) soil levels $\left(T_{\mathrm{so}}\right)$ from March 27 to March 29, 2017. First guess evaluated against truth averaged over the whole experiment domain. Shown are the RMSE of Control (first panel), of EXPatmos (second panel), of EXPsoil (third panel) and of EXPatso (fourth panel). Darker colors indicate larger RMSE. Figure taken from Sgoff et al. (2020).

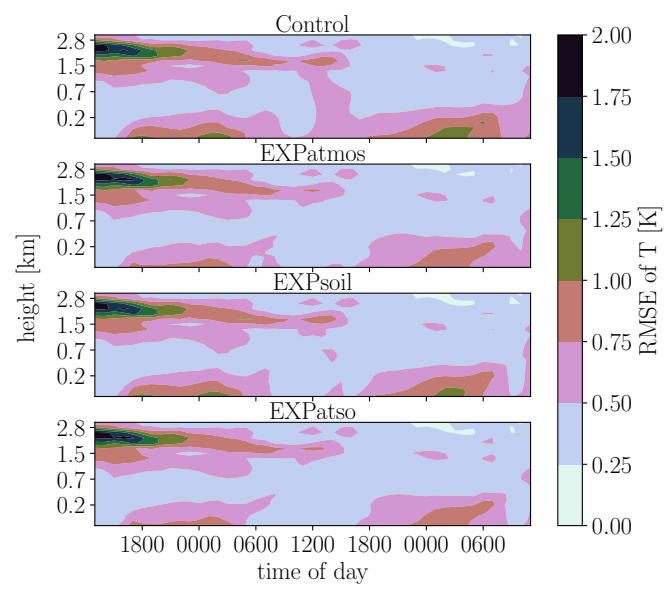

(a)

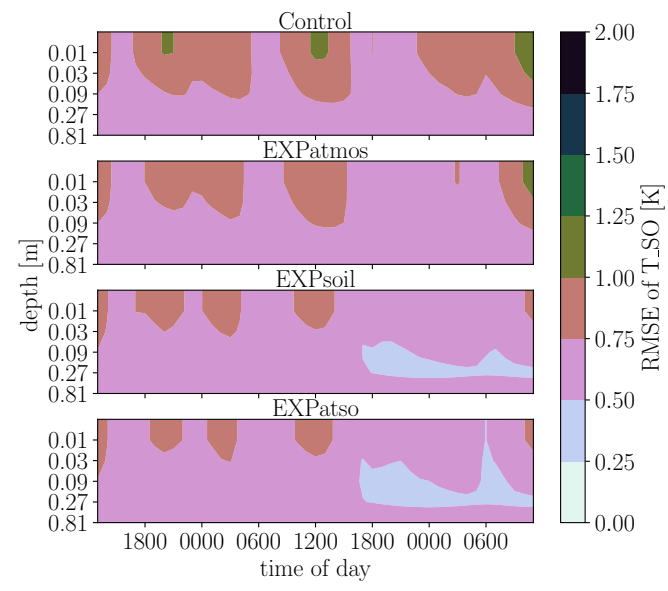

(b)

Figure 7.13: As Figure 7.12 but from August 28 to August 30, 2017.

Figure taken from Sgoff et al. (2020). 
Next the root-mean-square error (RMSE)

$$
R M S E=\sqrt{\frac{1}{N} \sum_{i=1}^{N}\left[x(i)-x_{\text {true }}(i)\right]^{2}}
$$

is examined for the LST related variables. During the March and the August case study, the RMSE of the boundary layer temperature has a diurnal cycle, similar as for the ME (Figure 7.12a and 7.13a). The maximum RMSE within the ABL during the spring and the summer experiments is close to the surface in the lowest $0.1 \mathrm{~km}$ between 0000 UTC and 0600 UTC. During the August experiment another maximum of RMSE is located at the same height as the negative ME in August (Figure 7.9a). The RMSE of atmospheric temperature is reduced in each assimilation experiment of both experimental periods.

In the early spring and the summer period, the RMSE of the soil temperature is higher than the RMSE of the boundary layer temperature (Figure $7.12 \mathrm{~b}$ and $7.13 \mathrm{~b}$ ). The assimilation of LST reduces the RMSE of each influenced soil level during the summer and spring period, especially if the soil variables are included in the control vector (EXPsoil and EXPatso). In comparison with the early spring case, the summer case soil and boundary layer temperature have a smaller RMSE (Figure 7.12 and Figure 7.13).

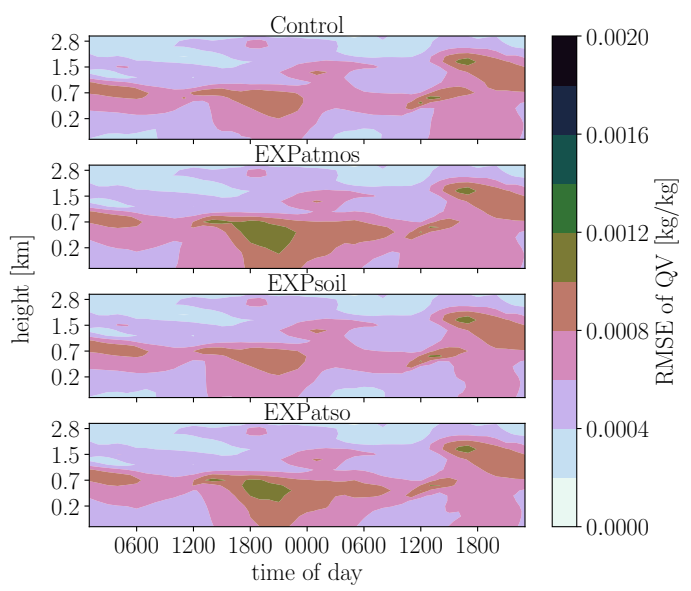

(a)

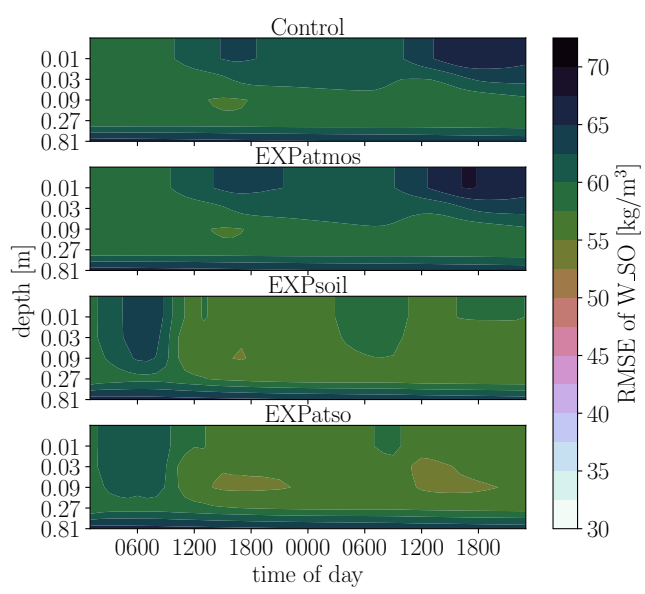

(b)

Figure 7.14: Like Figure 7.12 but with RMSE evolution of (a) specific humidity $(Q V)$ of atmospheric boundary layer and (b) of soil moisture $\left(W_{\text {so }}\right)$.

The RMSE of specific humidity during the March period has a diurnal cycle with the maximum RMSE between 1800 UTC and 0000 UTC around $0.7 \mathrm{~km}$ height (Figure 7.14a). The RMSE for the specific humidity is larger if the atmospheric humidity variables are included into the control vector (Figure 7.14a, second and fourth panel).

The RMSE of the soil moisture of Control and EXPatmos is very similar. Both start with a RMSE of about $55 \mathrm{~kg} / \mathrm{m}^{3}$ which grows after twelve hours of 
assimilation cycle up to over $65 \mathrm{~kg} / \mathrm{m}^{3}$ within the upper soil levels (Figure $7.14 \mathrm{~b}$, first and second panel). If the soil variables are included in the control vector the assimilation of LST has more pronounced impact on the soil moisture (Figure 7.14b, third and fourth panel). Within the first twelve hours of the assimilation experiments the RMSE of the soil moisture grows up to $65 \mathrm{~kg} / \mathrm{m}^{3}$, but then the RMSE is reduced to values lower than $55 \mathrm{~kg} / \mathrm{m}^{3}$.

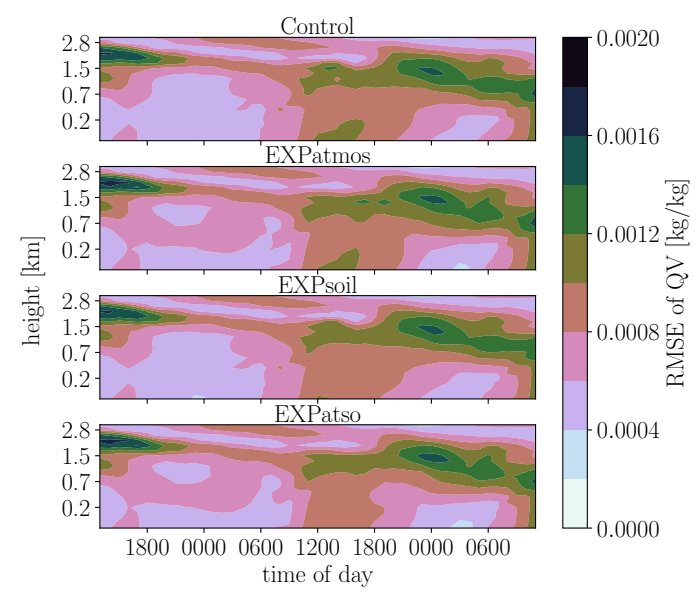

(a)

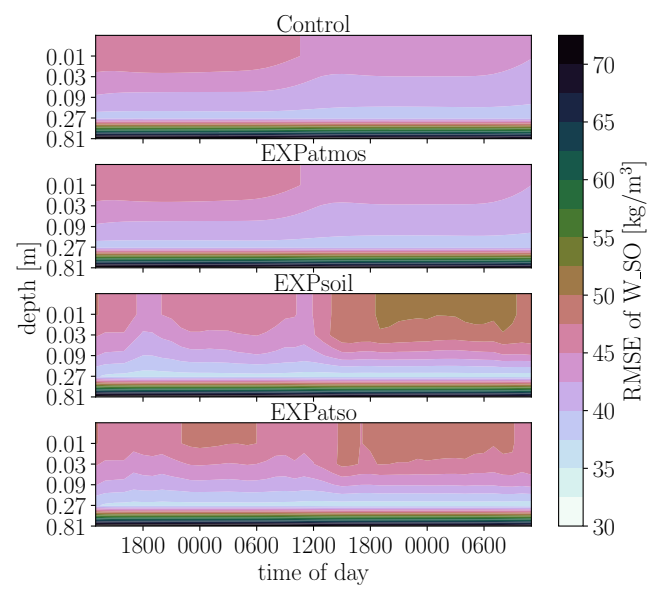

(b)

Figure 7.15: Like Figure 7.14 but from August 28 to August 30, 2017.

The RMSE of specific humidity during the August period shows a diurnal cycle as well (Figure 7.15). Its maximum close to the surface is between 1200 UTC and 1800 UTC and its minimum is between 0000 UTC and 0600 UTC similar to the diurnal cycle of the ME of specific humidity during the summer case study. The highest RMSE values of the August period are found around the top of the ABL.

On average the RMSE of soil moisture of the upper soil levels during August is smaller than during March. Only the deeper layers have a RMSE higher than $55 \mathrm{~kg} / \mathrm{m}^{3}$ (Figure 7.15b). In the deeper layers the nature run is wetter than the assimilation experiments. This clear difference is also reflected in the RMSE of soil moisture. While the RMSE of soil moisture decreases in the upper layers as long as the soil variables are not part of the control vector (Figure 7.15b, first and second panel), it increases in the layers close to the soil if the soil variables are part of the control vector (Figure $7.15 \mathrm{~b}$, third and fourth panel).

\subsubsection{Reduction of RMSE}

The benefit of the LST assimilation relative to the Control is estimated by the relative change of RMSE (rRMSE)

$$
r R M S E=\frac{R M S E-R M S E_{\mathrm{Control}}}{R M S E_{\mathrm{Control}}} \cdot 100 \%,
$$




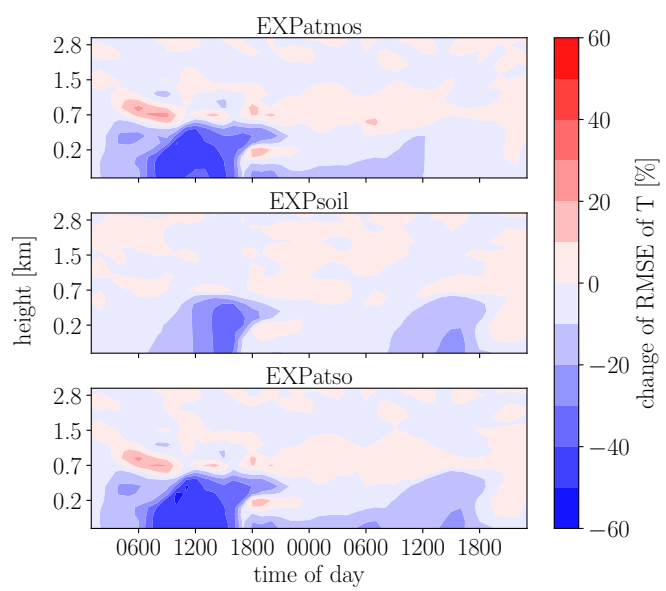

(a)

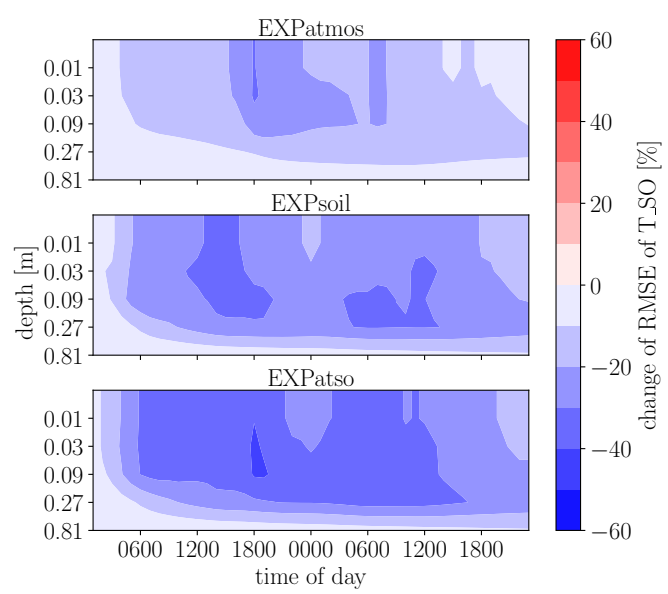

(b)

Figure 7.16: Evolution of relative change of RMSE of temperature of (a) the atmospheric boundary layer $(T)$ and of (b) the soil $\left(T_{\mathrm{so}}\right)$ from March 27 to March 29, 2017. First guess evaluated against truth averaged over the whole experiment domain. Shown are the rRMSE of the three experiments against Control. rRMSE of EXPatmos (top), rRMSE of EXPsoil (middle), rRMSE of EXPatso (bottom). Blue shaded areas indicate an improved RMSE of the experiment, red shaded areas indicate a deterioration. Figure taken from Sgoff et al. (2020).

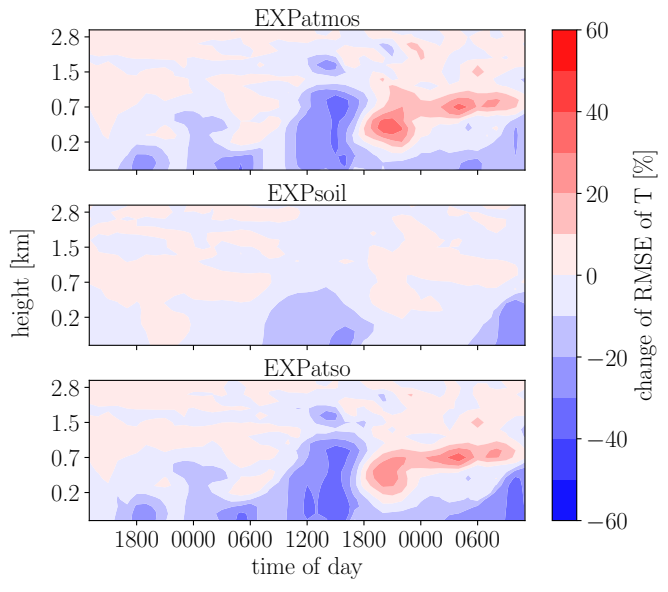

(a)

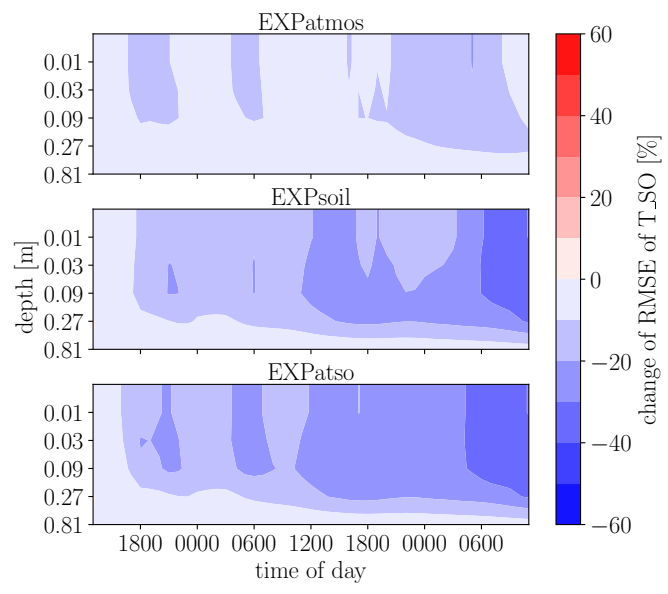

(b)

Figure 7.17: Like Figure 7.16 but from August 28 to August 30, 2017. Figure taken from Sgoff et al. (2020).

where $R M S E$ is the RMSE of the respective experiment and $R M S E_{\text {Control }}$ is the RMSE of the open loop (Control).

During the two-day early spring period, the RMSE of boundary layer temperature is reduced in all three experiments (Figure 7.16a), during daytime up to $60 \%$ near the ground. The greatest improvement of RMSE of EXPatmos is between 0600 UTC and 1200 UTC on March 27 (Figure 7.16a, top). The 
reduction of RMSE of EXPsoil is shifted compared to EXPatmos by approximately six hours through the indirect effect of the improved soil state and has its maximum during the afternoon (Figure 7.16a, middle). EXPatso combines successfully both improvements but also includes the increased RMSE of $E X$ Patmos at the top of the ABL (Figure 7.16a, bottom). The RMSE of the soil temperature is also reduced by the assimilation of LST, partly up to $50 \%$ (Figure 7.16b). The effect is best, if the coupled assimilation setting is performed (EXPatso). The deeper soil layers keep the positive effect over a longer period than the upper soil levels.

All in all, the effect of LST assimilation on near surface temperature during the two days of March is stronger during the first day because more LST observations were available and could be assimilated. On the second day, a cloud pattern over north-eastern Germany reduced the number of observations, and, consequently, the available LST information within the assimilation cycle (Figure 7.6a).

During the two-day summer period, the RMSE of boundary layer temperature is reduced in all three experiments as well. As in the two-day case in March the main reduction is during daytime, but with its maximum up to $40 \%$ in the afternoon (Figure7.17a). In the afternoon on August 29, the positive effect of LST assimilation on the atmospheric temperature reaches up to a height of $1.5 \mathrm{~km}$ within the experiments including the atmosphere in their control vector EXPatmos and EXPatso (Figure 7.17a, top and bottom). During the night from August 29 to August 30, EXPatmos and EXPatso show an increase of the RMSE of the atmospheric temperature located above the ABL. A different vertical localization during night and day could be an option to avoid this effect. The impact of LST assimilation on the RMSE of atmospheric temperature of EXPsoil is seen during daytime up to a height of $0.7 \mathrm{~km}$ and does not lead to an increase of RMSE (Figure 7.17a, middle).

The RMSE of the soil temperature is consistently reduced in each summer experiment. The RMSE decreases as the number of assimilated synthetic LST observations increases (Figure 7.6b and 7.17b). As in the early spring period the deeper soil layers keep the positive effect longer than the upper soil layers.

Besides temperature, moisture variables in the ABL and the soil are also influenced by the LST assimilation through cross-correlations. The direct effect of LST assimilation in EXPatmos and EXPatso during the March period reduces the atmospheric specific humidity clearly too much (Figure 7.18a, top and bottom). The RMSE of specific humidity within this two experiments reaches up to $70 \%$. This remarkable increase takes mainly place in the morning transition. The negative influence of LST assimilation can be due to inappropriate covariances. In contrast, the specific humidity of EXPsoil benefits from the assimilation of LST. On the afternoon of the second day of the experimental period, the RMSE of relative humidity is reduced by more than $10 \%$ (Figure 7.18a, middle). Because fewer LST observations are assimilated during this period an improvement due to the accumulated effects of an improved state of 


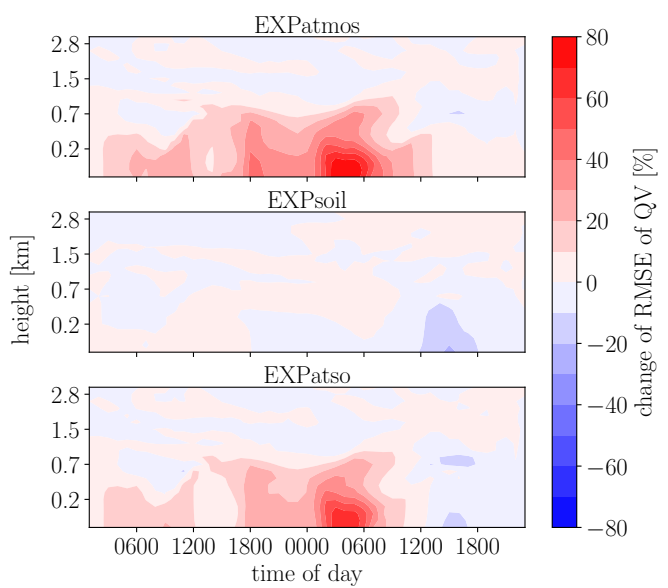

(a)

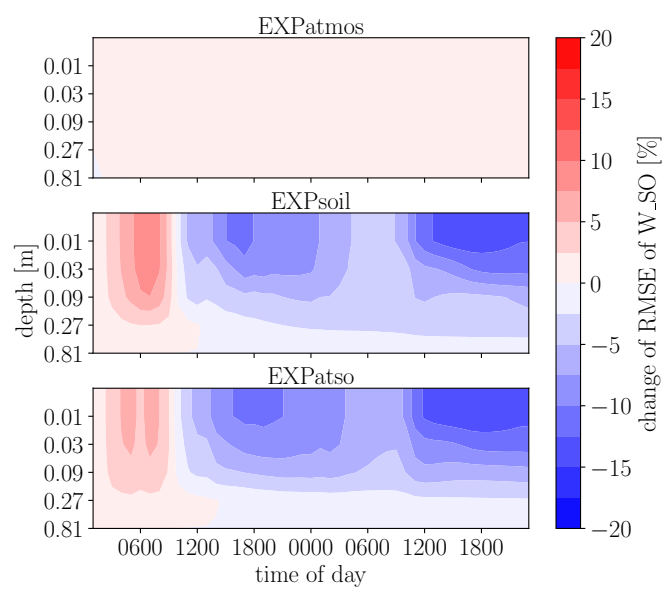

(b)

Figure 7.18: Like Figure 7.16 but the evolution of relative change of RMSE of (a) atmospheric specific humidity $(Q V)$ and of (b) soil moisture $\left(W_{\text {so }}\right)$ is shown. Figure taken from Sgoff et al. (2020).

the soil is assumed. This positive effect can also been seen in EXPatso in an attenuated form.

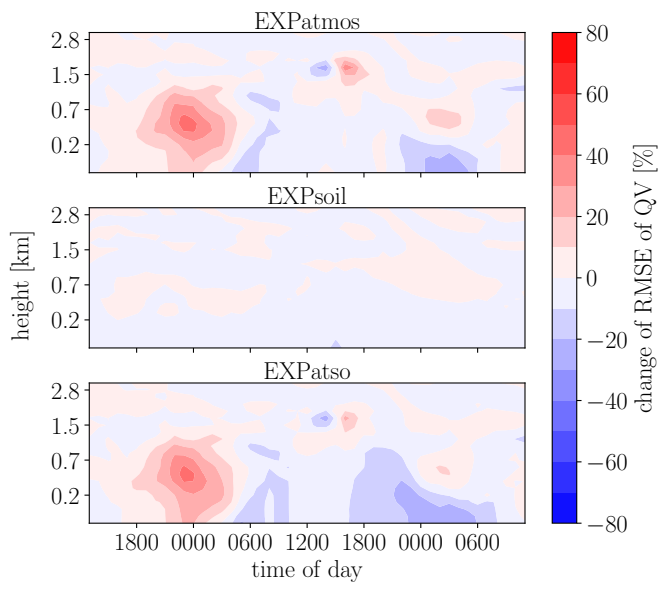

(a)

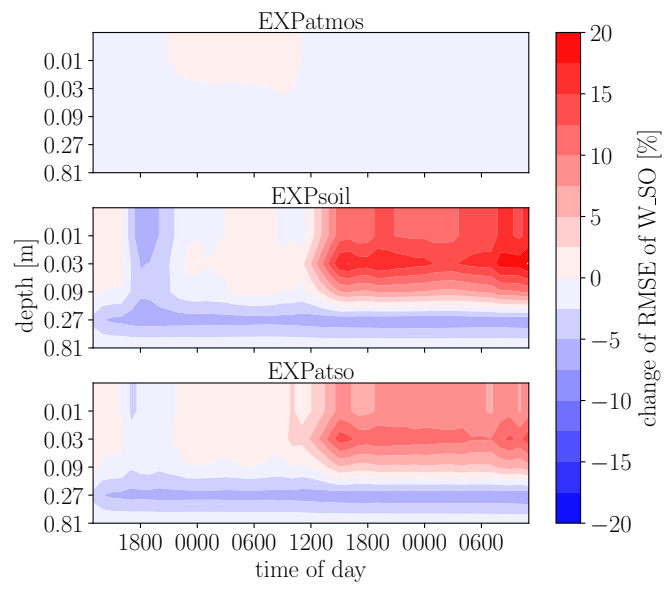

(b)

Figure 7.19: Like Figure 7.18 but from August 28 to August 30, 2017. Figure taken from Sgoff et al. (2020).

In contrast to the atmospheric humidity, the soil moisture can benefit from the direct effect of LST assimilation within the soil in March by introducing moisture (Figure 7.18b). Within the first six hours the assimilation of LST dries the soil, but after twelve hours of LST assimilation moisture is introduced into the soil by LST assimilation, which reduces the RMSE mainly during daytime by more than $12 \%$. This positive impact only occurs if the soil temperature and moisture are part of the control vector (Figure 7.18b, middle and bottom). In the case, where only the atmospheric variables are part of the control vector, the assimilation of LST has hardly an impact on the soil moisture. The RMSE of EXPatmos is slightly increased (less than $2.5 \%$ ), but mostly it is close to the 
Control (Figure 7.18b, top).

As in the early spring case, the RMSE of specific humidity of EXPatmos and EXPatso in the summer case increases during the morning transition, but only on the first day (Figure 7.19a, top and bottom). On the second day an the RMSE of specific humidity increases during the night and morning transition (Figure 7.19a, middle and bottom).

The difference between the soil moisture down to $0.5 \mathrm{~m}$ in the nature run and the assimilation experiments is smaller for the summer cases. The soil of the assimilation experiments is only about $5 \%$ drier than the nature run (not shown). The deeper layers of the assimilation experiments are more moist than the nature run, they were however unaffected by the LST assimilation. As in the spring case, the assimilation of LST introduces moisture into the upper soil levels after twelve hours of assimilation (Figure 7.19b, middle and bottom). The upper soil levels become too moist and only the deeper layers benefit from the additional moisture. As in early spring the RMSE of EXPatmos is nearly unaffected (Figure $7.19 \mathrm{~b}$, top).

\subsubsection{Impact on Surface and Near Surface Variables}

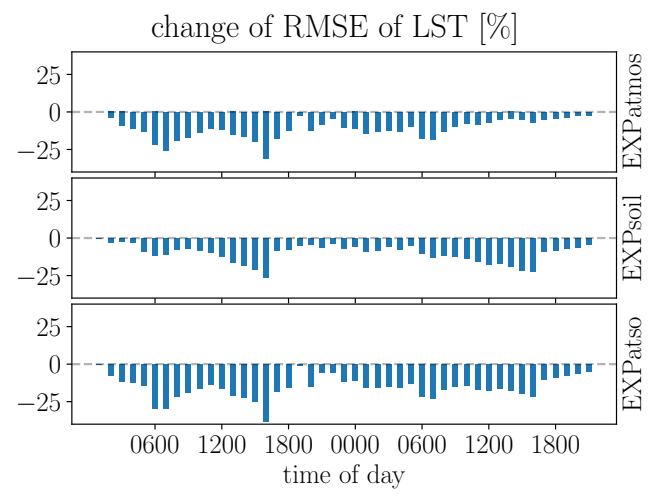

(a)

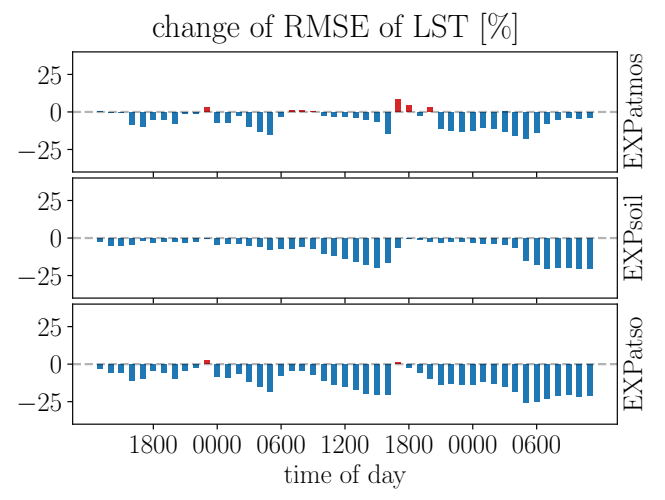

(b)

Figure 7.20: (a) Evolution of relative change of RMSE of LST ( $\left.T_{\mathrm{g}}\right)$ during the March and (b) during the August, 2017 experimental period. First guess evaluated against nature run averaged over the whole experiment domain. Shown are the rRMSE of the three control vector experiments (Table 7.1) against Control. (top) rRMSE of EXPatmos, (middle) rRMSE of EXPsoil, (bottom) rRMSE of EXPatso. Blue bars indicate a reduced RMSE of the experiment and red bars an increased RMSE.

In comparison with the Control the assimilation of LST has a positive impact on the first guess of LST of all three control vector experiments during the two-day case studies in early spring and summer (Figure 7.20). The reduction of RMSE of LST depends on daytime and on observation ability. During night the RMSE reduction is with around 5\% to $10 \%$ smaller than the RMSE reduction during day with more than $10 \%$. During the early spring period 


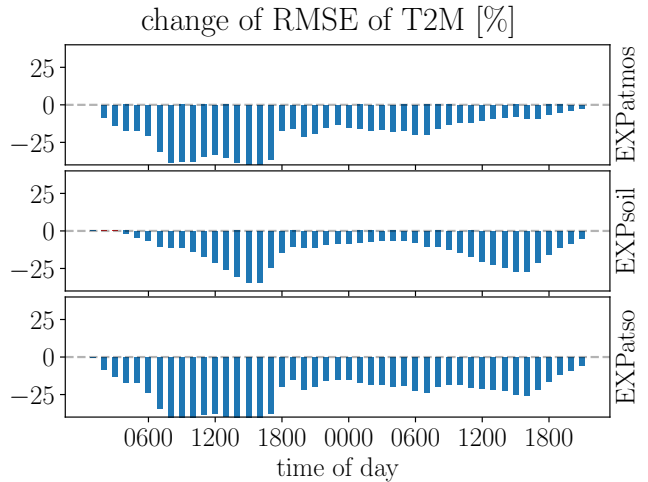

(a)

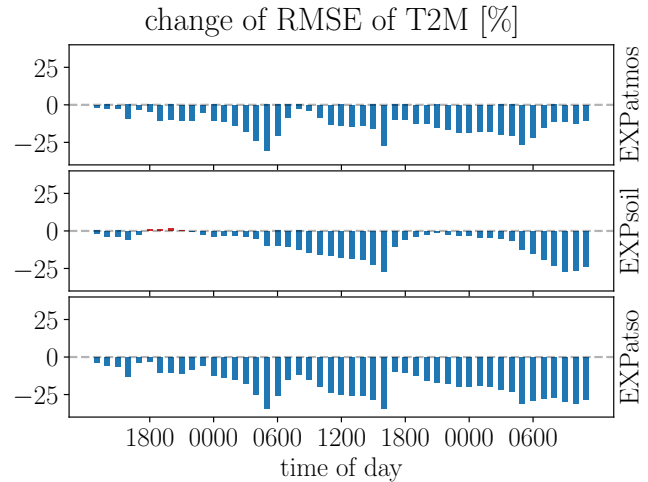

(b)

Figure 7.21: As Figure 7.20 but for $2 \mathrm{~m}$-temperature (T2M).

peaks of RMSE reduction of LST up to more than $25 \%$ are reached. Around 1800 UTC the RMSE reduction in the two-day case studies is lowest. The reduced availability of LST observations on the second day of the March case study (Figure 7.6a) leads to a lower RMSE reduction of EXPatmos. In the other March experiments, EXPsoil and EXPatso, the lower number of observations is less noticeable because the soil still contains information derived from former LST assimilation (Figure 7.20a, 7.16b and 7.18b). During the August period more LST observations are available on the second day (Figure 7.6b) and the RMSE reduction of LST is as well stronger on the second day.

The impact on the temperature at 2 metre height by LST assimilation is positive as well (Figure 7.21). The RMSE of the experiments is reduced over the whole experiment periods of March and August partly by up to $40 \%$. In EXPatmos of early spring and summer the reduction of RMSE depends mainly on the availability of LST observations. The more LST observations there are, the more the RMSE of $2 \mathrm{~m}$-temperature is reduced (Figure 7.6 and 7.21, top panels). In EXPsoil the reduction of 2 m-temperature RMSE depends on the daytime as well. A greater improvement in the $2 \mathrm{~m}$-temperature can be observed in March and August, 2017 during the day (Figure7.21, middle panels). Both positive effects of EXPatmos and EXPsoil are combined in EXPatso (Figure 7.21, bottom panels).

The sensible heat flux depends mainly on the temperatures near and at the land surface (Equation 5.4). These temperatures were improved by the assimilation of LST, especially during daytime (Figure 7.16a, 7.16b, 7.17a and $7.17 \mathrm{~b}$. This improvement during the day is passed on to the sensible heat flux in EXPsoil and EXPatso of both case studies. The RMSE of sensible heat flux of both experiments during the March period is reduced by up to $25 \%$ (Figure 7.22a, middle and bottom). During August the effect is smaller and the reduction of RMSE in EXPsoil and EXPatso is up to $10 \%$ (Figure $7.22 \mathrm{~b}$, middle and bottom). In contrast, the RMSE of the sensible heat flux in EXPatmos improves and deteriorates between $0 \%$ to $10 \%$ (Figure 7.22 , top panel). This shows that the lack of direct information on the soil is clearly reflected in the surface energy budget. 


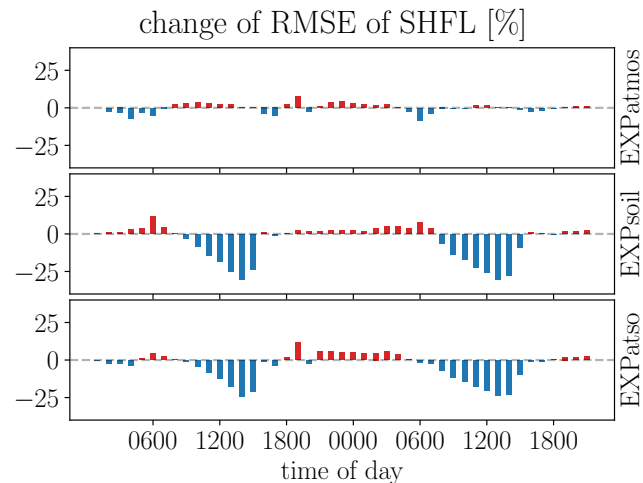

(a)

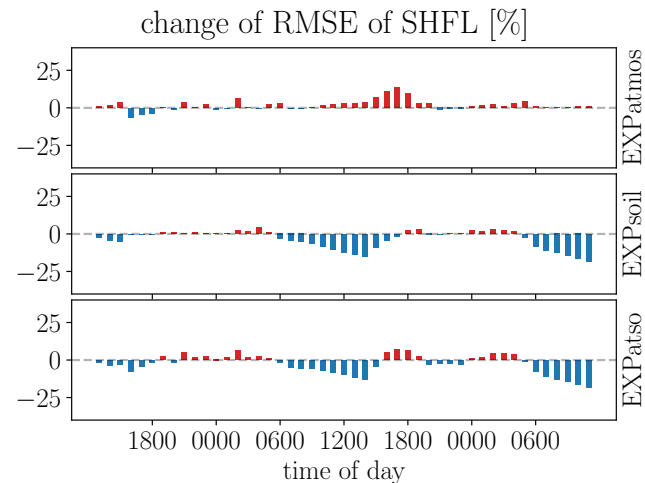

(b)

Figure 7.22: As Figure 7.20 but for sensible heat flux (SHFL).

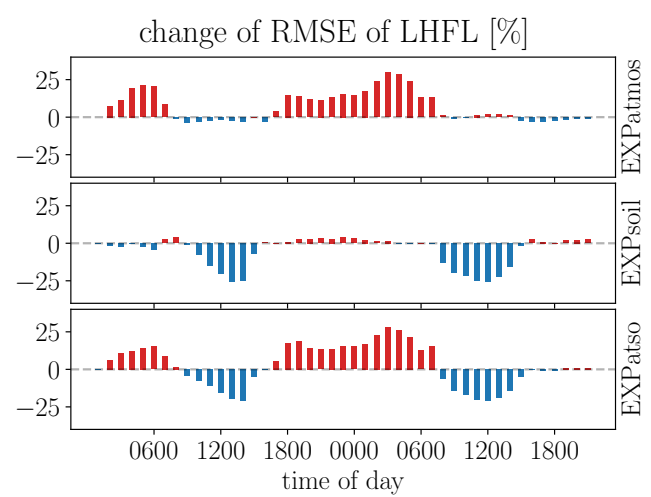

(a)

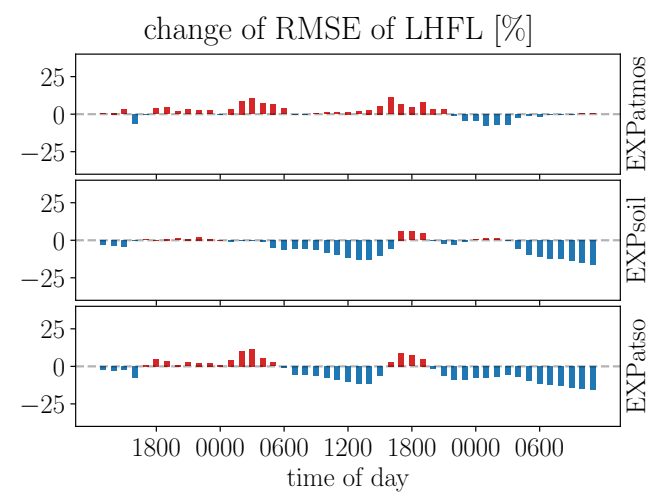

(b)

Figure 7.23: Like Figure 7.20 but for latent heat flux (LHFL).

The influence of assimilation of LST on the latent heat flux depends as well on the control vector setup. In EXPatmos of both case studies the impact of LST assimilation is more pronounced at night and worsens the RMSE of the latent heat flux by up to $25 \%$ in March and up to $10 \%$ in August (Figure 7.23. top panels). In EXPsoil, however, the influence on the latent heat flux is more pronounced during the day and reduces its RMSE by up to $25 \%$ in March and up to $10 \%$ in August (Figure 7.23, middle panels). EXPatso, which contains soil and atmospheric variables in the control vector, combines both, the positive and the negative effects. During the day the RMSE is reduced by the assimilation of LST and at night the RMSE grows through the assimilation of LST (Figure 7.23 , bottom panels). The latent heat flux at the surface depends mainly on the atmospheric humidity and the soil surface moisture (Equation 5.5). During March the atmospheric humidity mainly suffers from the assimilation of LST (Figure 7.18a). At the same time the soil moisture was improved by the LST assimilation (Figure $7.18 \mathrm{~b}$ ). This negative and positive influence was passed on to the latent heat flux (Figure 7.23a). During daytime the positive influence predominates and at night the negative influence predominates. During the two-day August period the impact is smaller than during March (Figure 7.23a). Including the soil into the control vector improves the first guess of latent heat 
flux during day as well, although the RMSE of soil moisture increases due to the assimilation of LST (Figure 7.19b).

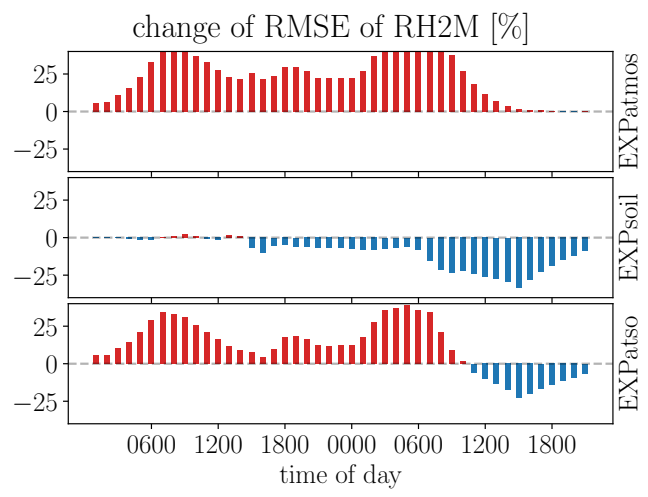

(a)

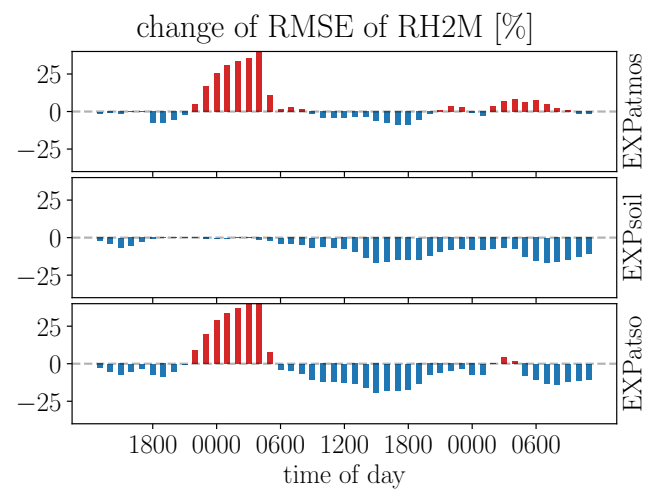

(b)

Figure 7.24: As Figure 7.20 but for 2 m-relative humidity (RH2M).

The change of RMSE of relative humidity in $2 \mathrm{~m}$ height coincides with the change of RMSE of specific humidity of the lower atmospheric model levels (Figure 7.18a, 7.19a and 7.24). The deterioration in March and August comes mainly from the direct influence of LST assimilation on the atmosphere ( $E X$ Patmos and EXPatso). While the main improvement in both case studies comes mainly from the direct influence of LST assimilation on the soil (EXPsoil and EXPatso) although the soil moisture in August has an increased RMSE.

To summarize, the impact of LST assimilation on the variables of the near surface and the energy budget at the surface was stronger if more LST observations were available and could be assimilated. Especially during daytime, the impact of assimilation of LST improved the first guess, but during nighttime the positive impact is smaller or with regard to atmospheric humidity and corresponding surface fluxes even negative (Figure 7.18a and 7.23).

\subsubsection{Evaluation of 24-hour Forecasts}

All in all, the assimilation of LST led to improved initial conditions for the ABL and the upper soil of the forecast model. To investigate the effect of LST assimilation on the COSMO-model forecast, a 24-hour forecast was simulated every six hours. The assimilation free forecasts were initialised from Control and EXPatso of the two-day experiments in March and August, 2017.

As illustrated in Figure 7.25a, the LST assimilation during the two-day study in March 2017 based on EXPatso has a positive impact on the temperature of the ABL. In the EXPatso forecast started at 0600 UTC the RMSE is reduced by over $20 \%$ in the first ten forecast hours within the lower levels of the atmosphere up to $0.7 \mathrm{~km}$ (Figure 7.25a top). In contrast, at the top of the ABL, the first three forecast hours have an increased RMSE about 10\% to 20\%. After 12 forecast hours the reduction of RMSE is negligible. 


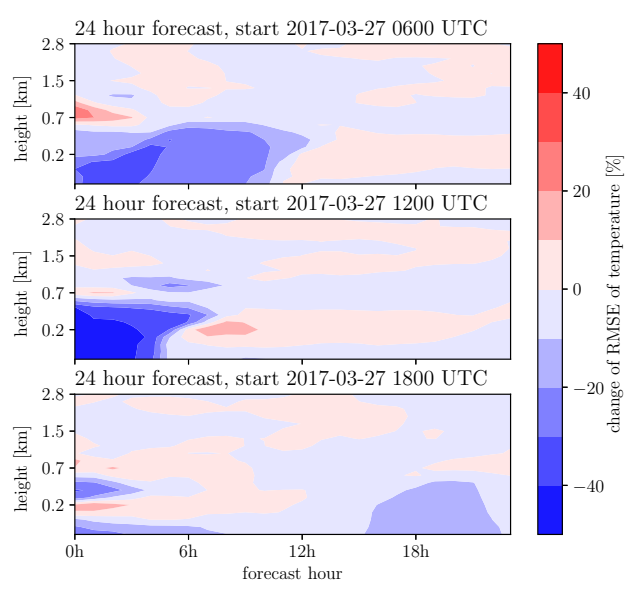

(a)

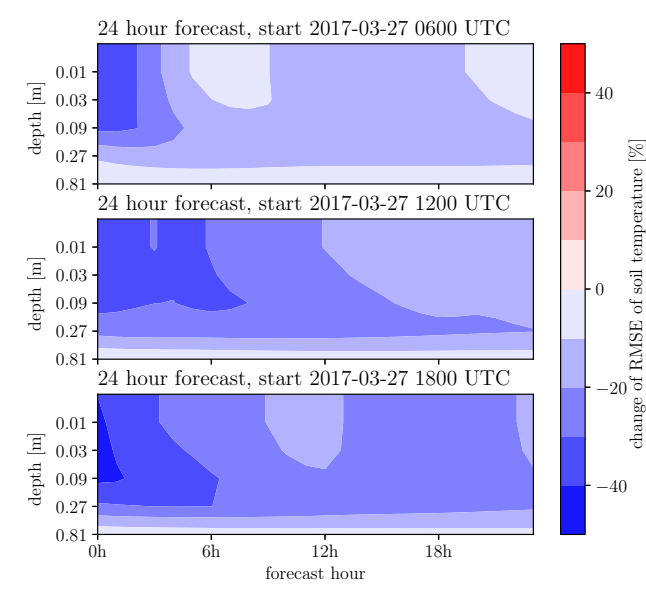

(b)

Figure 7.25: (a) Change of rRMSE of the early spring case for three free 24-hour forecasts of temperature of atmospheric boundary layer (b) and of soil levels. (Top) One started after six hours of LST assimilation, (middle) one after 12 hours of LST assimilation and (bottom) one after 18 hours of LST assimilation (lower panels). The forecast started from EXPatso and the Control are evaluated against the truth averaged over the whole experiment domain. Shown is the rRMSE of EXPatso against Control. Blue shaded areas indicate an improved RMSE of EXPatso and red shaded areas indicate a deterioration. Figure taken from Sgoff et al. (2020).

The forecast started on March 27 at 1200 UTC has also an improved ABL temperature because of LST assimilation (Figure 7.25a, middle). The positive effect on ABL temperature lasts for four hours near to the surface and for six hours between 0.2 to $0.7 \mathrm{~km}$. After six hours of forecast to nine hours of forecast, around the evening transition, the ABL temperature RMSE is $10 \%$ larger than of Control. This negative effect is reduced to around $3 \%$ after nine hours of forecast. The forecast started on March 27 at 1800 UTC shows only short-lasting improvements of LST assimilation (Figure 7.25a, bottom). Because of the growing cloud cover less LST observations were available that results in a smaller impact of LST assimilation. Around 18 hours of forecast a RMSE reduction of $15 \%$ occurs, possibly due to the indirect effect of improved soil variables.

In general, the soil temperature forecast is improved by the LST assimilation over each soil level down to $1 \mathrm{~m}$ (Figure 7.25b). The soil temperature RMSE of the forecast started at $0600 \mathrm{~h}$ is mainly reduced during the first three forecast hours within the upper $0.1 \mathrm{~m}$ of the soil. The forecast started at $1200 \mathrm{~h}$ includes an even bigger reduction of soil temperature RMSE up to six hours down to $0.5 \mathrm{~m}$. A reduction of soil temperature RMSE around $20 \%$ lasts for the whole 24-hour forecast, i.e. the soil temperature keeps the effect of LST assimilation longer than the ABL temperature. The reduction of soil temperature RMSE over $20 \%$ remains over the whole 24 hours of the forecast started at $1800 \mathrm{~h}$. 


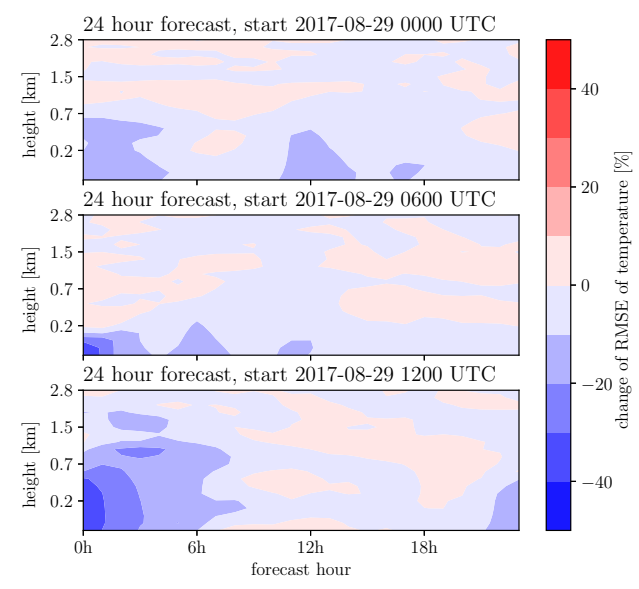

(a)

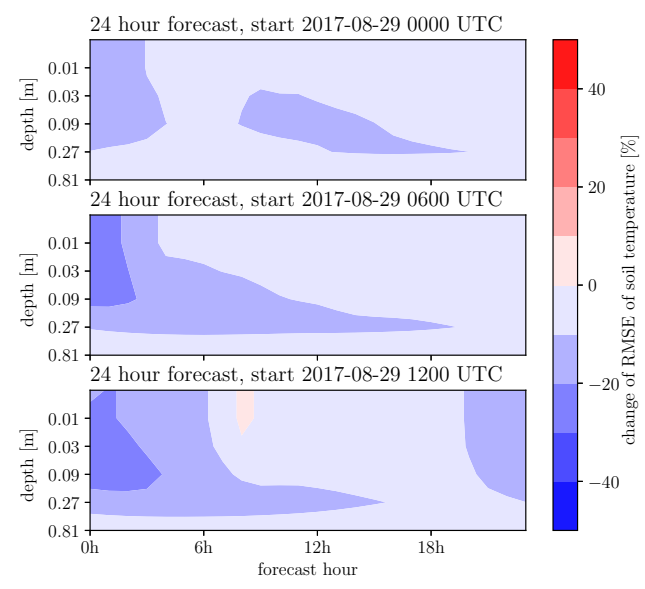

(b)

Figure 7.26: As Figure 7.25 but forecasts of the August case. Figure taken from Sgoff et al. (2020).

The 24-hour forecasts of atmospheric temperature and soil temperature based on EXPatso of the two-day study in August 2017 also benefit from the LST assimilation (Figure 7.26a), but the benefit is less pronounced than during the two-day study in March 2017. The first four forecast hours of the temperature of the ABL started at 0000 UTC have a $10 \%$ reduced RMSE and a further reduction of more than $10 \%$ around 12 hours of forecast (Figure $7.26 \mathrm{a}$, top).

In the EXPatso forecast started 0600 UTC, the RMSE of the atmospheric temperature is reduced over the first 12 hours (Figure 7.26a, middle). The reduction of the RMSE up to the height of $0.2 \mathrm{~km}$ ranges between $10 \%$ and $30 \%$ (only first forecast hour). The forecast started on August 29 at 1200 UTC profits from the LST assimilation during the first six forecast hours. The forecast of the atmospheric temperature is improved up to a height of $1.5 \mathrm{~km}$ (Figure $7.26 \mathrm{a}$, bottom). After 22 hours a RMSE reduction of $15 \%$ arises. This reduction coincides with an improvement of soil temperature of the same forecast which indicates that the soil may have a positive effect on the atmosphere over the whole 24-hour forecast.

As in March, the soil temperature forecast is also improved in August. The assimilation of LST reduces the RMSE of the forecast of the soil temperature down to $1 \mathrm{~m}$ with the main reduction occurring in the first three forecast hours (Figure 7.26b). The forecasts started at 0600 UTC and 1200 UTC keep the reduction of the RMSE of soil temperature longer than the forecast started 0000 UTC, especially in the deeper layers between $0.1 \mathrm{~m}$ and $0.3 \mathrm{~m}$ (Figure $7.26 \mathrm{~b}$, middle and bottom). After 20 forecast hours from forecast started at 1200 UTC the reduction of RMSE of soil temperature increases again (Figure 7.26b, bottom).

To summarize, the soil temperature, especially the levels between 0.1 to $0.3 \mathrm{~m}$, keep the positive impact of LST assimilation longer in the forecast than the ABL temperature. The soil moisture also keeps the positive or negative 
impact longer than the specific humidity of the ABL (not shown).

\subsubsection{Summary}

The experiments show that the assimilation of LST reduces the RMSE of atmospheric and soil temperature. During daytime this positive impact is even stronger than during nighttime. In the experiments with the weakly coupled data assimilation system the positive impact is mainly restricted on the part of the model, atmosphere or soil, whose prognostic variables are part of the control vector. The fully coupled COSMO-KENDA-system combines this impacts and thus outperforms the weakly coupled assimilation systems restricted to soil or atmosphere only. The effect of LST assimilation on the atmospheric humidity and the soil moisture is both, positive and negative. This indicates that the background cross-correlations of the LETKF between the synthetic LST observations and the moisture variables are not appropriately represented. 


\section{Chapter 8}

\section{Ensemble Consistency Score}

The assimilation of LST proves to be differently successful (Chapter 7). The impact of LST assimilation depends on time of day and whether the temperature or humidity variables are evaluated. A possible reason for these differences could be spurious correlations between the synthetic LST observation and the related temperature and moisture variables. Therefore, it is of high interest to determine if the ensemble covariances are able to represent the model correlations of the nature run. The evaluation of background error covariances is a separate area of research and a detailed review was presented by Bannister (2008a b) . The aim of this chapter is to introduce a numerical indicator, which provides information about the quality of the ensemble covariances between two variables in a simple way. Thus, a dimensionless value $q$ - from now on named ensemble consistency score - is derived in Section 8.1. Its suitability to indicate whether the covariances of the ensemble at a certain grid point match the true covariances of the nature run at the same point or not is evaluated with a Lorenz 1963 system (Section 8.2.2) and with the known truth of the OSSEs of this thesis (Section 8.3.2).

\subsection{Derivation}

The analysis step of the assimilation cycle depends on appropriate observation and background error covariances. This thesis is based on an OSSE setup, therefore the observation errors are known by definition. Consequently, the focus is now on the background error covariances $\mathbf{P}^{\mathrm{b}}$. The aim is to introduce an ensemble consistency score $q$, which indicates accurate background error covariances within the fully coupled land-atmosphere assimilation system if $q$ is positive and incorrect background error covariances if $q$ is negative. The most popular study about the diagnosis of data assimilation error statistics is by Desroziers et al. (2005). Within their study they defined consistency diagnostics of errors in observational space based on the information of innovations in the observational space. Their consistency diagnostic on background errors is based on the condition that if the background error covariances $\mathbf{P}^{\mathrm{b}}$ and observation error covariances $\mathbf{R}$ in observational space correspond to the true error covariances then

$$
E\left[H\left(\mathbf{x}^{\mathrm{a}}\right)-H\left(\mathbf{x}^{\mathrm{b}}\right)\left(\mathbf{y}-H \mathbf{x}^{\mathrm{b}}\right)^{T}\right]=\mathbf{H P}^{\mathrm{b}} \mathbf{H}^{T} .
$$


Here, $E$ equals a statistical expectation operator, $H$ is the nonlinear observation operator and $\mathbf{H}$ the observation operator matrix derived from the linearized $H$. The background state is given by the vector $\mathrm{x}^{\mathrm{b}}$, the analysed state by $\mathrm{x}^{\mathrm{a}}$ and the observations by the vector $\mathbf{y}$.

Within this thesis, the consistency check of background error covariances is based on the information of the true state itself, because the truth provided by the OSSE can be used to verify the background error covariances of the model ensemble. Therefore, the score $q$, gives insight in the quality of the model ensemble and further can give information about the expected analysis: if the background error covariances are consistent with the nature run the impact on the analysis is positive and vice versa. In the following, the analysis step is considered as correct if the assimilation leads to an analysis ensemble mean $\bar{x}^{\mathrm{a}}$, which is closer to the truth $x^{\text {true }}$ than the mean of the background ensemble $\bar{x}^{\text {b }}$ (Figure 8.1).

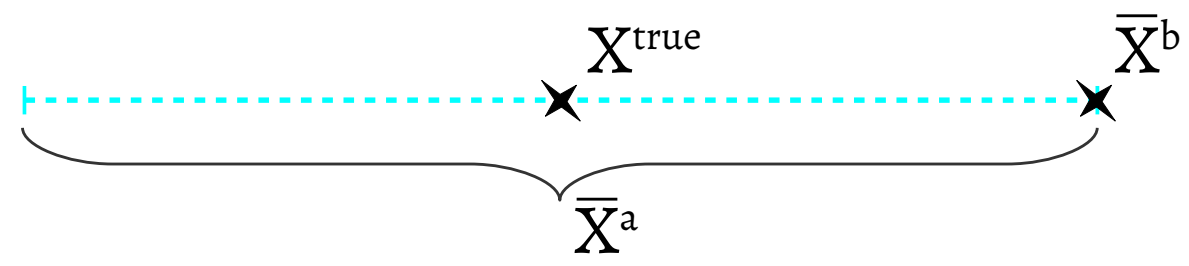

Figure 8.1: If the mean of the analysis ensemble $\bar{x}^{\mathrm{a}}$ is within the blue area, then Equation 8.2 is fulfilled and the analysis step at the certain grid point is successful. $x^{\text {true }}$ is the true value and $\bar{x}^{\mathrm{b}}$ the mean of the background ensemble.

This condition can be checked at each grid point for each variable $i$ of the control vector

$$
\left|\bar{x}_{i}^{\mathrm{a}}-x_{i}^{\mathrm{true}}\right|<\left|\bar{x}_{i}^{\mathrm{b}}-x_{i}^{\mathrm{true}}\right|,
$$

where $\bar{x}_{i}^{\mathrm{a}}$ is the ensemble mean of the analysis of the variable, $\bar{x}_{i}^{\mathrm{b}}$ is the ensemble mean of the background of the variable and $x_{i}^{\text {true }}$ is the true value of the variable. As in the work of Desroziers et al. (2005), statistical linear estimation theory is used as the initial equation to derive the score $q$. The analysis $\overline{\mathbf{x}}^{\mathrm{a}}$ is given by

$$
\overline{\mathbf{x}}^{\mathrm{a}}=\overline{\mathbf{x}}^{\mathrm{b}}+\mathbf{P}^{\mathrm{b}} \mathbf{H}^{T}\left(\mathbf{R}+\mathbf{H P}^{\mathrm{b}} \mathbf{H}^{T}\right)^{-1}\left(\mathbf{y}-\mathbf{H} \overline{\mathbf{x}}^{\mathrm{b}}\right),
$$

where $\mathbf{P}^{\mathrm{b}}$ equals the background error covariance matrix, $\mathbf{y}$ is the observation, $\mathbf{H}$ equals the linearisation of the observation operator, and $\mathbf{R}$ equals the observation error covariance matrix. Background error covariances between different variables can be correlated. This effect can be seen by focusing on the covariances between one observed variable $(i=1)$ and any model variable that relates to the observed variable $(i=2)$, hence the observation operator $\mathbf{H}$ is simplified as $H_{0}=(1,0)$. The background error covariance matrix $\mathbf{P}^{\mathrm{b}}$ is a 2x2 matrix derived from the background ensemble and $\mathbf{R}$ is the observation error variance and thus assumed as a constant value $R_{0}$. Including these 
simplifications, Equation 8.3 reads as

$$
\left(\begin{array}{l}
\bar{x}_{1}^{\mathrm{a}} \\
\bar{x}_{2}^{\mathrm{a}}
\end{array}\right)=\left(\begin{array}{l}
\bar{x}_{1}^{\mathrm{b}} \\
\bar{x}_{2}^{\mathrm{b}}
\end{array}\right)+\left(\begin{array}{ll}
b_{11} & b_{21} \\
b_{21} & b_{22}
\end{array}\right)\left(\begin{array}{l}
1 \\
0
\end{array}\right)\left[R_{0}+(1,0)\left(\begin{array}{ll}
b_{11} & b_{21} \\
b_{21} & b_{22}
\end{array}\right)\left(\begin{array}{l}
1 \\
0
\end{array}\right)\right]^{-1}\left[y-(1,0)\left(\begin{array}{l}
\bar{x}_{1}^{\mathrm{b}} \\
\bar{x}_{2}^{\mathrm{b}}
\end{array}\right)\right],
$$

which can be simplified to

$$
\left(\begin{array}{l}
\bar{x}_{1}^{\mathrm{a}} \\
\bar{x}_{2}^{\mathrm{a}}
\end{array}\right)=\left(\begin{array}{l}
\bar{x}_{1}^{\mathrm{b}} \\
\bar{x}_{2}^{\mathrm{b}}
\end{array}\right)+\left(\begin{array}{l}
b_{11} \\
b_{21}
\end{array}\right)\left(R_{0}+b_{11}\right)^{-1}\left(y-\bar{x}_{1}^{\mathrm{b}}\right) .
$$

This equation can be divided into its independent components. As a reminder, $i=1$ indicates the observation or alternatively the appropriate model equivalent of the observed value and $i=2$ indicates the related model variable.

$$
\begin{aligned}
& \bar{x}_{1}^{\mathrm{a}}=\bar{x}_{1}^{\mathrm{b}}+\frac{b_{11}}{R_{0}+b_{11}}\left(y-\bar{x}_{1}^{\mathrm{b}}\right), \\
& \bar{x}_{2}^{\mathrm{a}}=\bar{x}_{2}^{\mathrm{b}}+\frac{b_{21}}{R_{0}+b_{11}}\left(y-\bar{x}_{1}^{\mathrm{b}}\right) .
\end{aligned}
$$

The further focus is on Equation 8.6, because it includes the ensemble covariances between the observation and the corresponding model value. For example, $\bar{x}_{1}^{\mathrm{b}}$ is the ensemble mean of the simulated LST, $y$ is the synthetic LST observation and $\bar{x}_{2}^{\mathrm{b}}$ is the ensemble mean of the specific humidity of the lowest atmospheric model level. The difference between the analysis $\bar{x}_{2}^{\mathrm{a}}$ and the background $\bar{x}_{2}^{\mathrm{b}}$ in model space can be written as:

$$
\bar{x}_{2}^{\mathrm{a}}-\bar{x}_{2}^{\mathrm{b}}=\frac{b_{21}}{R_{0}+b_{11}}\left(y-\bar{x}_{1}^{\mathrm{b}}\right) .
$$

Bannister (2008a) derived this equation in a more general form

$$
x_{l}^{\mathrm{a}}-x_{l}^{\mathrm{b}}=b_{l k} \frac{y-x_{k}^{\mathrm{b}}}{b_{k k}+R_{0}}
$$

Bannister, 2008a, Equation 12) to describe how the $\mathbf{P}^{\mathrm{b}}$ of the observed component ( $k$-th component) and the $l$-th component of the control vector affects the analysis. Equation 8.7 and 8.8 show that although only one observation $y$ of the model state is included, the state of the component is also changed. Hence, the observation information is distributed in the model space via the background error covariance matrix $\mathbf{P}^{\mathrm{b}}$. Accordingly, a mismatching $\mathbf{P}^{\mathrm{b}}$ can cause spurious correlations between the variables and consequently these errors are passed on to the not observed components. In contrast, a consistent $\mathbf{P}^{\mathrm{b}}$ improves the analysis. To examine the consistency of $\mathbf{P}^{\mathrm{b}}$ within this thesis the nature run is used.

The product between the difference of the analysis and the background mean $\left(\bar{x}_{2}^{\mathrm{a}}-\bar{x}_{2}^{\mathrm{b}}\right)$ and the difference between the true state and the background mean $\left(x_{2}^{\text {true }}-\bar{x}_{2}^{\mathrm{b}}\right)$ is

$$
\left(x_{2}^{\text {true }}-\bar{x}_{2}^{\mathrm{b}}\right)\left(\bar{x}_{2}^{\mathrm{a}}-\bar{x}_{2}^{\mathrm{b}}\right)=\left(x_{2}^{\mathrm{true}}-\bar{x}_{2}^{\mathrm{b}}\right) \frac{b_{21}}{R_{0}+b_{11}}\left(y-\bar{x}_{1}^{\mathrm{b}}\right) .
$$


If the model state is pulled by the analysis step into the direction of the true state, the left hand side of Equation 8.9 is positive, i.e. the right hand side of Equation 8.9 necessarily is positive and guarantees a successful analysis step:

$$
\left(x_{2}^{\text {true }}-\bar{x}_{2}^{\mathrm{b}}\right) \frac{b_{21}}{R_{0}+b_{11}}\left(y-\bar{x}_{1}^{\mathrm{b}}\right)>0 .
$$

The background error covariances are defined by

$$
b_{21}=\frac{1}{L-1} \sum_{l=1}^{L}\left(x_{1}^{1}-\bar{x}_{1}^{\mathrm{b}}\right)\left(x_{2}^{\mathrm{l}}-\bar{x}_{2}^{\mathrm{b}}\right)
$$

and

$$
b_{11}=\frac{1}{L-1} \sum_{l=1}^{L}\left(x_{1}^{1}-\bar{x}_{1}^{\mathrm{b}}\right)^{2} .
$$

The model ensemble consists of $L$ ensemble members, here $L=40$ was chosen to remain consistent with the OSSE. Based on Equation 8.10 the first version of the score $q$ can be deduced:

$$
q_{1}=\frac{\frac{1}{L-1} \sum_{l=1}^{L}\left(x_{2}^{\text {true }}-\bar{x}_{2}^{\mathrm{b}}\right)\left(x_{1}^{\mathrm{l}}-\bar{x}_{1}^{\mathrm{b}}\right)\left(x_{2}^{\mathrm{l}}-\bar{x}_{2}^{\mathrm{b}}\right)\left(y-\bar{x}_{1}^{\mathrm{b}}\right)}{R_{0}+\frac{1}{L-1} \sum_{l=1}^{L}\left(x_{1}^{\mathrm{l}}-\bar{x}_{1}^{\mathrm{b}}\right)^{2}} .
$$

If $q_{1}$ is positive the ensemble is moved by the analysis step in the correct direction, but $q_{1}$ also includes the possibility that the analysis step pushes the ensemble to far in the correct direction. Hence, this behaviour induces wrong ensemble covariances as well. To take care of this problem $q_{1}$ and thus the left hand side of Equation 8.9 as well is normalized:

$$
\frac{\bar{x}_{2}^{\mathrm{a}}-\bar{x}_{2}^{\mathrm{b}}}{x_{2}^{\text {true }}-\bar{x}_{2}^{\mathrm{b}}}=\frac{q_{1}}{\left(x_{2}^{\text {true }}-\bar{x}_{2}^{\mathrm{b}}\right)^{2}}=q_{\text {norm }} .
$$

Considering Equation 8.2 (illustrated by Figure 8.1 ) the difference between $\bar{x}_{2}^{\mathrm{a}}$ and $x_{2}^{\text {true }}$ must not be more than twice as large as the difference between $\bar{x}_{2}^{\mathrm{b}}$ and $x_{2}^{\text {true }}$ :

$$
0 \leq \frac{\bar{x}_{2}^{\mathrm{a}}-\bar{x}_{2}^{\mathrm{b}}}{x_{2}^{\text {true }}-\bar{x}_{2}^{\mathrm{b}}} \leq 2 .
$$

Only then the assimilation step is considered as successful at this point, i.e. a consistent covariance between the ensemble and the truth is represented by all values of $q_{\text {norm }}$ which fulfill $0 \leq q_{\text {norm }} \leq 2$. Using an adjusted parabola

$$
q=1-\left(q_{\text {norm }}-1\right)^{2}
$$

a positive $q$ represents exactly $0 \leq q_{\text {norm }} \leq 2$.

The ensemble consistency score $q$ can be seen as a score which forecasts if the assimilation step works well based on the knowledge gained from the ensemble covariances. If $q$ is positive the correlations are correctly represented and the assimilation of the observations improves the related model state. If $q$ is negative, the assimilation step leads to a deterioration of the related model state. The score $q$ does not indicate whether the variables are positively or negatively correlated. The score $q$ indicates if the correlation of the ensemble matches the truth. 


\subsection{Lorenz 1963 System Experiments}

\subsubsection{Experimental Setup}

To examine the ensemble consistency score $q$ derived in Section 8.1, test cases were conducted with a Lorenz 1963 system based on an OCTAVE code example of Nakamura and Potthast (2015) described in Appendix A. The nature runs of the test cases were generated by the three coupled ordinary differential equations of the Lorenz 1963 system equation. The state of the nature run is given by $\mathbf{x}=\left(x_{1}, x_{2}, x_{3}\right)^{T} \in \mathbb{R}^{3}$. Every nature run was started from $\mathbf{x}_{\text {init }}=(0,-10,21)$ and calculated $N_{\text {cycle }}$ time steps into the future. The nature run was assumed as the truth and each time step $t_{j}$ with $j=1, \ldots, N_{\text {cycle }}$ with time interval $d t=0.1$ the first component of the state was observed. For that purpose an observation $y$ was calculated by $y=H \mathbf{x}+\epsilon$ with the observation operator $H_{\mathrm{NR}}=H_{0}=(1,0,0)$ and a random noise perturbation $\epsilon$. Furthermore, at each time step $t_{j}$ the assimilation step was performed and the score $q$ was derived for the evaluation of the covariance between the observed state component $x_{1}=\mathbf{x}(1)$ and the second state component $x_{2}=\mathbf{x}(2)$ as $q_{2}$ and between $x_{1}=\mathbf{x}(1)$ and the second state component $x_{3}=\mathbf{x}(3)$ as $q_{3}$. The assimilation step was performed

Table 8.1: Setup of Lorenz 1963 experiments on the ensemble consistency score $q$. Dependencies of $H$ and $R$ are evaluated. The observation operator $H$ of the analysis step either corresponds to the observation operator $H_{0}$ from the calculation of $q$ or it deviates slightly from $H_{0}: H=(h, 0,0)$ with $h=\{0.80,0.81, \ldots, 1.19,1.20\}$. For the observation error $R$ also applies: either it corresponds to the observation error $R_{0}$ from the computation of $q$ or it differs slightly: $R \equiv r=\{0.01,0.02, \ldots, 0.29,0.30\}$.

\begin{tabular}{|c|c|c|c|c|}
\hline \multirow{2}{*}{ Exp } & \multicolumn{2}{|c|}{ Assimilation Cycle } & \multicolumn{2}{c|}{ Calculation of $q$} \\
\cline { 2 - 5 } & obs. operator $H$ & obs. error $R$ & obs. operator $H_{0}$ & obs. error $R_{0}$ \\
\hline \hline 1 & $H_{0}$ & $R_{0}$ & $H_{0}$ & $R_{0}$ \\
\hline 2 & $H=(h, 0,0)$ & $R_{0}$ & $H_{0}$ & $R_{0}$ \\
\hline 3 & $H_{0}$ & $R \equiv r$ & $H_{0}$ & $R_{0}$ \\
\hline
\end{tabular}

by an ensemble Kalman filter (see Section 2.1) with a 40-member ensemble. To reproduce the difference between the model and the truth, the Lorenz 1963 system used for the model forecast step of the data assimilation cycle differed from the Lorenz 1963 model of the nature run by the Prandtl number $\sigma$ (nature run: $\sigma_{0}=9$, model: $\sigma_{\mathrm{A}}=15$ ). To evaluate $q$ and its sensitivities three Lorenz 1963 model experiments were carried out (Table 8.1).

\subsubsection{Results}

Within the first experiment the observation operator $H$ used within the assimilation cycle was set as it is assumed within the derivation of $q$, i.e. $H=H_{0}=(1,0,0)$. The observation error $R$ of the assimilation cycle was set as constant $R \equiv R_{0}=0.15$ and equal to the observation error of the computation of the score $q$ (Table 8.1). The "butterfly"-trajectories generated 


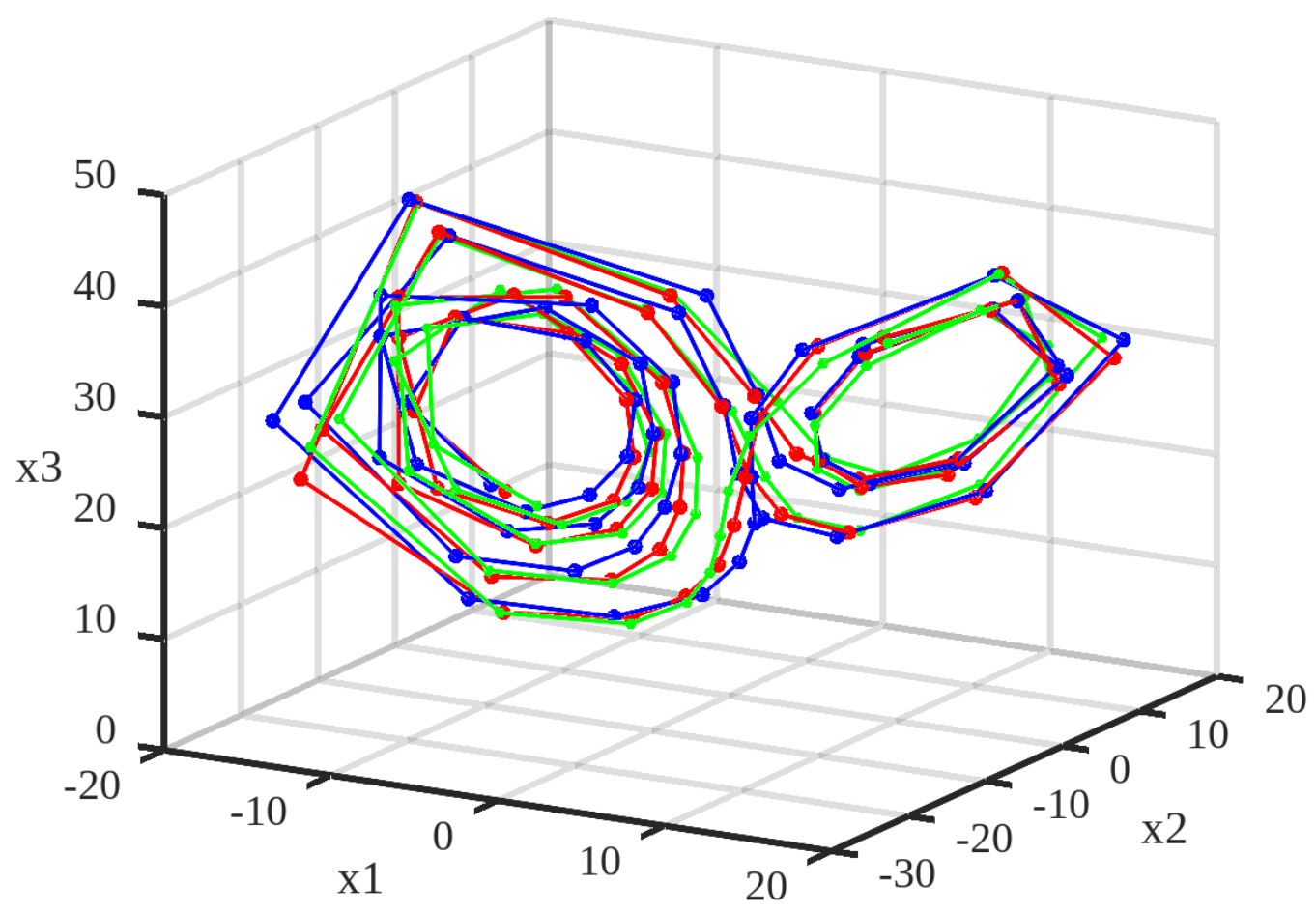

Figure 8.2: Nature run (green), background (blue) and analysis (red) are simulated by the Lorenz 1963 system for $N_{\text {cycle }}=500$ time steps. Shown are the first 60 time steps. At each time step an observation (dots) is taken, an assimilation step based on the ensemble Kalman filter is carried out and $q$ is calculated.

by the Lorenz 1963 system of the nature run and the assimilation cycle are shown in Figure 8.2. The state of the system consists of three components, whereby $x_{1}$ is observed. Thus, the ensemble covariances between the observed component $x_{1}$ and the other components $x_{2}$ and $x_{3}$ are of interest. In a NWP model, for example, the observed quantity could be a temperature, while the further components are humidity, pressure or wind. The score $q$ is determined at each time step for $x_{2}$ and $x_{3}$. In the following $q_{2}$ describes the score of the ensemble consistency between $x_{1}$ and $x_{2}$ and $q_{3}$ describes the score based on the covariances between $x_{1}$ and $x_{3}$. Due to the choice of $H$ and $R$ within the assimilation cycle, it is expected that a positive $q$ would coincide with a successful analysis step (Equation 8.2 is fulfilled) and a negative $q$ would be associated with an unsuccessful analysis step (Equation 8.2 is not fulfilled). If this is the case, the score $q$ matches the result of the assimilation step, which is illustrated by Figure 8.3 . Hence, the performance of the analysis steps depends on the use of appropriate background errors and $q$ gives an insight in the appropriate representation of the ensemble covariances between $x_{1}$ and $x_{2}$ and, $x_{1}$ and $x_{3}$. In this test case of $N_{\text {cycle }}=500$ time steps, $q_{2}$ was positive in about $43 \%$ of the cases, i.e. in $44 \%$ of the analysis steps the ensemble covariances between the observed state $x_{1}$ and the second state component $x_{2}$ matched the nature run. In contrast, in about $52 \%$ cases $q_{3}$ was positive, 


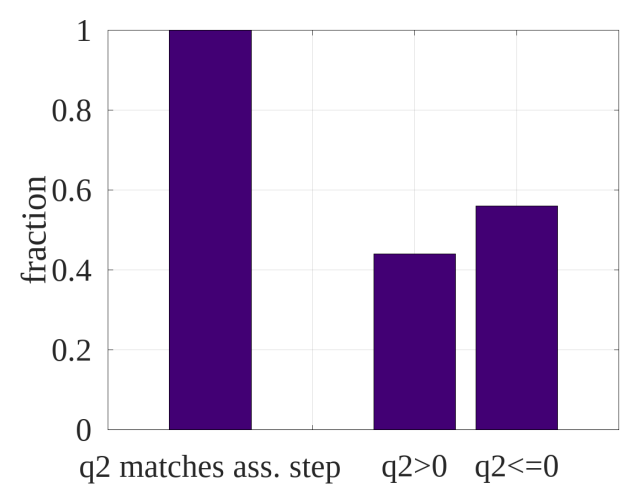

(a)

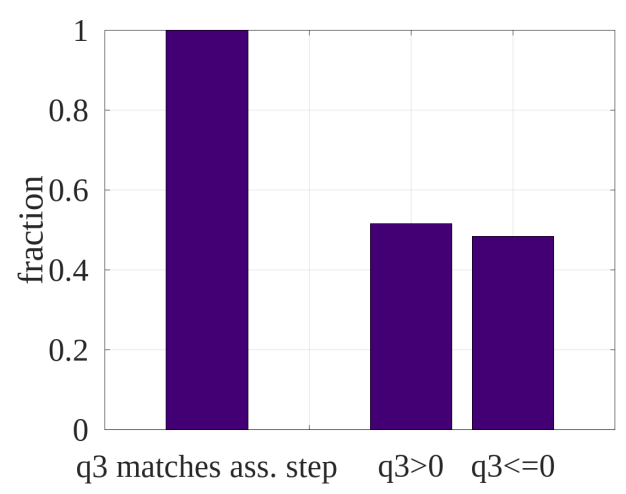

(b)

Figure 8.3: (a) Bar plot of the fraction of time steps in the experiment, that fulfill the conditions: sign of ensemble consistency score $q_{2}$ matches the impact of the assimilation step on $x_{2}, q_{2}>0$ and $q_{2}<=0$. (b) Like (a) but for the third component.

hence on average the assimilation of the observed $x_{1}$ has a positive impact on $x_{3}$.

Table 8.2: Contingency table of $q$ of the Lorenz 1963 system.

\begin{tabular}{|c|c|c|c|c|}
\hline & & & $q>0$ & $\mathbf{q}<=\mathbf{0}$ \\
\hline Eq. & 8.2 & fulfilled & $\begin{array}{l}\underline{\text { Hits: }} \\
q>0 \text { matches success- } \\
\text { ful analysis step }\end{array}$ & $\begin{array}{l}\frac{\text { Misses: }}{q<=0,} \text { but successful } \\
\text { analysis step }\end{array}$ \\
\hline Eq. & 8.2 & not fulfilled & $\begin{array}{l}\frac{\text { False Alarm: }}{q>0, \text { but unsuccessful }} \\
\text { analysis step }\end{array}$ & $\begin{array}{l}\text { Correct Negative: } \\
q<=0 \text { matches unsuc- } \\
\text { cessful analysis step }\end{array}$ \\
\hline
\end{tabular}

The first experiment was based on an analysis step which took the simplifications and the assumptions of the computation of the score $q$ (Section 8.1) into account. In the most cases the simplified observation operator $H_{0}=(1,0,0)$ is not applicable because the observation value is not covered directly by the model values or not directly located on the analysis grid. In general, the observation operator $H$ of the analysis step does not reproduce an exact image of the real observation. Here, due to the OSSE framework, the generation of the observation $y$ is clearly defined: $y$ is observed by the observation operator $H_{0}=(1,0,0)$ from the first model state $x_{1}$ of the nature run. As a first sensitivity test of the dependency of $q$ on the accuracy of the observation operator $H$ in the analysis step, several Lorenz 1963 experiments were performed with successively shifted observation operators, $H=(h, 0,0)$ with $h=\{0.80,0.81, \ldots, 1.19,1.20\}$ (Table 8.1). To limit the statistical noise of the results 500 time steps of each nature run and assimilation cycle were simulated.

The observation operator $H$ of the analysis step has mainly impact on the result of the assimilation step itself. Thereby, $H$ influences how often the sign 
of $q$ predicts the impact of the analysis step correctly (Figure 8.4). The sign of $q$ and the result of the analysis step match if the observation operator of the analysis step $H$ and the observation operator of the computation of the score $H_{0}$ are equal. Then every positive $q$-value is associated with a successful assimilation step and every negative $q$-value is associated with an unsuccessful assimilation step. With increasing difference between $H$ and $H_{0}$ the agreement between $q$-value and impact of the assimilation step decreases from $100 \%$ to $80 \%$ (Figure 8.4). This decrease occurs for $q_{2}$ and $q_{3}$. The decrease in the agreement is due to positive $q$-values associated with an unsuccessful analysis steps and vice versa. Therefore, the score $q$ is the more reliable, the more accurately the model equivalent represents the observations. The differences between the rat of hits, correct negatives, misses and false alarms of $q_{2}$ and $q_{3}$ are based on the model system an will likely differ in other systems.

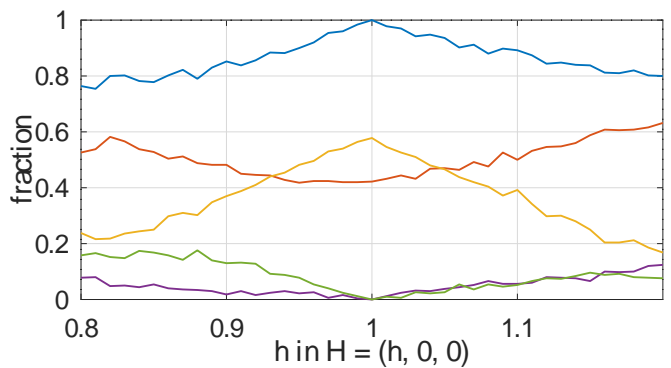

(a)

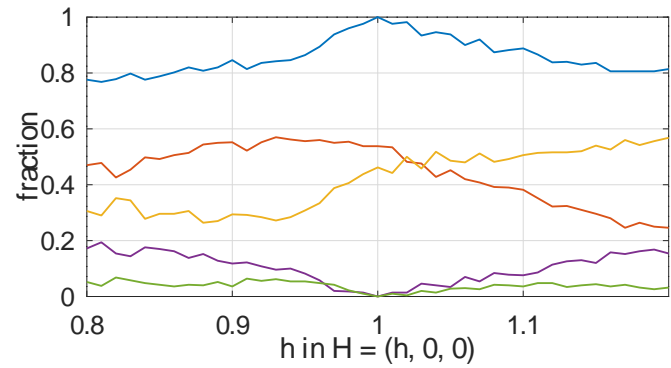

(b)

Figure 8.4: Observation operator $H$ sensitivity test of ensemble consistency score $q_{2}$ (a) and $q_{3}$ (b). The observation operator $H_{0}$ of the calculation of the $q$-values differs from the observation operator of the assimilation cycle $H=(h, 0,0)$. Several choices of $h=\{0.80,0.81, \ldots, 1.19,1.20\}$ are evaluated. Shown is the fraction of $q$ matches the impact of the analysis step (blue). Based on Table 8.2 hits (red), correct negatives (yellow), false alarms (purple), and misses (green) are shown.

In addition to the inaccuracy of the observation operator, an uncertainty of the observation error covariance matrix $R$ could also reduce the informative value of $q$. Within the third Lorenz 1963 experiment the observation error variance $R=\{0.01,0.02, \ldots, 0.29,0.30\}$ of the analysis step differs from $R_{0}=0.15$ which was assumed within the calculation of $q$ (Figure 8.5). If different $R$ values are used in the analysis step and the computation of $q$, the consistency of the ensemble covariance between observed value $x_{1}$ and the other variable $x_{2}$ and $x_{3}$ can be over- or underestimated. If $R<R_{0}$ more false alarms occur. On the other hand, if $R>R_{0}$ more misses occur. To avoid these inaccuracies a consistent value of $R$ is important. Overall, the observation error or noise of the observed value $R$ has less influence on the determination of the reliability of the score $q$ than the choice of the observation operator $H$. Especially, in the case $R>R_{0}$ the agreement between the score $q$ and the impact of the analysis step (Equation 8.2 is fulfilled) is only slightly reduced. 


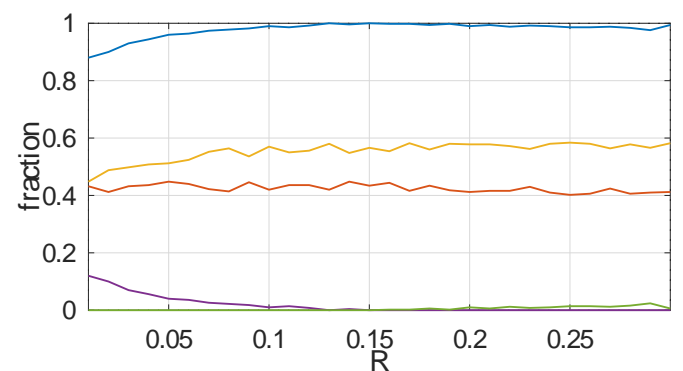

(a)

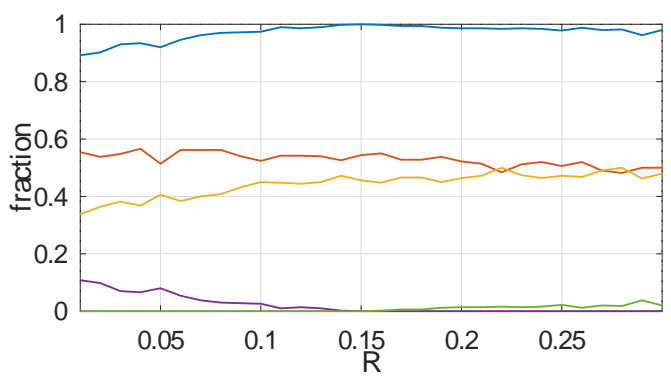

(b)

Figure 8.5: As in Figure 8.4, but as sensitivity test of the observation error covariance matrix $R$ with $R \equiv r=$ $\{0.01,0.02, \ldots, 0.29,0.30\}$ and $R_{0}=0.15$.

\subsubsection{Summary}

The score $q$ introduced in Section 8.1 is an estimator of the consistency of the ensemble covariances. The quality of its predictions will depend on the assimilation system it is deployed for. In this section, the score $q$ was tested using an assimilation system with a fully controllable forecast model: the Lorenz 1963 system. The focus was on the impact of observation operator $H$ and observation covariance matrix $R$ on the reliability of the score $q$.

With the simplification of $H_{0}=(1,0,0)$, model space and observation space are defined as identical. Through differences between $H_{0}$ and $H$, the ensemble covariances have to be translated from the model space into the observational space. Thus, the ensemble covariances in model and observational space differ from each other. This differences become visible once the sign of the score $q$ no longer matches exactly with the impact of the analysis step. The observation covariance errors $R$ also influence the reliability of $q$. The sign of $q$ is usable as an indicator for Equation 8.2 for being fulfilled or not, even if $R$ deviates from $R_{0}$. For this experiment, the noise of the observation has lower impact on the reliablity of $q$ than the choice of the observation operator. An even more precise evaluation of the sensitivity of the score $q$ to the observation error covariance $R$ would be possible by calculating $\frac{\delta q}{\delta R}$. Likewise, calculating $\frac{\delta q}{\delta H}$ would allow to estimate the local sensitivity of the score $q$ to the observation operator $H$.

\subsection{Experiments with Synthetic Land Surface Temperature}

Based on the results of Chapter 7, the covariances between the LST and near surface temperatures (soil and atmosphere), and the LST and near surface humidity (soil and atmosphere) are investigated. Similar to the soil moisture covariance study by Lin and Pu (2018), the correlations between LST and the top layer soil temperature $\left(T_{\mathrm{so}}(1)\right)$, LST and soil moisture $\left(W_{\mathrm{so}}(1)\right)$, LST and the bottom-layer (model level 50) atmospheric temperature $(T(50)$ ), and LST and specific humidity $(Q V(50))$ are evaluated. Hereby, each analysis grid point is evaluated independently without consideration of the neighboring pixels. In 
addition, the score $q$ is used to verify whether these correlations match the nature run. Thus, the following questions are answered within this section:

- What are the correlations between the near-surface variables and the land surface temperature?

- Do the model ensemble correlations of the assimilation cycle fit those in the nature run?

\subsubsection{Experimental Setup}

The synthetic setup of the LST OSSE experiment provides a known truth. That is why it is a good environment to test the score $q$ and the proper operation of the COSMO-KENDA system. The synthetic observations and their errors can be defined exactly (Chapter 6). Hence, it is possible to work with the simplified observation operator $H_{0}$ within the assimilation. To allow the usage of $H_{0}$ as observation operator in the analysis step, the observation operator $H$ of the LST was adjusted in this section. In the following experiments the synthetic observations are observed directly at the location of the COSMO-model grid points and the model equivalent $H\left(\overline{\mathbf{x}}^{\mathrm{b}}\right)$ is directly defined by the ensemble mean of the diagnostic variable $T_{\mathrm{g}}$. To examine the score $q$ (Equation 8.16) properly, both the daytime (1000 LT to 1400 LT) and the nighttime (2200 LT to 0200 LT) of the case studies in March and August 2017 are investigated.

In the current operational use, the COSMO-KENDA ensemble consists of 40 ensemble members and one deterministic run. To overcome restrictions due to the ensemble size KENDA offers several tools. The tools include the possibility to localize the observational impact and adjust covariances and model and observation errors:

- Adaptive observation error covariance matrix: Usually the observation error covariance matrix $R$ is assumed as a diagonal matrix with the variances on its diagonal. Additionally, $R$ can be adaptively updated due to observation type and height. With help of the Desroziers method (Desroziers et al., 2005) it is possible to create an error table, which than can be used for updates of $R$.

- Adaptive background error covariance matrix inflation: The background error covariance matrix $\mathbf{P}^{\mathrm{b}}$ is usually underestimated. The number of ensemble members is to small to sufficiently represent a correct model error. After each analysis step $\mathbf{P}^{\mathrm{b}}$ can be increased by multiplication with $\rho$. The default value is $\rho=1.1$.

- Relaxation of Prior Perturbations: To increase the ensemble spread again after each assimilation step, the analysis perturbation matrix $W^{\mathrm{a}}$ is updated in ensemble space. That means that $\alpha_{p} I$ with $\alpha_{p}=0.75$ is added to the analysis perturbation matrix $\left(W_{\text {update }}^{\mathrm{a}}=\left(1-\alpha_{p}\right) W^{\mathrm{a}}+\alpha_{p} I\right)$.

These adaptive modifications of the $B$ - and $R$-matrix are not reflected in the score $q$, therefore they are not used for the following experiments. Since no 
error table is generated for the LST retrieval, $R$ is not additionally updated and the tool of the adaptive observation error covariance matrix has no impact on the calculation of $q$. For simplicity, however the standard deviation of each synthetic LST retrieval is set to the fixed value $0.5 \mathrm{~K}$. The relaxation of prior perturbations has also no direct impact on the ensemble covariances and the calculation of score $q$ because only the ensemble spread of the analysis is modified, in contrast to the analysis mean, which is used to determine a successful analysis step (Equation 8.2). Hence, the adaptive background error covariance matrix inflation had to be adjusted so that the same ensemble covariances are used in the analysis step and in the calculation of $q$. For this purpose, $\rho=1$ is set in the following to omit the matrix inflation in the assimilation cycle.

Furthermore, it is crucial to also consider the vertical and horizontal localization of the observation error matrix $R$ because the localization of the observational impact is regulated via $R$ (Section 2.2 ). To ensure that only one observation at an analysis step influences the atmosphere and the soil around the observation a strong horizontal localization is needed. Hence, the horizontal localization length scale $h_{\mathrm{loc}}$ was set to a constant value of $700 \mathrm{~m}$ in the conducted experiments, i.e. the localization cut-off is at about $2.5 \mathrm{~km}$ and the horizontal impact of one observation is restricted to the model column of the grid point where the observation is located. At the same time the vertical impact by the assimilation of the LST retrieval on the correlated atmospheric and soil variables should not be attenuated. Thus, the vertical localization scale $v_{\text {loc }}$ is set to $10 \ln (p)$, which is synonymous with no vertical localization at all. Table 8.3 illustrates how the KENDA system has to be adjusted to fulfill the mentioned conditions.

Table 8.3: Namelist settings of KENDA changed due to ensemble consistency score test case.

\begin{tabular}{|l|l|l|l|}
\hline Name & OSSE & Experiment & Explanation \\
\hline \hline rho & 1.1 & 1.0 & no extra adjustment of B \\
\hline $\mathrm{h}_{\text {loc }}$ & $5.5 \mathrm{~km}$ & $0.7 \mathrm{~km}$ & strong horiz. localization \\
\hline vloc & 0.3 & 10 & no vertical localization \\
\hline q_bound & True & False & no humidity adjustment \\
\hline rf & 3 & 1 & no coarse analysis grid \\
\hline sat_ad & True & False & no humidity adjustment \\
\hline hyd_bal & True & False & no pressure adjustment \\
\hline adap_loc & True & False & no adaptive localization \\
\hline
\end{tabular}

To save computational costs, the operational KENDA-system calculates the transformation matrices on a three times coarser grid. To avoid discrepancies between the $H$ used within the KENDA assimilation cycle and $H_{0}$ which is used to calculate $q$ within this section, the transformation matrices are calculated on the full COSMO-model grid of the experimental domain. Thus, the LST observation $y$ is derived by the interpolation described in Chapter 6 from the nature run on the COSMO-DE model grid of the experimental domain. The first guess $\bar{x}_{1}^{b}$ associated with the observed LST is the land surface temperature 
of the model forecast $\left(T_{\mathrm{g}}\right)$ at the respective grid point. Under consideration of these conditions $q$ can be derived in agreement with the assimilation cycle:

$$
\begin{aligned}
q_{1} & =\frac{\left(x_{2}^{\text {true }}-\bar{x}_{2}^{\mathrm{b}}\right) b_{21}\left(\left(y-\bar{x}_{1}^{\mathrm{b}}\right)\right.}{0.25+b_{11}} \\
q_{\text {norm }} & =\frac{q_{1}}{\left(x_{2}^{\text {true }}-\bar{x}_{2}^{\mathrm{b}}\right)^{2}} \\
q & =1-\left(q_{\text {norm }}-1\right)^{2} .
\end{aligned}
$$

Here, $x_{2}^{\text {true }}$ is assumed as the true state of one of the examined atmospheric or soil variables $\left(x_{2}^{\text {true }}=\left\{T(50), Q V(50), T_{\text {so }}(1), W_{\text {so }}(1)\right\}\right)$. Due to the adaptions mentioned above, the success of the assimilation cycle depends significantly on the quality of the $\mathbf{P}^{\mathrm{b}}$ matrix. Thus, the score $q$ provides information about the quality of the ensemble covariances and about the positive or negative impact of an analysis step. In this context $q>0$ coincides with a successful analysis step and $q<=0$ coincides with an unsuccessful analysis step (Figure 8.6).

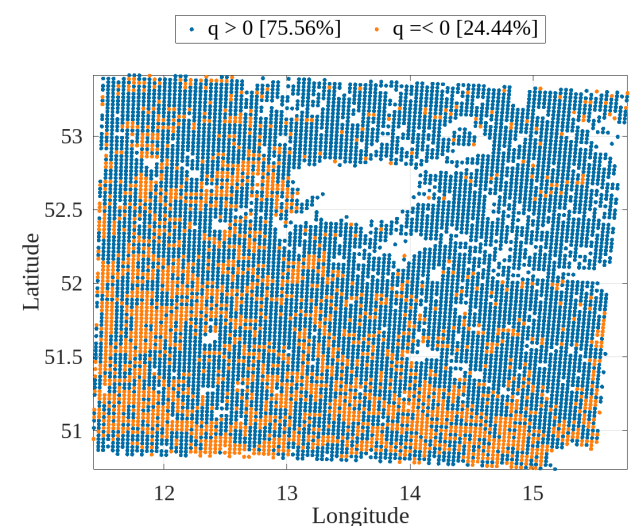

(a)

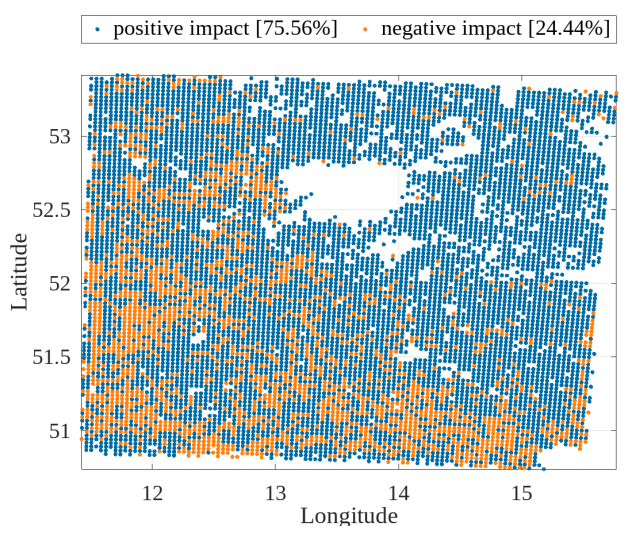

(b)

Figure 8.6: (a) Example of ensemble consistency score $q$ of LST and $T$ (50) on March 27, 2017 at 1200 UTC split into positive $q$-values (blue dots) and negative $q$-values (orange dots). The $q$-values are in agreement with (b) the impact of LST assimilation on $T(50)$ at the same time, a positive impact by the assimilation of LST is illustrated by blue dots and no or a negative impact by orange dots.

\subsubsection{Results}

To evaluate the ensemble covariances of the case studies from March and August 2017, adapted observations were generated, based on the conditions described in Section 8.3.1. The adapted LST observations were assimilated each hour initialized from EXPatso during the day (1000 LT to $1400 \mathrm{LT}$ ) and night hours (2200 LT to $0200 \mathrm{LT}$ ). In the following, the ensemble correlations between the LST and the near-surface variables $\left(T(50), Q V(50), T_{\mathrm{so}}(1)\right.$ and $\left.W_{\mathrm{so}}(1)\right)$ are assessed during night and day. Hereby, the score $q$ is used to determine how well these correlations reflect the nature run. Like in the assimilation experiments (Chapter 7), a boundary of ten grid boxes is omitted in the following evaluation 

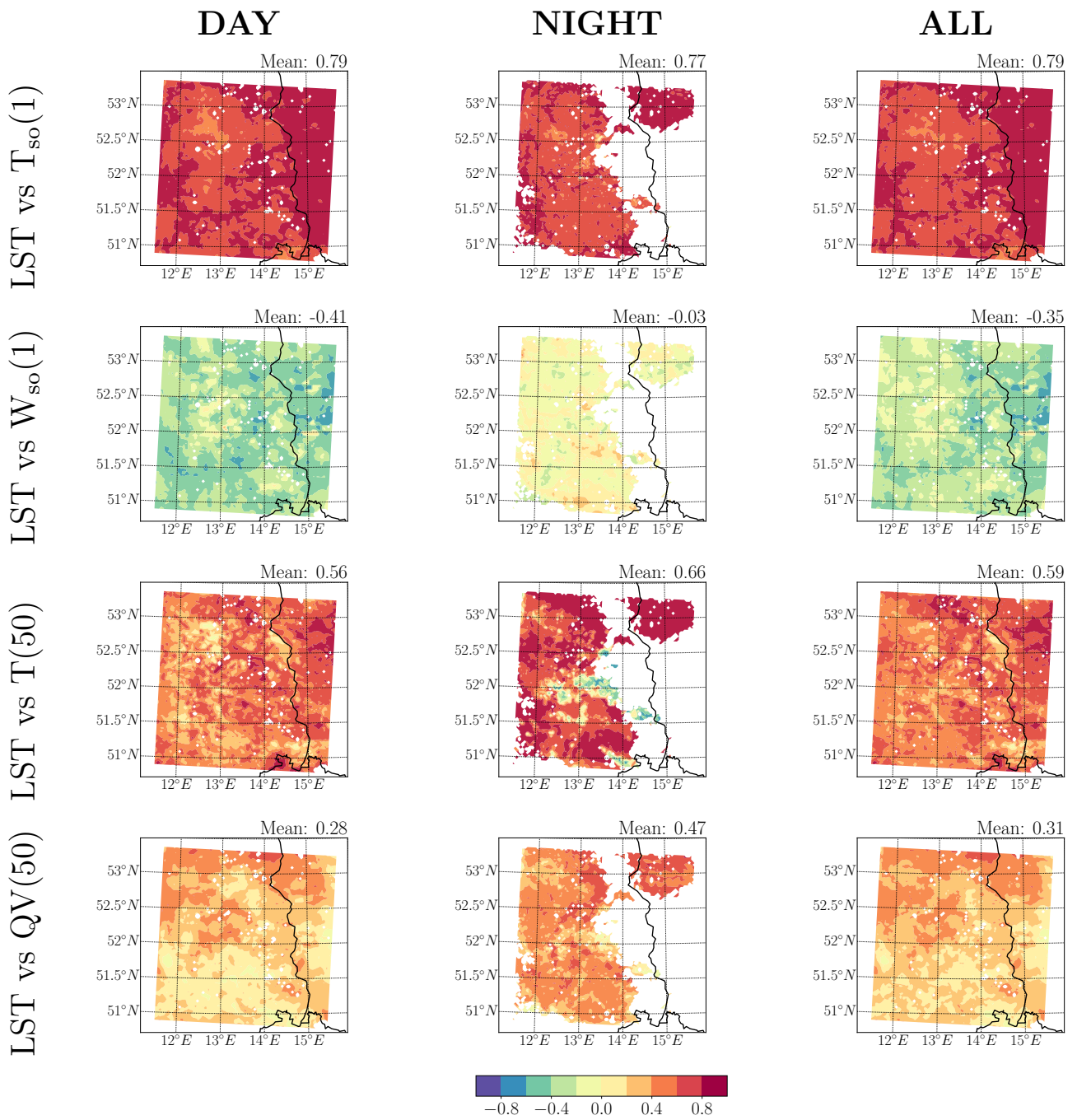

Figure 8.7: The temporally averaged ensemble correlations between LST and the top-layer soil temperature $T_{\mathrm{so}}(1)$ (first row), toplayer soil moisture $W_{\text {so }}(1)$ (second row), bottom-layer atmospheric temperature $T(5)$ (third row) and bottom-layer atmospheric specific humidity $Q V(50)$ (fourth row) from March 27 to March 29, 2017. Under the category 'DAY' all samples valid from 1000 LT to 1400 LT are summarized. Category 'NIGHT' covers the times valid from 2200 LT to 0200 LT and category 'ALL' covers all samples. The areal mean of each sample is shown above the respective plot. White areas indicate regions without observations due to clouds or lakes during the investigated period. 

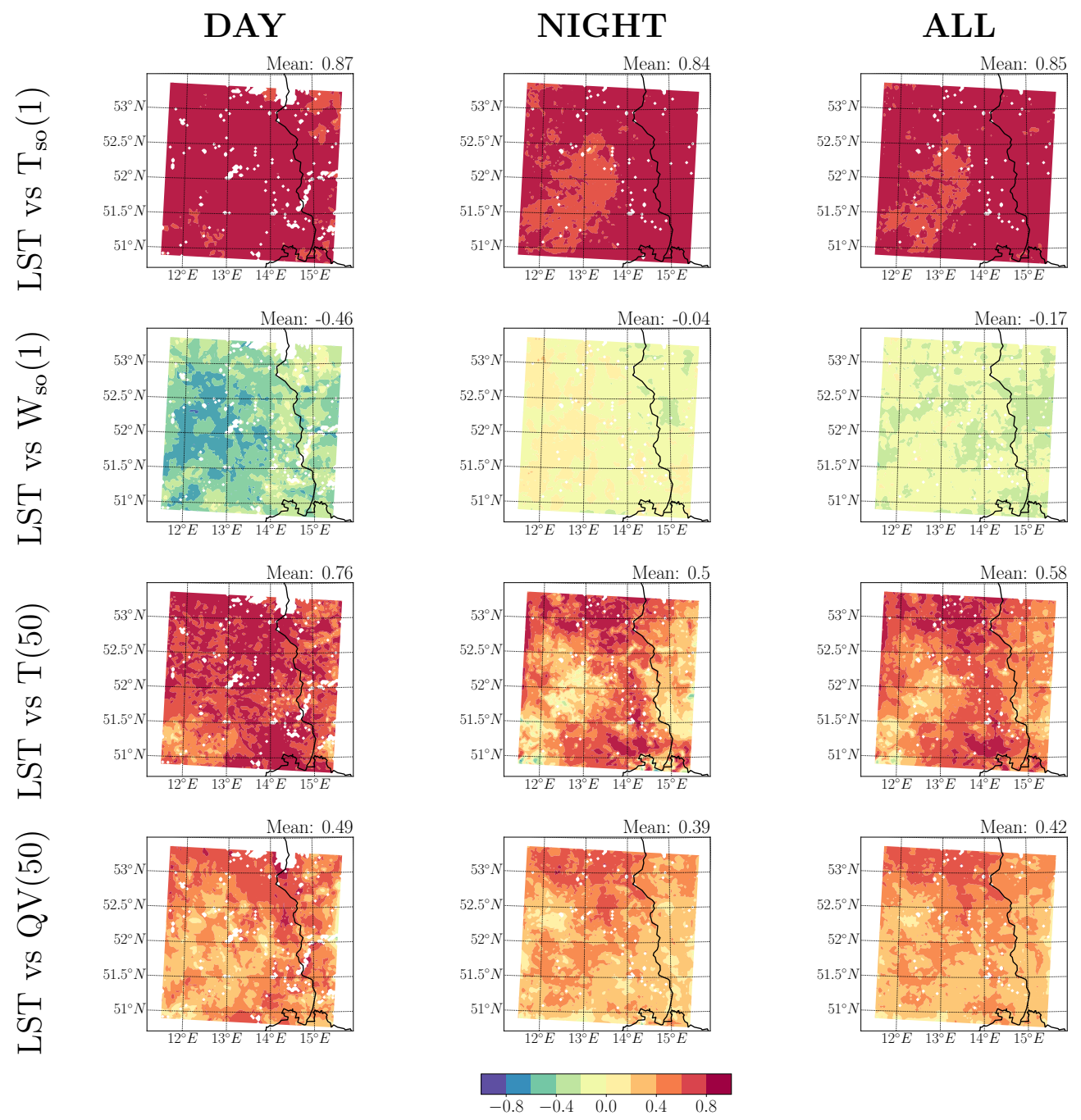

Figure 8.8: As Figure 8.7 but from August 28 to August 30, 2017.

to avoid boundary effects.

The correlation between LST and $T_{\text {so }}(1)$ is particularly strong compared to the other assessed near-surface variables (Figure 8.7 and 8.8, first rows). During the March and the August case study, the two temperatures are positively correlated with negligible differences between day and night and with around $8 \%$ higher correlation coefficient in August. The most noticeable difference between day and night are shown by the correlation between LST and $W_{\text {so }}(1)$ (Figure 8.7 and 8.8, second rows). In both cases, March and August, the correlation is negative during the day, while at night the correlation coefficient is close to zero. During summertime the radiative solar heating is larger than during wintertime. Hence, the exchange between the soil and the atmosphere due to turbulent fluxes is stronger during summertime. The stronger correlation between LST and the bottom-layer atmospheric variables, $T(50)$ and $Q V(50)$, in summer is also evident in the conducted case studies of this thesis (Figure 8.7 and 8.8, third and fourth rows). Both considered atmospheric variables are positively correlated with the LST whereby the temperature $T(50)$ is more strongly correlated than the specific humidity $Q V(50)$. While in March the correlation of 
LST with the atmospheric variables is stronger at night, in August it is stronger during the day.

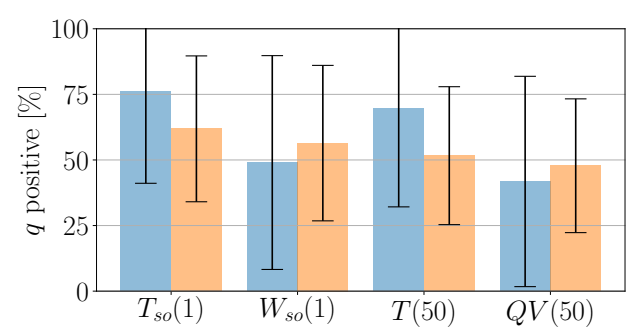

(a)

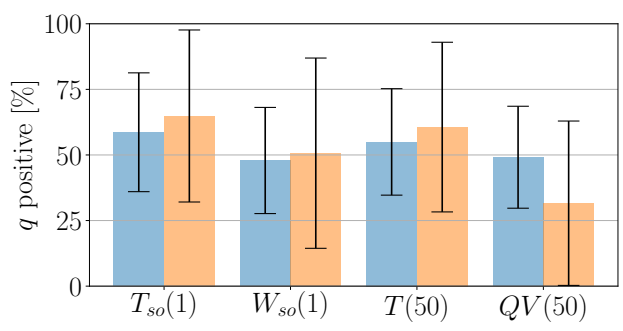

(b)

Figure 8.9: Evaluation of appropriate correlations of the case studies in (a) March and (b) August, 2017. Shown is the percentage of $q>0$ computed for the ensemble covariances between LST and surface-near variables such as bottom-layer atmospheric temperature $(T(50))$ and specific humidity $(Q V(50))$, and top-layer soil temperature $\left(T_{\mathrm{so}}(1)\right)$ and moisture $\left(W_{\mathrm{so}}(1)\right)$ shown during night (blue bars) and during day (orange bars). The black error-bars indicate the standard deviation.

Improvements due to the assimilation of LST were mainly achieved for soil and atmosphere temperatures. Here, also the correlation with the observation is strongest. The next step is to check how well the score $q$ classifies the found correlations and whether they fit the nature run. With regard to the temperatures, not only the correlation with LST is more pronounced, but they also match better with the nature run than the bottom-layer atmospheric specific humidity and the top-layer soil moisture (Figure 8.9). In March during night and in August during day, when the temperatures are strongly correlated to the LST, the score $q$ is mainly positive as well. The weaker correlation between the LST and the humidity variables is accompanied by a score $q$ that is frequently below zero. In most of the investigated cases, the probability for the humidity variables of a positive $q$ is about $50 \%$. Only during daytime in August the atmospheric specific humidity is noticeable. Here, the correlations determined by the ensemble only match the nature run in $30 \%$ of the cases.

At certain points in the case studies the assimilation of LST had a particularly positive or negative impact on the atmosphere and soil - so we ask the question whether the score $q$ also fits or fits not, respectively. However, some examples follow in detail. The first example deals with the impact of LST on the atmospheric temperature in different height levels. In the experiment during August, the RMSE of atmospheric temperature was improved during day, but during the second night, the RMSE of the atmospheric temperature increased above the ABL (Figure 7.17a). To compare the different height levels, the atmospheric temperature of the bottom-layer $(T(50))$ and of one layer near the top of the ABL during day $(T(42))$ are examined for their $q$-values (Figure 8.10). During daytime, the $q$-values of $T(50)$ and $T(42)$ are similar and positive in around $65 \%$ of the cases. In contrast, during nighttime both temperature layers 

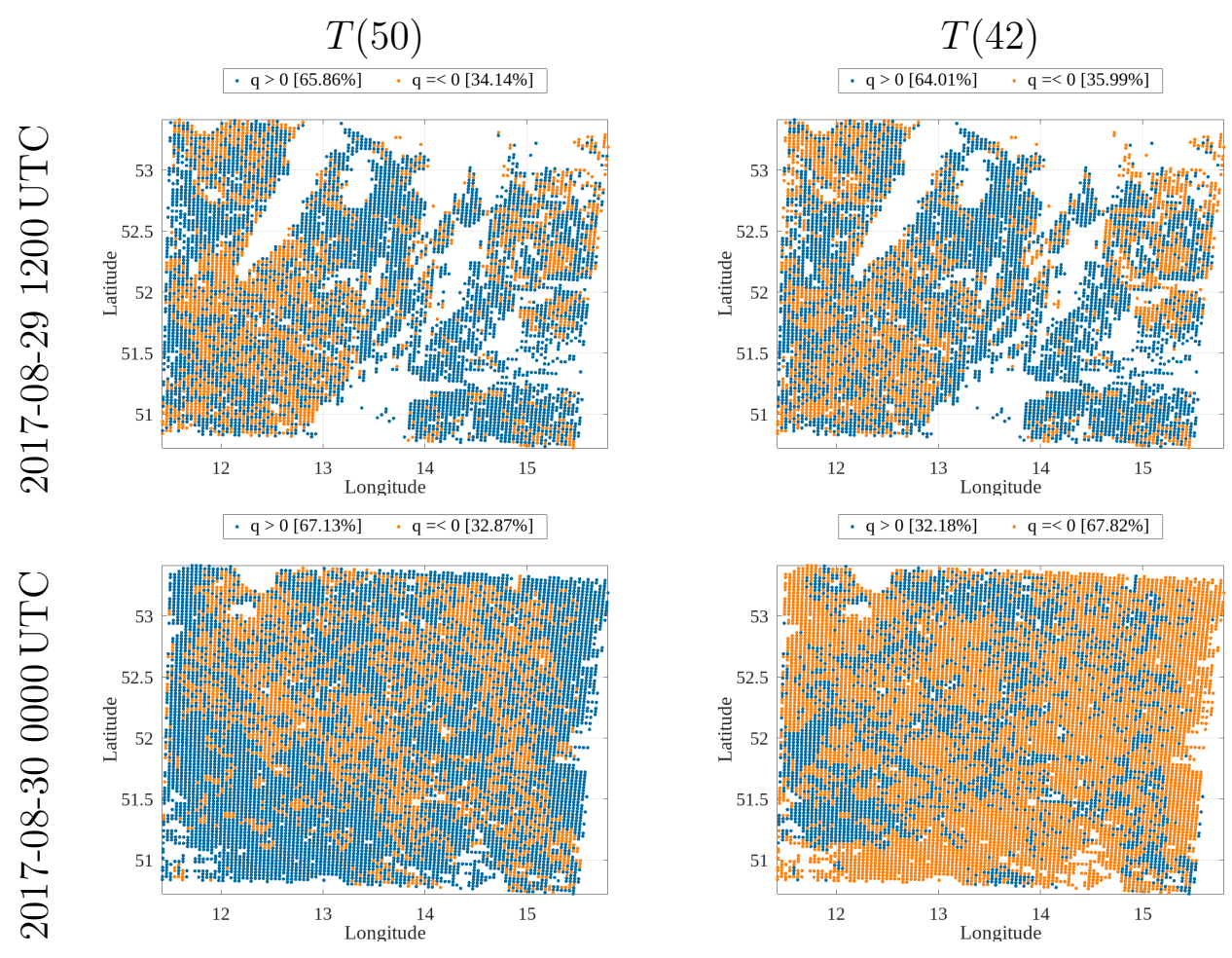

Figure 8.10: Maps of positive $q$ - (blue dots) and negative $q$-scores (orange dots). Shown are the $q$-values computed on the base of the ensemble covariances between LST and T(50) (first column), and LST and $T(42)$ (second column). The sign of the $q$-score is displayed at August 29, 20171200 UTC (first row) and at August 30, 20170000 UTC (second row). White areas indicate regions without observations due to clouds or lakes.

differ distinctly. While $67 \%$ of the $q$-values of the temperature in the bottomlayer are positive, only $30 \%$ of the $q$-values of $T(42)$ are positive, i.e. within the model layer above the nocturnal ABL, the ensemble of the assimilation cycle and the nature run do not match. This mismatch results in an analysis, which is pulled in the wrong direction. Hence, the assimilation of LST has a negative impact on $T(42)$.

Another interesting case in August concerns the impact of LST assimilation on specific humidity, the impact is negative on the first night of the case study and positive on the second night (Figure 7.19a). However, the fractions of positive and negative $q$-values at these times shows only a small difference. On the first night, only about $5 \%$ less $q$-values are positive. Thus, the impact of LST assimilation seems to be due to noise within the background error covariance matrix.

The influence of the assimilation of LST on soil moisture has a mainly positive impact during March (Figure 7.18b) and a mainly negative impact during August (Figure 7.15b). However, there is always a shorter time range when the influence of LST assimilation is exactly the opposite. If these points in time are compared, it can be seen that the positive impact coincides with 

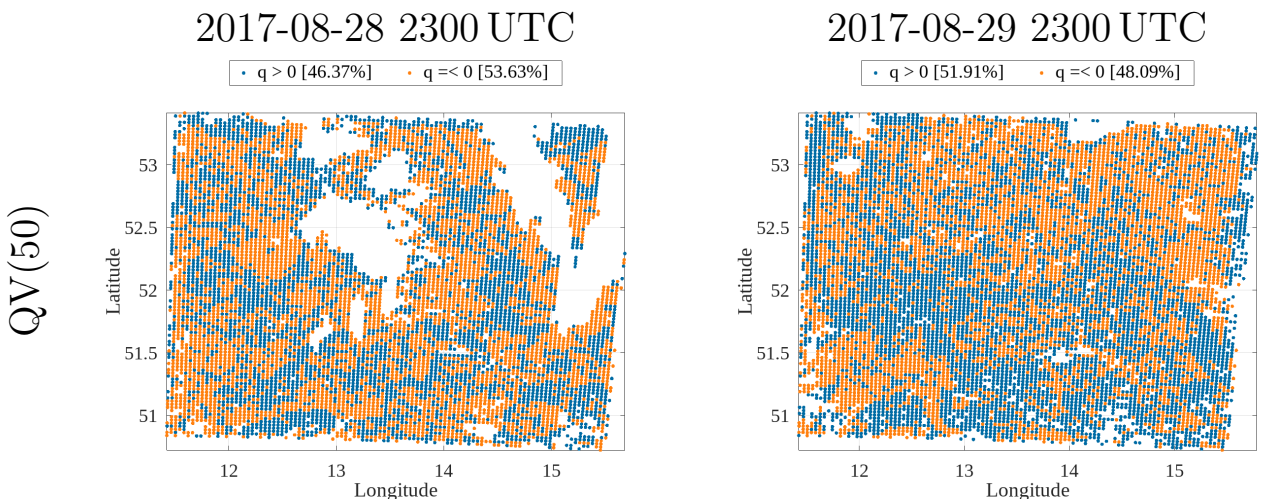

Figure 8.11: As Figure 8.10 but shown is the sign of the $q$-score computed on the base of the ensemble covariances between LST and bottom-layer atmospheric specific humidity $Q V(50)$. The sign of the $q$-score is shown at August 28, 20172300 UTC (first column) and at August 29, 20172300 UTC (second column).

(a) 2017-03-27 0600 UTC $\cdot \mathrm{q}>0[50.47 \%] \cdot \mathrm{q}=<0[49.53 \%]$

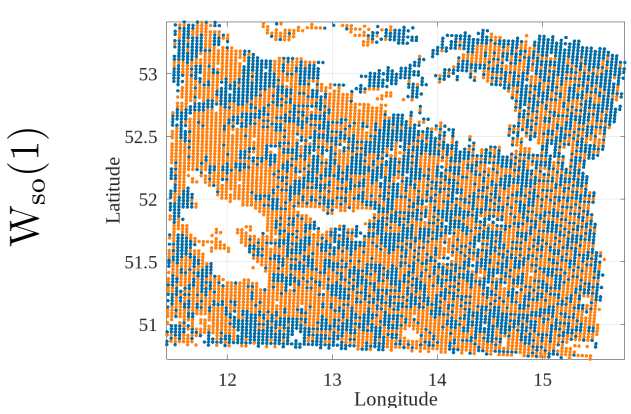

(c) 2017-08-28 1700 UTC $\cdot \mathrm{q}>0[54.44 \%] \cdot \mathrm{q}=<0[45.56 \%]$

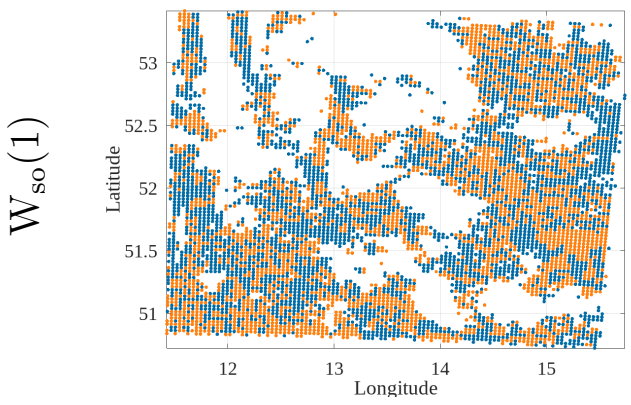

(b) 2017-03-27 1700 UTC $\cdot \mathrm{q}>0[62.33 \%] \cdot \mathrm{q}=<0[37.67 \%]$

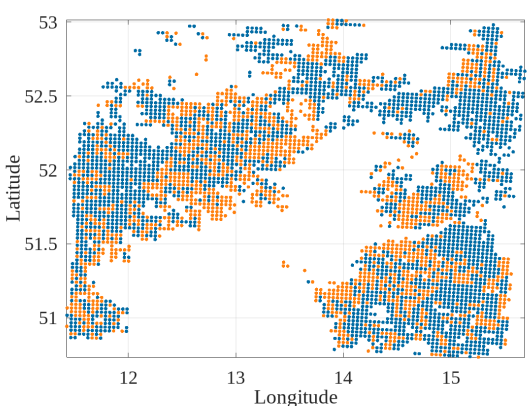

(d) 2017-08-29 1500 UTC $\cdot \mathrm{q}>0[46.16 \%] \cdot \mathrm{q}=<0[53.84 \%]$

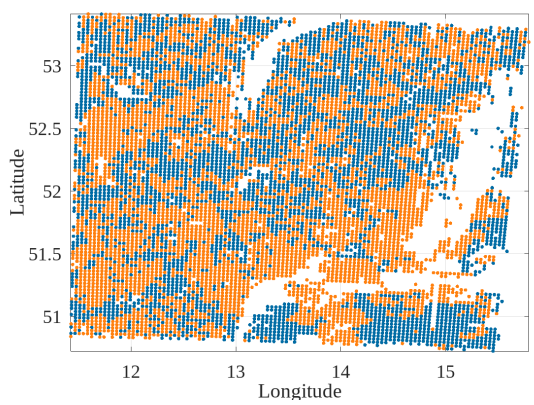

Figure 8.12: As Figure 8.10 but shown is the sign of $q$-scores computed on the base of the ensemble covariances between LST and top-layer soil moisture $W_{\mathrm{so}}(1)$. The $q$-score signs are shown at (a) March 27, 20170600 UTC, (b) at March 27, 20171700 UTC, (c) at August 28, 20171700 UTC and (d) at August 29, 2017 at 1500 UTC. 
less observations and a larger fraction of positive $q$-values in percentage terms (Figure 8.12). Thereby, in March $62 \% q$-values are positive and in August about $54 \%$.

\subsubsection{Summary}

The case studies in March and August 2017 were examined for their correlations and their $q$-values. It is found that the correlations between temperatures, especially between LST and soil temperature, are stronger than those between LST and the humidity and moisture variables. Particularly, in August at day and in March at night the background error covariances concerning the temperatures $\left(T(50)\right.$ and $\left.T_{\mathrm{so}}(1)\right)$ fit well to the nature run. The humidity variables $\left(Q V(50)\right.$ and $\left.W_{\text {so }}(1)\right)$ are less distinct. The impact on them by LST assimilation seems to be based more on noise than on correct correlations (Section 8.3.2). Since the nature run of this thesis corresponds to the higherresolution COSMO-model, one would expect more positive $q$-values due to the use of the same model during the assimilation cycle. If $q$ is below or equal to zero, the nature run does not match with the ensemble covariances of the lowerresolved COSMO-model. This supports the assumption that either in these case studies the ensemble spread is biased low or that the ensemble is not large enough to adequately represent the covariances. 


\section{Chapter 9}

\section{Conclusions}

LST retrievals have been assimilated in an OSSE framework in a coupled landatmosphere NWP-model. To obtain a suitable LST model equivalent, the representation of LST in the COSMO-model was evaluated and two vegetation temperature schemes were analyzed. For improvement of the model equivalent the skin conductivity scheme (Schulz and Vogel, 2020) was implemented and applied in the experiments. With the conducted OSSE experiments the potential impact of the assimilation of LST was determined and differently coupled land-atmosphere assimilation systems were tested. The experiments based on the weakly coupled data assimilation system were based on control vectors which either includes only atmospheric or soil variables. In the strongly coupled system, however, the control vector contained atmospheric and soil variables.

The experiments included single observation experiments to verify the functionality of the strongly coupled land-atmosphere assimilation system and two-day clear sky case studies in March and August 2017. Within the case studies LST was assimilated hourly and every six hours a 24 hour forecast was initialized from the assimilation cycle. The comparison of the weakly coupled and the strongly coupled assimilation system showed that the strongly coupled assimilation system reduced the RMSE of soil and atmospheric temperature stronger than the weakly coupled systems. These experiments were the first, that assimilated LST simultaneously for the land and the atmosphere within the COSMO-KENDA system. Thus, this assimilation approach is one step further than Candy et al. (2017) and Bosilovich et al. (2007), who assimilated LST into a land model, which was coupled with an atmospheric model. Lin and $\mathrm{Pu}(2019)$ compared a weakly and a strongly coupled assimilation system for the assimilation of soil moisture. They, as well, found that the fully coupled land-atmosphere assimilation system outperforms the weakly coupled one. The positive impact of LST assimilation on the first guess was larger during daytime, where a stronger coupling between LST and ABL temperature was found. The temperature forecasts of the ABL initialized from the assimilation cycle improved for up to nine hours and even more, if the soil temperature and moisture were part of the control vector. Further investigations of the covariances using a newly introduced ensemble consistency score $q$ showed that the covariances of the nature run were well reproduced for the temperatures within the soil and within the ABL. In contrast, the covariances between LST 
and the moisture variables of the nature run were often not well represented in the model ensemble. As a result, the influence of LST assimilation on temperature was beneficial, while it could also be detrimental to moisture.

\subsection{Discussion of Results}

To create a good starting point for the assimilation of LST, it was necessary to first answer the following questions:

- How to implement a vegetation/canopy temperature parameterization into the COSMO-model to predict a more accurate land surface temperature?

- Which implementation fits best with the LST retrieval derived from the remote sensing measurements to get a sufficient model equivalent?

The comparison between the LST retrieval derived by the Land-SAF and the LST of the COSMO-model showed that the amplitude of the modelled LST during the diurnal cycle was to small (Figure 5.5). Especially at night, the simulated temperatures at the land surface were too warm. The evaluation of the canopy scheme (Braden, 2012; Samuelsson et al., 2006) and the skin conductivity scheme (Viterbo and Beljaars, 1995; Verhoef and Vidale, 2012; Schulz and Vogel, 2020) showed that both schemes were able to reproduce the temperature amplitude during the day, but only the skin conductivity scheme was able to reproduce the colder LST retrievals at night (Figure 5.6). Hence, the skin conductivity scheme was employed into the nature run, to perform the experiments and, to derive the model equivalent and synthetic observations.

One main motivation of this thesis was to improve the prediction of the weather within the ABL by assimilating LST. Thus, to answer the question

- How does the assimilation of LST influence the atmospheric, the soil, and the land-atmosphere coupling values?

is an important part of this thesis. The conducted case studies showed that the first guess of the temperature of the soil and the ABL can benefit from the assimilation of LST. Hereby, the effect of LST assimilation on the atmosphere (Figure 7.16a and Figure 7.17a) depended on the diurnal cycle and the amount of available synthetic LST observations. The improvement due to the assimilation of LST increased with the growth of the mixed boundary layer and decreased with the transition to the nocturnal stable boundary layer. Above the ABL and during the evening transition, LST assimilation had also a negative impact. The combination of the smaller number of LST retrievals and the lower effect of LST assimilation on the second day of the March 2017 experiment indicate that the impact of LST assimilation is not observable for long in the atmosphere. In contrast, the near-surface soil layers could benefit over the whole experimental period. Here, the information of LST assimilation had a longer lasting positive impact on the soil that slowly permeates to deeper levels (Figure $7.16 \mathrm{~b}$ and Figure 7.17b). Whether the positive impact extends into the layers below $1 \mathrm{~m}$ depth can be answered by experimental periods longer than the ones employed here. Since the soil is more inert than the atmosphere, the propagation of 
information of LST assimilation into the depths is slower than the propagation through the atmosphere, thus the improvements in the soil layers also remained longer. This lead to the fact that the ABL still benefited from LST assimilation through the information in the soil even if the direct influence of LST assimilation on the atmosphere had already disappeared due to less observations, for example (Figure 7.16a). In both, the soil and the ABL, mainly the temperature predictions of the model benefited from the LST assimilation. The effects on moisture variables were mixed: the assimilation of LST could have a positive impact on atmospheric humidity in the afternoon, but a negative impact during the morning transition and night (Figure 7.18a and Figure 7.19a). The soil moisture could improve if additional moisture was introduced at an appropriate time (Figure 7.18b and Figure 7.19b). The turbulent fluxes of the land surface energy budget were not part of the control vector, so they were not directly updated in the analysis step. But due to changes in the temperature and the humidity of the atmosphere and the soil, the turbulent fluxes were adjusted as well. During daytime the impact of LST assimilation on the fluxes was clearly positive. The RMSE of the sensitive and the latent heat flux in March and August was reduced by up to $25 \%$. At night, however, the effect of the deterioration of atmospheric humidity on the latent heat flux was clearly visible.

The positive impact of LST assimilation on the temperature prediction remained visible in the atmosphere between the first three to nine forecast hours and in the soil over the entire forecast period of 24 hours. The improvement through LST assimilation in the prediction of the ABL temperature depended strongly on the available observations at the initial time of the 24 hour forecast, because the atmosphere does not keep the information over a long time period. If the LST retrieval was exhaustively available, the forecast of the atmospheric temperature could be improved by up to nine hours (Figure 7.25a and Figure 7.26a). Because the soil temperature varies on longer timescales than the atmospheric temperature, the soil kept the information of LST assimilation throughout the entire forecast. Due to the improved prediction of the soil temperature and moisture, the RMSE of the boundary layer temperature was partially reduced even after the first 9 hours of prediction. The better prediction of ABL temperature may potentially improve forecasts of ABL processes like convection, clouds, moist convection, and fog formation.

In order to assimilate LST to the best effect, different variants of the weakly and strongly coupled assimilation system were tested. In this process an answer for the research question

- Does the strongly coupled land-atmosphere assimilation system outperform the weakly coupled land-atmosphere assimilation system?

was found in the framework for COSMO-KENDA. The two-day experiments of March and August 2017 provided insight in the direct and indirect impact of LST assimilation within the weakly and fully coupled assimilation system. Two weakly coupled and one strongly coupled land-atmosphere assimilation systems were compared. In the first experiment, the control vector contained only atmospheric variables. Thus, the influence of LST assimilation on the soil was only 
indirect through the update of its upper boundary condition: the atmosphere. Therefore, the new information spread slowly in the soil and was correspondingly small. Hereby, the soil moisture gained almost no information from the assimilation of LST. In the second experiment, the control vector contained only the soil temperature and moisture. In this way just the soil was updated in the analysis step so that the influence on the atmosphere was indirectly given via the updated soil as its lower boundary condition. While the indirect influence of the atmosphere on the soil did not exhibit a clearly recognizable diurnal cycle, the soil influenced the atmospheric temperature mainly during the day (Figure 7.16a and Figure 7.17a). Thereby, the information transferred to the atmospheric humidity was rather low and the reduction of RMSE due to LST assimilation was below $10 \%$. In both experiments variables of the weakly coupled assimilation system that were directly coupled to the LST assimilation benefited more than the variables that were only indirectly coupled. In the third experiment, the control vector contained the atmospheric and the soil variables, so that both parts of the model system, land and atmosphere, were directly influenced by the assimilation of LST. The results show, that the impact of LST assimilation of the first and the second experiment were combined successfully. To conclude, the combination of a strong direct impact on the atmosphere and longer lasting indirect impact by the soil is promising. It was able to reduce near surface atmospheric temperature RMSE by up to $60 \%$ and the temperature of the upper soil levels by up to $40 \%$ within the OSSE framework. Near-surface observations, such as LST or soil moisture, can increase their influence on the overall system through the fully coupled land-atmosphere assimilation system, which can have a positive impact on the weather forecasts (Sgoff et al., 2020; Lin and $\mathrm{Pu}, 2020$ ).

The results of the three experiments in March and August 2017 lead to the presumption that the temperature-humidity correlations of the ensemble did not always correctly reflect the correlations of the nature runs. Hence, it was important to take a closer look at the background error covariances $\mathbf{P}^{\mathrm{b}}$ :

- How do background error covariances influence the impact of LST assimilation within the fully coupled land-atmosphere assimilation system?

Through the construction of the LETKF the impact of synthetic LST assimilation on the atmosphere and the soil was due to its background cross-correlations. In the conducted experiments, the correlation between the observations and the simulated atmospheric temperature depended on the time of day (Figure 7.4a and $7.5 \mathrm{a}$ and Figure 8.7 and 8.8). During the day, the LST and the temperature of the ABL were positively correlated because of the well mixed boundary layer. The correlation between LST and ABL temperature represented realistically the interaction between the land and the atmosphere due to turbulent fluxes. During the night, the correlation between LST and the temperature of the ABL was less clear. The correlation was partly negative, partly positive and also uncorrelated. This could be the result of the poorer representation of the stable boundary layer (Holtslag et al., 2013). Furthermore, the correlation between LST and soil moisture was significantly more pronounced during daytime (Figure 8.7 and Figure 8.8). The correlation between LST and soil temperature of the upper levels was always positively correlated, hence the soil temperature 
follows LST over the whole diurnal cycle. In contrast, the deeper soil layers did not change considerably within a day and were more or less uncorrelated with LST.

There are several possibilities to evaluate and adjust the background error covariances, which are, for example, reviewed by Bannister (2008a b) and Ehrendorfer (2007). Since the true state of the atmosphere is unknown, either the ensemble mean or time-shifted predictions are usually used to examine the background error covariances. This work was based on an OSSE, hence a true state was available to validate the consistency of the background error covariances with the correlations of the nature run. Thus the ensemble consistency score $q$ was introduced. The score $q$ indicates whether the background error covariances between the observation and a related model variable are consistent with the nature run $(q>0)$ or not $(q \leq 0)$. In the evaluation of $q$, it was noticeable that strongly correlated variables were more frequently associated with a positive score $q$, while weakly or uncorrelated ones were more frequently associated with a negative score $q$. Hence, the background error covariances of certain variables can be dominated by sample noise, which limits the positive impact of LST assimilation on moisture variables within this assimilation framework. The score $q$ proves to be a useful initial indicator of the ensemble consistency.

\subsection{Limitations and Outlook}

The period of the conducted experiments was limited to two-day periods. To further support the achieved results, besides the diurnal cycle also the seasonal cycle has to be explored and longer experiment have to be conducted. Furthermore the experiment domain should be extended. For example, Candy et al. (2017) found a benefit to forecasts of near-surface temperature due to LST assimilation, particularly over Africa.

Due to the assimilation of LST, particularly the temperature of the predicted ABL was improved. By the additional assimilation of near-surface moisture observations, the positive impact could be extended to further variables of the atmosphere, like the specific humidity. This is because the background error covariance matrix allows the information from the observations to influence each other and thus jointly influence the analysis (Baldauf et al., 2011). Synthetic studies by Han et al. (2013, joint assimilation of LST and microwave brightness temperature) and Abdolghafoorian and Farhadi (2019, joint assimilation of soil moisture and LST) and the study with real data by Tajfar et al. (2020a, joint assimilation of LST and reference-level temperature and relative humidity) showed promising steps in this direction.

A joint assimilation could also improve the background error covariances between the observed variables and the variables of the control vector. The effect on the humidity variables by the assimilation of LST, which in this thesis was probably mainly dominated by noise, could change positively if the joint assimilation provides a clearer signal within the background error covariances. 
One possibility to prevent spurious correlations between the near-surface observations and the atmosphere above the $A B L$ is a stricter vertical localization. In particular during night, this could reduce the negative impact of LST assimilation.

Overall the work of this thesis is restricted to the setup of an OSSE and an equal benefit of LST assimilation for operational systems with real data is not guaranteed. Hence, the experiments should be seen as a test of concept, as results from OSSEs are generally biased to better results than studies based on real observations. However, the results encourage the evaluation of LST assimilation within the coupled land-atmosphere system with real LST retrievals. The positive impact achieved by the assimilation of real LST retrievals into land models (Ghent et al., 2010) and weakly coupled land-atmosphere models (Candy et al., 2017) supports this future step. Our results show that assimilation of LST has a stronger impact on the lower atmosphere during the day than during the night. For example, Candy et al. (2017) has assimilated LST retrieval only at night, since the observation errors of the LST retrieval are higher during daytime. The results of this work suggest, that the observations should also be assimilated during daytime, to increase the impact of assimilation. Hence, a useful diurnal bias correction is necessary.

As also mentioned by Trigo et al. (2015), improved LST also has advantages for the use of further brightness temperature observations by satellites for assimilation. By improving LST, the simulation of brightness temperature can benefit as well and thus reduce the deviations between simulated and observed brightness temperatures. This would provide the opportunity to assimilate the information received by further surface and near-surface satellite observations into the numerical weather prediction models. These additional observations can improve the forecast of the processes in the ABL further. 


\section{Appendix A}

\section{Programming Lorenz 1963 System}

The programming of the Lorenz 1963 system is adopted from Chapter 6 of Nakamura and Potthast (2015) and adjusted to the ensemble consistency score $q$. Hence, main parts of the coding and structure follow the scripts of Nakamura and Potthast (2015) and are as well implemented in OCTAVE (Eaton et al., 2020).

The Lorenz 1963 system is a dynamical system of three coupled ordinary differential equations

$$
\begin{aligned}
\frac{d x_{1}}{d t} & =\sigma\left(x_{2}-x_{1}\right) \\
\frac{d x_{2}}{d t} & =x_{1}\left(\rho-x_{3}\right)-x_{2} \\
\frac{d x_{3}}{d t} & =x_{1} x_{2}-\beta x_{3}
\end{aligned}
$$

with $\sigma$ the Prandtl number, $\rho$ the Rayleigh number and, $\beta$ a non-dimensional wave number. The typical values of these three constants are: $\sigma=10, \rho=28$ and, $\beta=8 / 3$. In the performed experiments the constants $\rho$ and $\beta$ are fixed to the typical values, meanwhile for $\sigma$ in the nature run and the model different values are chosen. With the state vector of the system $\mathbf{x}=\left(x_{1}, x_{2}, x_{3}\right)^{T} \in \mathbb{R}^{3}$ the system can also be written as follows

$$
\begin{gathered}
\frac{d \mathbf{x}}{d t}(t)=F(t, \mathbf{x}) \quad \text { with } \quad \mathbf{x}(0)=\mathbf{x}_{\text {init }} \\
F(t, \mathbf{x}):=\left(\begin{array}{c}
\sigma\left(x_{2}-x_{1}\right) \\
x_{1}\left(\rho-x_{3}\right)-x_{2} \\
x_{1} x_{2}-\beta x_{3}
\end{array}\right) .
\end{gathered}
$$

The code of the forcing terms of the Lorenz 1963 dynamics is given as follows:

Code A.1 Function sim_Lorenz63_F to force the Lorenz 1963 dynamical system.

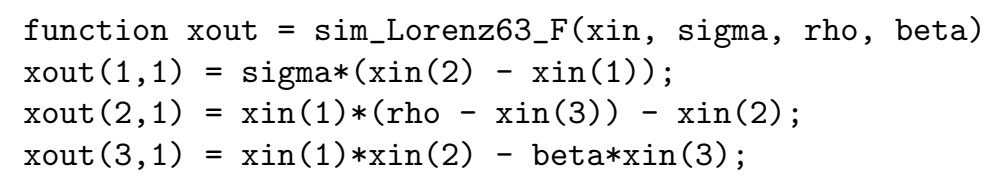


To numerically solve the coupled differential equations A.1 to A.3 a 4th-order Runge-Kutta scheme is used. It starts from an initial state $\mathbf{x}_{\text {init }}$ and iteratively calculates the future states

$$
\begin{gathered}
\mathbf{k}_{1}=F\left(t_{k}, \mathbf{x}_{k}\right) \\
\mathbf{k}_{2}=F\left(t_{k}+\frac{1}{2} h, \mathbf{x}_{k}+\frac{1}{2} h \mathbf{k}_{1}\right) \\
\mathbf{k}_{3}=F\left(t_{k}+\frac{1}{2} h, \mathbf{x}_{k}+\frac{1}{2} h \mathbf{k}_{2}\right) \\
\mathbf{k}_{4}=F\left(t_{k}+h, \mathbf{x}_{k}+h \mathbf{k}_{3}\right) \\
\mathbf{x}_{k+1}=\mathbf{x}_{k}+\frac{1}{6} h\left(\mathbf{k}_{1}+2 \mathbf{k}_{2}+2 \mathbf{k}_{3}+\mathbf{k}_{4}\right)
\end{gathered}
$$

with $h$ as time spacing of the iterations. The implementation in OCTAVE is given by

CoDE A.2 Function sim_Lorenz63_RungeKutta to numerically solve the ordinary differential equations of the Lorenz 1963 system by a 4th-order RungeKutta scheme.

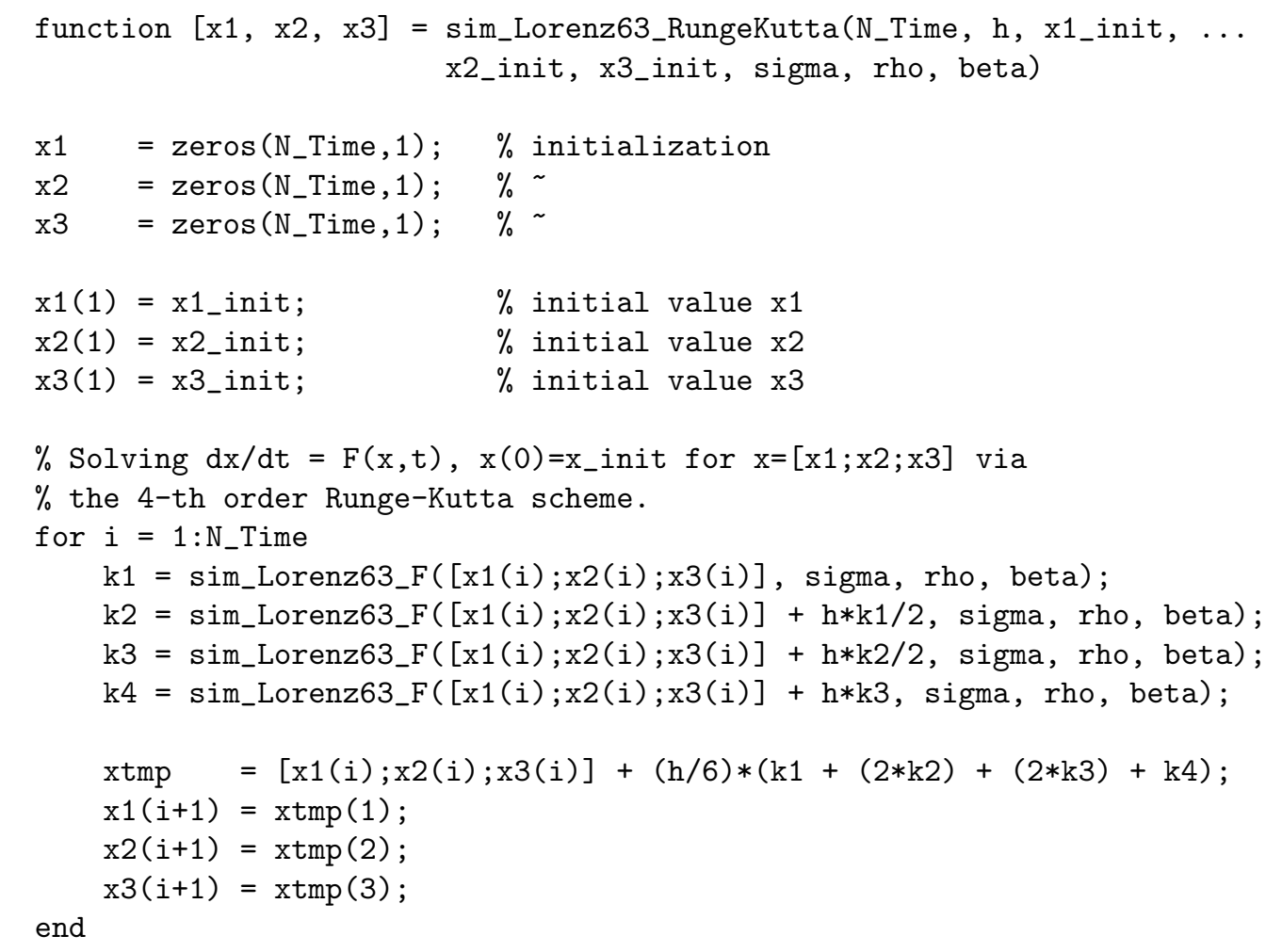

and includes the call of the function sim_Lorenz63_F.

The function sim_Lorenz63_Run gives the framework to generate the Lorenz 1963 dynamics. By calling this function an initial state or an ensemble of states with $L \in \mathbb{N}$ members is moved one time step into the future.

Code A.3 By function sim_Lorenz63_Run the Lorenz 1963 dynamics are generated. As input an initial state or an ensemble of states, a time step and a constant $\sigma$ are needed. 


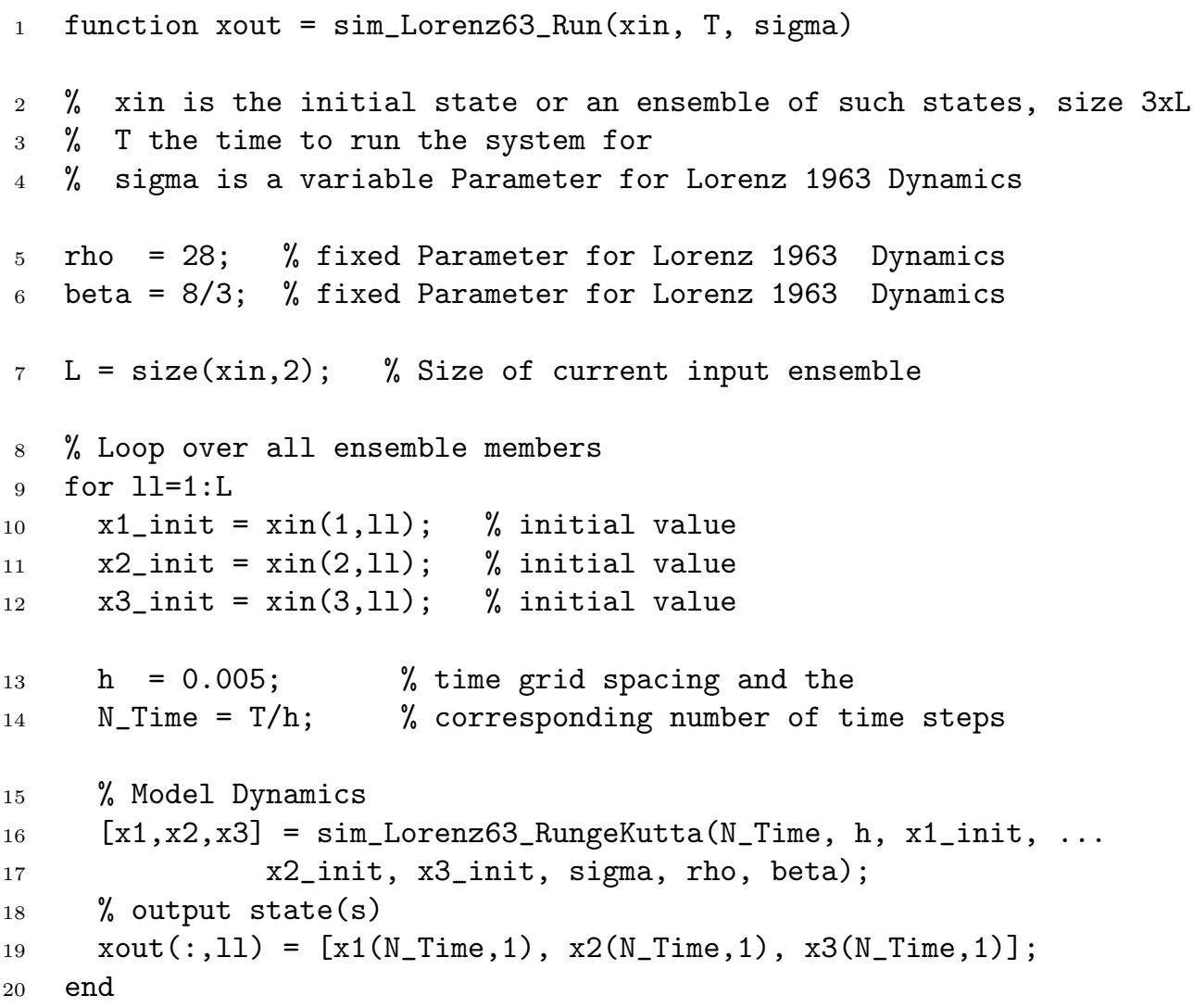

Based on the presented functions the OCTAVE script CodeA.4 produces the nature run and the measurement data of the first component of the state. By repeatedly executing the OCTAVE Function sim_Lorenz63_Run (CodeA.3) the nature run is transported through time. The time step is set to $d t=0.1$. At each time step the first component of the model state is measured. The measurements are generated with the observation operator $H_{\mathrm{NR}}=(1,0,0)$. Additionally some noise is added to mimic the observational error.

CODE A.4 OCTAVE script Generate_Nature_Run_and_Observations generates the nature run and its observational data $y$.

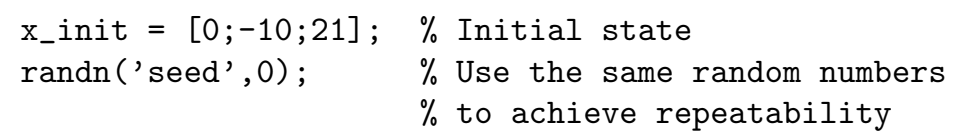

$\%$ 1) Setup Observation operator of "nature run"

$\%$ observing the first component of the state only

H_NR $=\left[\begin{array}{lll}1 & 0 & 0\end{array}\right]$;

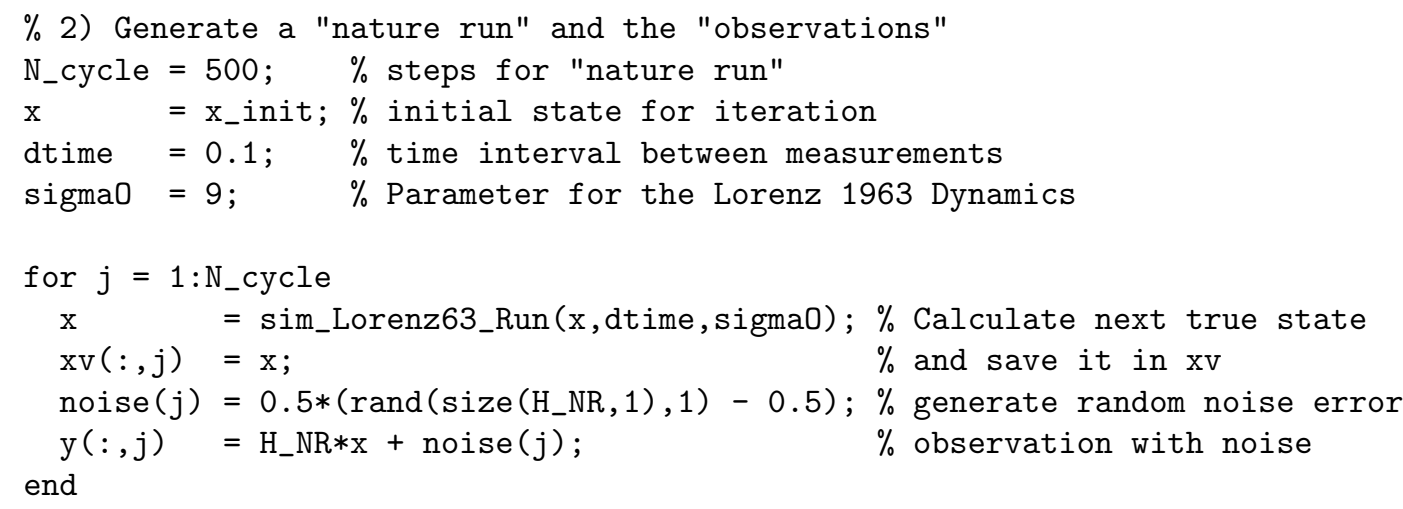


To avoid that the model error is underestimated the spread of the analysis ensemble is enlarged by a multiplication with the inflation factor 1.2 (CodeA.5. line 21).

Sensitivity tests were carried out to determine how strongly the informative value of the score $q$ depends on deviations between $H$ and $R$ in the analysis step and the $q$ calculation (Table 8.1). For the sensitivity test depending on $H$ this OCTAVE script was used:

Code A.6 Script to control sensitivity test of the dependency of $q$ on the accuracy of the observation operator $H$. Nature run, data assimilation cycle, computation, and evaluation of $q$ are executed by this script. The data is saved for subsequent analysis.

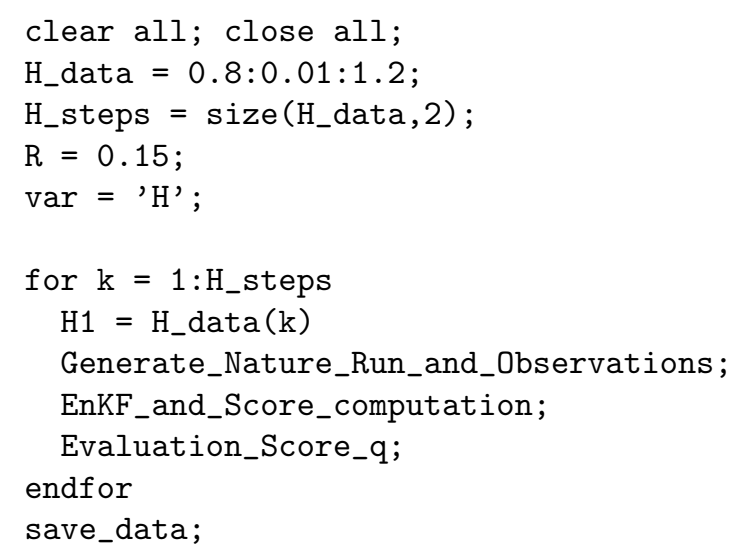

The sensitivity test depending on $R$ was performed by this OCTAVE script:

CODE A.7 Script to control sensitivity test of the dependency of $q$ on the accuracy of the observation error $R$. Nature run, data assimilation cycle, computation, and evaluation of $q$ are executed by this script. The data is saved for subsequent analysis.

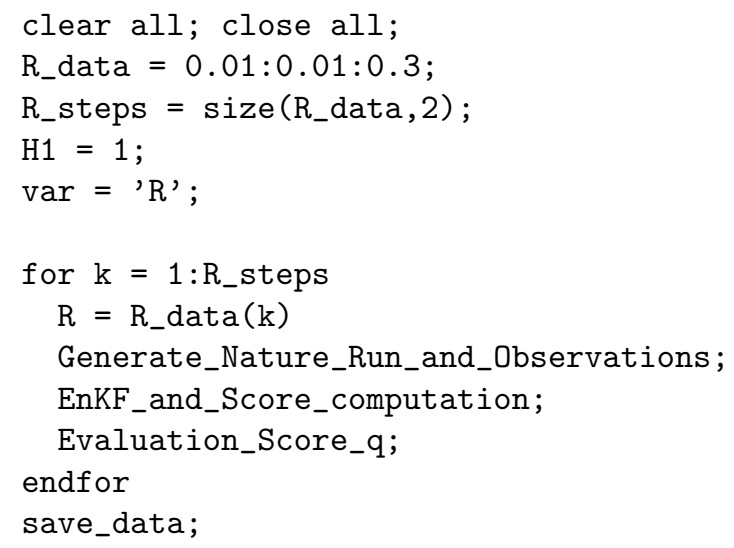

The quality of $q$ as an estimator of the assimilation success does not depend on $q$ being positive or negative, but on it correctly classifying one of either two cases: An successful assimilation step with Equation 8.2 fulfilled and an unsuccessful step vice versa. The evaluation is based on a contingency table, which groups the possible combined outcomes of score and assimilation step into four categories: hits, where $q>0$ coincides with a successful assimilation step, correct negatives, where $q \leq 0$ coincides with an unsuccessful assimilation step, misses, where $q \leq 0$ but the assimilation step is successful, and false alarms, where $q>0$ but the assimilation step is unsuccessful (Table 8.2). The OCTAVE script Evaluation_Score_q.m determines the fraction of these four categories: 
CoDE A.8 Analysis of score $q$ based on the contingency table (Table 8.2).

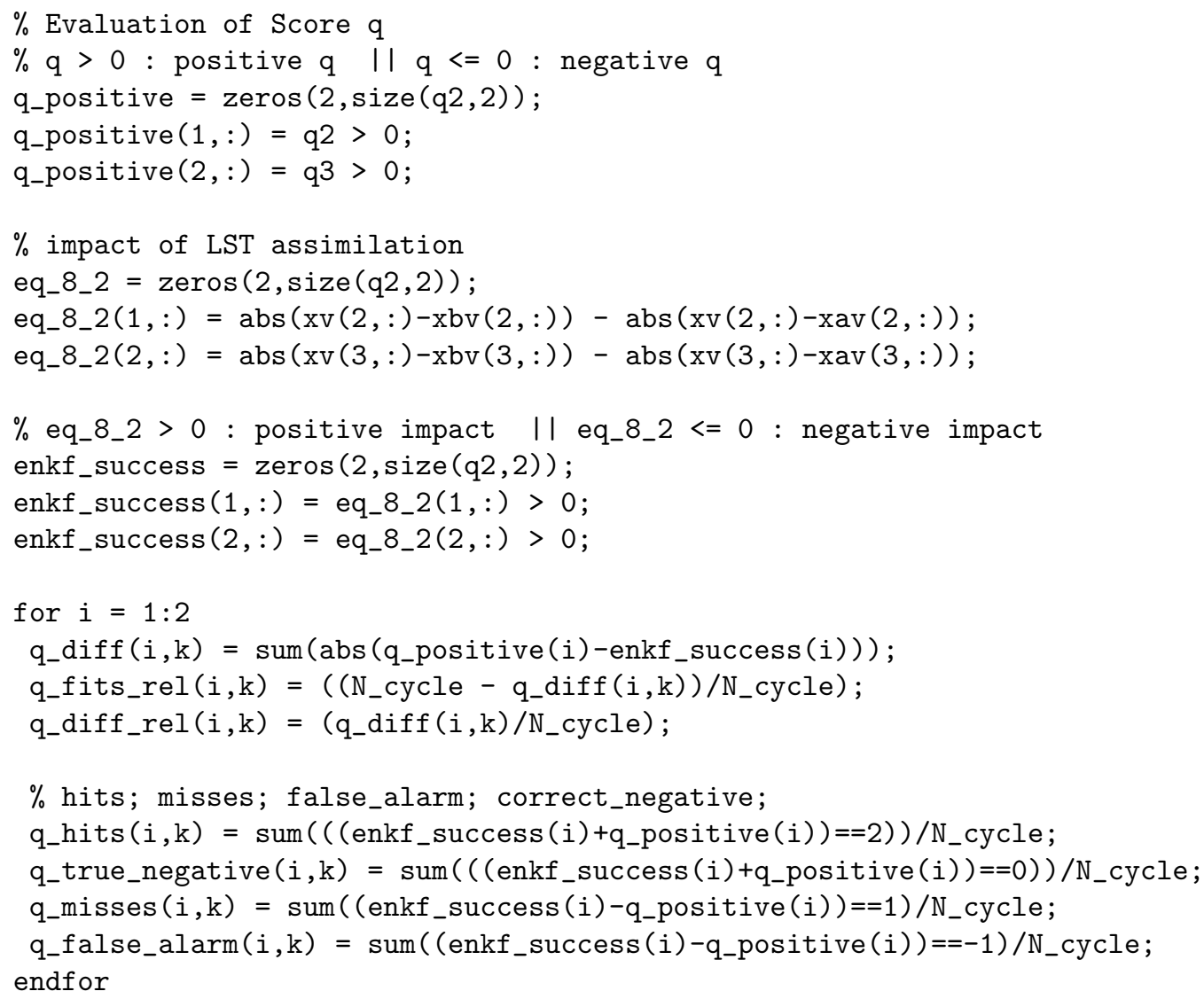

To save the data for subsequent analysis and visualization this OCTAVE script was used:

Code A.9 This script saves the data as .csv-file for subsequent analysis.

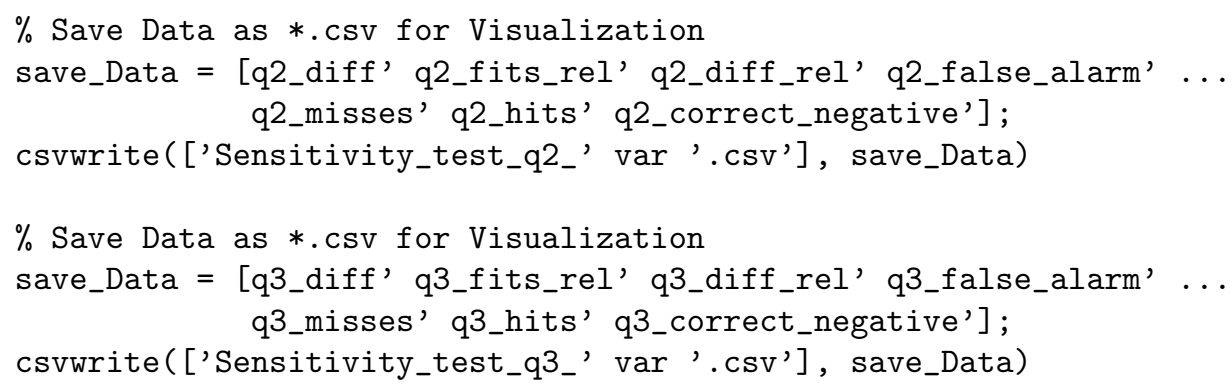




\section{List of Figures}

2.1 Schematic illustration of the Kalman filter work flow . . . . . . 13

2.2 Schematic illustration of used assimilation cycle work flow . . . 14

$2.3 \quad$ Evaluation of different localization-scales (soil) $\ldots \ldots . . . . .17$

3.1 Cloud cover of nature run . . . . . . . . . . . . . . . . . . . . . 19

3.2 Model surface height of nature run . . . . . . . . . . . . . 20

5.1 Comparison of LST retrieval and simulated LST . . . . . . . . . 25

5.2 Layer structure of soil temperature and energetic processes considered by TERRA_ML . . . . . . . . . . . . . . . . 27

5.3 Schematic illustration of canopy scheme with resistance approach 29

$5.4 \quad$ Schematic illustration of canopy scheme with skin conductivity approach . . . . . . . . . . . . . . . . . 32

5.5 Comparison of observed and simulated total cloud cover . . . . 33

5.6 Comparison of domain averaged observed and simulated LST. . 34

5.7 Distribution of deviations between observed LST and LST simulated by the different model implementations . . . . . . . . 35

5.8 Simulated LST minus observed LST against day time for all three model implementations . . . . . . . . . . . . . . . . 35

$6.1 \quad$ Example of synthetic LST retrieval . . . . . . . . . . . . . . . . 38

6.2 Distribution of the departures between LST of NR and observed LST, real and synthetic . . . . . . . . . . . . . . . 39

$7.1 \quad$ Distribution of single observations of LST . . . . . . . . . . . . 41

7.2 Distribution of observation minus first guess of LST combined with impact of one assimilation step on the atmospheric temperature of the lowest model level . . . . . . . . . . . . . 42

7.3 Cross sections of atmospheric and soil temperature innovation . 43

7.4 Profiles of soil and atmospheric temperature properties related to the assimilation LST (day) $\ldots \ldots \ldots$. . . . . . . . . . . . . 44

7.5 Profiles of soil and atmospheric temperature properties related to

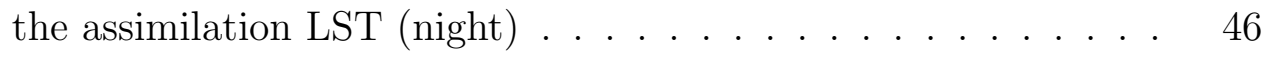

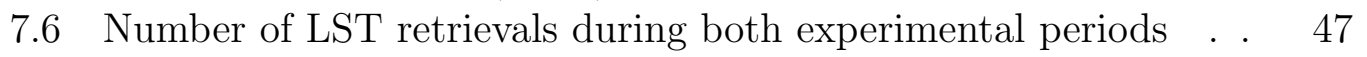

7.7 Observation minus model departure histograms . . . . . . . . . 49

7.8 Mean error evolution of boundary layer temperature and of soil temperature (March 2017) . . . . . . . . . . . . . 50

7.9 Mean error evolution of boundary layer temperature and of soil temperature (August 2017) . . . . . . . . . . . . . 50 
7.10 Mean error evolution of specific humidity of boundary layer and of soil moisture $($ March 2017) $\ldots$. . . . . . . . . . . . . 51

7.11 Mean error evolution of specific humidity of boundary layer and of soil moisture (August 2017) $\ldots \ldots \ldots \ldots \ldots$. . . . . . . . . 52

7.12 RMSE evolution of boundary layer temperature and of soil

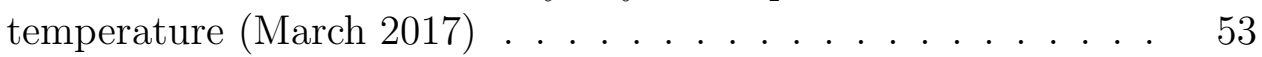

7.13 RMSE evolution of boundary layer temperature and of soil temperature (August 2017)] . . . . . . . . . . . . 53

7.14 RMSE evolution of boundary layer specific humidity and of soil moisture (March 2017) . . . . . . . . . . . . . . . 54

7.15 RMSE evolution of boundary layer specific humidity and of soil moisture (August 2017) $\ldots \ldots \ldots \ldots \ldots \ldots$

7.16 Evolution of relative change of RMSE boundary layer temperature and of soil temperature (March 2017) $\ldots \ldots$. . . . . . 56

7.17 Evolution of relative change of RMSE boundary layer temperature and of soil temperature (August 2017) . . . . . . . . . 56

7.18 Evolution of relative change of RMSE boundary layer humidity and of soil moisture (March 2017) . . . . . . . . . . . . . 58

7.19 Evolution of relative change of RMSE boundary layer humidity and of soil moisture (August 2017) $\ldots \ldots \ldots$. . . . . . . . . 58

7.20 Evolution of relative change of RMSE of LST (March and August 2017) . . . . . . . . . . . . . . . . 59

7.21 Evolution of relative change of RMSE of $2 \mathrm{~m}$-temperature (March and August 2017) $\ldots \ldots \ldots \ldots \ldots$. . . . . . . . . . . . 60

7.22 Evolution of relative change of RMSE of sensible heat flux (March

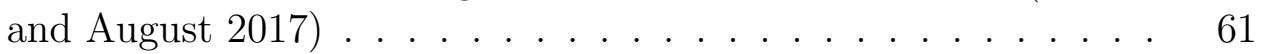

7.23 Evolution of relative change of RMSE of latent heat flux (March and August 2017) $\ldots \ldots \ldots \ldots \ldots$. . . . . . . . . . . . . 61

7.24 Evolution of relative change of RMSE of 2 m-relative humidity (March and August 2017) $\ldots \ldots \ldots \ldots 2$

7.25 Evolution of relative change of RMSE boundary layer temperature forecast and of soil temperature forecast (March 2017)] . . . 63

7.26 Evolution of relative change of RMSE boundary layer temperature forecast and of soil temperature forecast (August 2017) . . 64

$8.1 \quad$ Schematic visualization of a successful analysis step . . . . . . 67

$8.2 \quad$ Example of Lorenz 1963 system . . . . . . . . . . . . . . 71

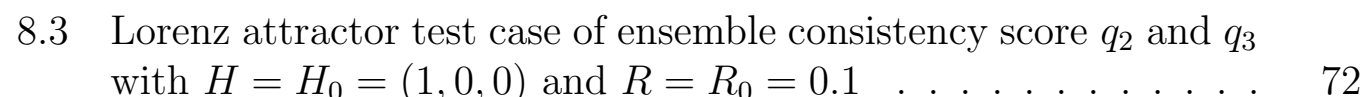

8.4 Lorenz attractor sensitivity test case of ensemble consistency score $q_{2}$ and $q_{3}$ with $\left.H=(h, 0,0), h=\{0.80,0.81, \ldots, 1.19,1.20\}\right] \quad 73$

8.5 Lorenz attractor sensitivity test case of ensemble consistency score $q_{2}$ and $q_{3}$ with $\left.R=\{0.01,0.02, \ldots, 0.29,0.30\}\right] \ldots$. . . . 74

8.6 Agreement between ensemble consistency score $q$ and impact of 7

$8.7 \quad$ Averaged correlation coefficient during March 2017 . . . . . . . 78

$8.8 \quad$ Averaged correlation coefficient during August 2017 . . . . . . . 79 
8.9 Evaluation of appropriate correlations of case studies in March and August, 2017 . . . . . . . . . . . . . . . . . . . 80

8.10 Evaluation of $q$ of different atmospheric temperature layers at August 29, 20171200 UTC and August 3020170000 UTC . . . 81

8.11 Evaluation of $q$-values at August 28, 2017 2300 UTC and August 29,20172300 UTC . . . . . . . . . . . . . . . . . 82

8.12 Evaluation of $q$ at March 27, 2017 at 0600 UTC and at 1700 UTC 82 


\section{List of Tables}

3.1 Adapted namelist setting of nature run, changes for turbulence parameterization are based on Blahak $[2015) . \ldots . . . . .219$

7.1 Experiments with different control vector settings. Table from Sgoff et al. $[(2020)] \ldots \ldots \ldots \ldots$. . . . . . . . . . . . . . 48

$8.1 \quad$ Setup of Lorenz 1963 experiments . . . . . . . . . . . . . . . . . 70

8.2 Contingency table of $q$ of the Lorenz 1963 system. . . . . . . . . 72

8.3 Namelist settings of KENDA changed due to ensemble consistency score test case. . . . . . . . . . . . 76 


\section{Bibliography}

Abdolghafoorian, A. and Farhadi, L. (2019). Estimation of Surface Turbulent Fluxes From Land Surface Moisture and Temperature Via a Variational Data Assimilation Framework. Water Resources Research, 55(6):4648-4667.

Aires, F., Prigent, C., and Rossow, W. B. (2004). Temporal interpolation of global surface skin temperature diurnal cycle over land under clear and cloudy conditions. Journal of Geophysical Research: Atmospheres, 109(D4).

Baldauf, M., Förstner, J., Klink, S., Reinhardt, T., Schraff, C., Seifert, A., and Stephan, K. (2011). Kurze Beschreibung des Lokal-Modells Kürzestfrist COSMO-DE (LMK) und seiner Datenbank auf dem Datenserver des DWD. Technical report, Deutscher Wetterdienst, Geschäftsbereich Forschung und Entwicklung: Offenbach, Germany.

Bannister, R. N. (2008a). A review of forecast error covariance statistics in atmospheric variational data assimilation. I: Characteristics and measurements of forecast error covariances. Quarterly Journal of the Royal Meteorological Society, 134(637):1951-1970.

Bannister, R. N. (2008b). A review of forecast error covariance statistics in atmospheric variational data assimilation. II: Modelling the forecast error covariance statistics. Quarterly Journal of the Royal Meteorological Society, 134(637):1971-1996.

Bateni, S., Entekhabi, D., and Jeng, D.-S. (2013a). Variational assimilation of land surface temperature and the estimation of surface energy balance components. Journal of Hydrology, 481:143 - 156.

Bateni, S. M., Entekhabi, D., and Castelli, F. (2013b). Mapping evaporation and estimation of surface control of evaporation using remotely sensed land surface temperature from a constellation of satellites. Water Resources Research, 49(2):950-968.

Berk, A., Acharya, P. K., Bernstein, L. S., Anderson, G. P., Chetwynd, J. H., and Hoke, M. L. (2000). Reformulation of the MODTRAN band model for higher spectral resolution. In Shen, S. S. and Descour, M. R., editors, Algorithms for Multispectral, Hyperspectral, and Ultraspectral Imagery VI, volume 4049, pages 190 - 198. International Society for Optics and Photonics, SPIE.

Blahak, U. (2015). Implementation and Significance of TKE-Advection in COSMO 5.0 for itype_turb=3 and Other Turbulence-Related LES-like 
Sensitivity Studies Including 3D Turbulence. COSMO Newsletter, 15(1.2):1120 .

Borbas, E., Wetzel Seemann, S., Huang, H.-L., Li, J., and Menzel, W. P. (2005). Global profile training database for satellite regression retrievals with estimates of skin temperature and emissivity. Proceedings of the International ATOVS Study conference-XIV, Bejing China, pages 763-770.

Bosilovich, M. G., Radakovich, J. D., da Silva, A., Todling, R., and Verter, F. (2007). Skin Temperature Analysis and Bias Correction in a Coupled LandAtmosphere Data Assimilation System. Journal of the Meteorological Society of Japan. Ser. II, 85A:205-228.

Bosveld, F. C., Baas, P., Steeneveld, G.-J., Holtslag, A. A. M., Angevine, W. M., Bazile, E., de Bruijn, E. I. F., Deacu, Daniel an d Edwards, J. M., Ek, M., Larson, V. E., Pleim, J. E., Raschendorfer, M., and Svensson, G. (2014). The Third GABLS Intercomparison Case for Evaluation Studies of BoundaryLayer Models. Part B: Results and Process Understanding. Boundary-Layer Meteorology, 152(2):157-187.

Boussetta, S., Koike, T., Yang, K., Graf, T., and Pathmathevan, M. (2008). Development of a coupled land-atmosphere satellite data assimilation system for improved local atmospheric simulations. Remote Sensing of Environment, $112(3): 720-734$.

Braden, H. ((reviesd version) 2012). The model AMBETI, A detailed description of a soil-plant-atmosphere model. Technical report, Deutscher Wetterdienst, Offenbach.

Burgers, G., Jan van Leeuwen, P., and Evensen, G. (1998). Analysis Scheme in the Ensemble Kalman Filter. Monthly Weather Review, 126(6):1719-1724.

Candy, B., Saunders, R. W., Ghent, D., and Bulgin, C. E. (2017). The Impact of Satellite-Derived Land Surface Temperatures on Numerical Weather Prediction Analyses and Forecasts. Journal of Geophysical Research: Atmospheres, 122(18):9783-9802.

Caselles, V., Valor, E., Coll, C., and Rubio, E. (1997). Thermal band selection for the PRISM instrument: 1. Analysis of emissivity-temperature separation algorithms. Journal of Geophysical Research, 102:11.

Desroziers, G., Berre, L., Chapnik, B., and Poli, P. (2005). Diagnosis of observation, background and analysis-error statistics in observation space. Quarterly Journal of the Royal Meteorological Society, 131(613):3385-3396.

Doms, G., Förstner, J., Heise, E., Herzog, H.-J., Mironov, D., Raschendorfer, M., Reinhardt, T., Ritter, B., Schrodin, R., Schulz, J.-P., and Vogel, G. (2011). A description of the nonhydrostatic regional COSMO model. Part II: Physical parameterization. Technical report, Deutscher Wetterdienst: Offenbach, Germany. 
Eaton, J. W., Bateman, D., Hauberg, S., and Wehbring, R. (2020). GNU Octave version 5.2.0 manual: a high-level interactive language for numerical computations.

Ehrendorfer, M. (2007). A review of issues in ensemble-based Kalman filtering. Meteorologische Zeitschrift, 16(6):795-818.

Evensen, G. (1994). Sequential data assimilation with a nonlinear quasigeostrophic model using Monte Carlo methods to forecast error statistics. Journal of Geophysical Research: Oceans, 99(C5):10143-10162.

Evensen, G. (2003). The Ensemble Kalman Filter: theoretical formulation and practical implementation. Ocean Dynamics, 53(4):343-367.

Freitas, S. C., Trigo, I. F., Bioucas-Dias, J. M., and Goettsche, F.-M. (2010). Quantifying the Uncertainty of Land Surface Temperature Retrievals From SEVIRI/Meteosat. IEEE Transactions on Geoscience and Remote Sensing, 48(1, 2):523-534.

Gaspari, G. and Cohn, S. E. (1999). Construction of correlation functions in two and three dimensions. Quarterly Journal of the Royal Meteorological Society, $125(554): 723-757$.

Ghent, D., Kaduk, J., Remedios, J., Ardö, J., and Balzter, H. (2010). Assimilation of land surface temperature into the land surface model JULES with an ensemble Kalman filter. Journal of Geophysical Research: Atmospheres, 115(D19):n/a-n/a. D19112.

Göttsche, F.-M., Olesen, F.-S., and Bork-Unkelbach, A. (2013). Validation of land surface temperature derived from MSG/SEVIRI with in situ measurements at Gobabeb, Namibia. International Journal of Remote Sensing, 34(9-10):3069-3083.

Han, X., Hendricks Franssen, H.-J., Li, X., Zhang, Y., Montzka, C., and Vereecken, H. (2013). Joint Assimilation of Surface Temperature and L-Band Microwave Brightness Temperature in Land Data Assimilation. Vadose Zone Journal, 12(3).

Harnisch, F. and Keil, C. (2015). Initial Conditions for Convective-Scale Ensemble Forecasting Provided by Ensemble Data Assimilation. Monthly Weather Review, 143(5):1583-1600.

Holtslag, A. A. M., Steeneveld, G. J., and van de Wiel, B. J. H. (2007). Role of land-surface temperature feedback on model performance for the stable boundary layer. In Atmospheric Boundary Layers: Nature, Theory and Applications to Environmental Modelling and Security, pages 205-220. Springer New York, New York, NY.

Holtslag, A. A. M., Svensson, G., Baas, P., Basu, S., Beare, B., Beljaars, A. C. M., Bosveld, F. C., Cuxart, J., Lindvall, J., Steeneveld, G. J., Tjernström, M., and Van De Wiel, B. J. H. (2013). Stable Atmospheric Boundary Layers and Diurnal Cycles: Challenges for Weather and Climate Models. Bulletin of the American Meteorological Society, 94(11):1691-1706. 
Houser, P. R., De Lannoy, G. J., and Walker, J. P. (2010). Land surface data assimilation. In Lahoz, W., Khattatov, B., and Menard, R., editors, Data Assimilation: Making Sense of Observations, pages 549-597. Springer Berlin Heidelberg, Berlin, Heidelberg.

Huang, C., Li, X., Lu, L., and Gu, J. (2008). Experiments of one-dimensional soil moisture assimilation system based on ensemble Kalman filter. Remote Sensing of Environment, 112(3):888 - 900.

Hunt, B. R., Kostelich, E. J., and Szunyogh, I. (2007). Efficient data assimilation for spatiotemporal chaos: A local ensemble transform Kalman filter. Physica D: Nonlinear Phenomena, 230(1-2):112 - 126.

Jin, M. (2004). Analysis of Land Skin Temperature Using AVHRR Observations. Bulletin of the American Meteorological Society, 85(4):587-600.

Kalman, R. E. (1960). A New Approach to Linear Filtering and Prediction Problems. Journal of Basic Engineering, 82(1):35-45.

Knuth, D. E. (1998). The art of computer programming, Volume 2, Seminumerical algorithms. Upper Saddle River, NJ, 3rd edition.

Koster, R. D., Sud, Y. C., Guo, Z., Dirmeyer, P. A., Bonan, G., Oleson, K. W., Chan, E., Verseghy, D., Cox, P., Davies, H., Kowalczyk, E., Gordon, C. T., Kanae, S., Lawrence, D., Liu, P., Mocko, D., Lu, C.-H., Mitchell, K., Malyshev, S., McAvaney, B., Oki, T., Yamada, T., Pitman, A., Taylor, C. M., Vasic, R., and Xue, Y. (2006). GLACE: The Global Land-Atmosphere Coupling Experiment. Part I: Overview. Journal of Hydrometeorology, $7(4): 590-610$.

Lin, L.-F. and Pu, Z. (2018). Characteristics of Background Error Covariance of Soil Moisture and Atmospheric States in Strongly Coupled Land-Atmosphere Data Assimilation. Journal of Applied Meteorology and Climatology, 57(11):2507-2529.

Lin, L.-F. and Pu, Z. (2019). Examining the Impact of SMAP Soil Moisture Retrievals on Short-Range Weather Prediction under Weakly and Strongly Coupled Data Assimilation with WRF-Noah. Monthly Weather Review, 147(12):4345-4366.

Lin, L.-F. and Pu, Z. (2020). Improving Near-Surface Short-Range Weather Forecasts Using Strongly Coupled Land-Atmosphere Data Assimilation with GSI-EnKF. Monthly Weather Review, 148(7):2863-2888.

Margulis, S. A. and Entekhabi, D. (2003). Variational Assimilation of Radiometric Surface Temperature and Reference-Level Micrometeorology into a Model of the Atmospheric Boundary Layer and Land Surface. Monthly Weather Review, 131(7):1272-1288.

Masutani, M., Schlatter, T., Errico, R., Stoffelen, A., Andersson, E., Lahoz, W., Woollen, J., Emmitt, G., Riishøjgaard, L.-p., and Lord, S. (2010). Observing System Simulation Experiments, pages 647-679. Springer Berlin Heidelberg. 
McNider, R. T., Song, A. J., Casey, D. M., Wetzel, P. J., Crosson, W. L., and Rabin, R. M. (1994). Toward a Dynamic-Thermodynamic Assimilation of Satellite Surface Temperature in Numerical Atmospheric Models. Monthly Weather Review, 122(12):2784-2803.

Nakamura, G. and Potthast, R. (2015). Programming of numerical algorithms and useful tools, pages 6-1 to 6-18. 2053-2563. IOP Publishing.

Noilhan, J. and Planton, S. (1989). A Simple Parameterization of Land Surface Processes for Meteorological Models. Monthly Weather Review, 117(3):536549.

NWC-SAF (2016). Algorithm Theoretical Basis Document for the Cloud Product Processors of the NWC/GEO. NWC/CDOP2/GEO/MFL/SCI/ATBD/Cloud, 1(Rev. 1):1-118.

Penny, S. G. and Hamill, T. M. (2017). Coupled Data Assimilation for Integrated Earth System Analysis and Prediction. Bulletin of the American Meteorological Society, 98(7):ES169-ES172.

Peres, L. F. and DaCamara, C. C. (2005). Emissivity maps to retrieve landsurface temperature from MSG/SEVIRI. IEEE Transactions on Geoscience and Remote Sensing, 43(8):1834 - 1844.

Pinjosovsky, H. S. B., Thiria, S., Ottlé, C., Brajard, J., Badran, F., and Maugis, P. (2017). Variational assimilation of land surface temperature within the ORCHIDEE Land Surface Model Version 1.2.6. Geoscientific Model Development, 10(1):85-104.

Pipunic, R., Walker, J., and Western, A. (2008). Assimilation of remotely sensed data for improved latent and sensible heat flux prediction: A comparative synthetic study. Remote Sensing of Environment, 112(4):1295 - 1305. Remote Sensing Data Assimilation Special Issue.

Reichle, R. H., Kumar, S. V., Mahanama, S. P. P., Koster, R. D., and Liu, Q. (2010). Assimilation of Satellite-Derived Skin Temperature Observations into Land Surface Models. Journal of Hydrometeorology, 11(5):1103-1122.

Ritter, B. and Geleyn, J.-F. (1992). A Comprehensive Radiation Scheme for Numerical Weather Prediction Models with Potential Applications in Climate Simulations. Monthly Weather Review, 120(2):303-325.

Samuelsson, P., Gollvik, S., and Ullerstig, A. (2006). The land-surface scheme of the Rossby Centre regional atmospheric climate model (RCA3). Technical Report 122, Climate research - Rossby Centre.

Sanchez, P. A., Ahamed, S., Carré, F., Hartemink, A. E., Hempel, J., Huising, J., Lagacherie, P., McBratney, A. B., McKenzie, N. J., Mendonça-Santos, M. d. L., Minasny, B., Montanarella, L., Okoth, P., Palm, C. A., Sachs, J. D., Shepherd, K. D., Vågen, T.-G., Vanlauwe, B., Walsh, M. G., Winowiecki, L. A., and Zhang, G.-L. (2009). Digital Soil Map of the World. Science, 325(5941):680-681. 
Sandu, I., Beljaars, A., Bechtold, P., Mauritsen, T., and Balsamo, G. (2013). Why is it so difficult to represent stably stratified conditions in numerical weather prediction (NWP) models? Journal of Advances in Modeling Earth Systems, 5(2):117-133.

Santanello, J. A., Kumar, S. V., Peters-Lidard, C. D., Harrison, K., and Zhou, S. (2013). Impact of Land Model Calibration on Coupled Land-Atmosphere Prediction. Journal of Hydrometeorology, 14(5):1373-1400.

Schmetz, J., Pili, P., Tjemkes, S., Just, D., Kerkmann, J., Rota, S., and Ratier, A. (2002). Supplement to an introduction to meteosat second generation (msg). Bulletin of the American Meteorological Society, 83(7):991-991.

Schraff, C., Reich, H., Rhodin, A., Schomburg, A., Stephan, K., Periáñez, A., and Potthast, R. (2016). Kilometre-scale ensemble data assimilation for the COSMO model (KENDA). Quarterly Journal of the Royal Meteorological Society, 142(696):1453-1472.

Schulz, J.-P. and Vogel, G. (2020). Improving the Processes in the Land Surface Scheme TERRA: Bare Soil Evaporation and Skin Temperature. Atmosphere, 11(5):513.

Schulz, J.-P., Vogel, G., Becker, C., Kothe, S., Rummel, U., and Ahrens, B. (2016). Evaluation of the ground heat flux simulated by a multi-layer land surface scheme using high-quality observations at grass land and bare soil. Meteorologische Zeitschrift, 25(5):607-620.

Sgoff, C., Schomburg, A., Schmidli, J., and Potthast, R. (2020). Assimilating synthetic land surface temperature into a coupled land-atmosphere system. Quarterly Journal of the Royal Meteorological Society, n/a(n/a).

Shrestha, P., Kurtz, W., Vogel, G., Schulz, J.-P., Sulis, M., Hendricks Franssen, H.-J., Kollet, S., and Simmer, C. (2018). Connection between root zone soil moisture and surface energy flux partitioning using modeling, observations, and data assimilation for a temperate grassland site in germany. Journal of Geophysical Research: Biogeosciences, 123(9):2839-2862.

Sluka, T. C., Penny, S. G., Kalnay, E., and Miyoshi, T. (2016). Assimilating atmospheric observations into the ocean using strongly coupled ensemble data assimilation. Geophysical Research Letters, 43(2):752-759.

Smith, P. J., Lawless, A. S., and Nichols, N. K. (2017). Estimating Forecast Error Covariances for Strongly Coupled Atmosphere-Ocean 4D-Var Data Assimilation. Monthly Weather Review, 145(10):4011-4035.

Smith, P. J., Lawless, A. S., and Nichols, N. K. (2018). Treating Sample Covariances for Use in Strongly Coupled Atmosphere-Ocean Data Assimilation. Geophysical Research Letters, 45(1):445-454.

Szunyogh, I., Kostelich, E. J., Gyarmati, G., Kalnay, E., Hunt, B. R., Ott, E., Satterfield, E., and Yorke, J. A. (2008). A local ensemble transform Kalman filter data assimilation system for the NCEP global model. Tellus A: Dynamic Meteorology and Oceanography, 60(1):113-130. 
Tajfar, E., Bateni, S., Lakshmi, V., and Ek, M. (2020a). Estimation of surface heat fluxes via variational assimilation of land surface temperature, air temperature and specific humidity into a coupled land surface-atmospheric boundary layer model. Journal of Hydrology, 583:124577.

Tajfar, E., Bateni, S. M., Margulis, S. A., Gentine, P., and Auligne, T. (2020b). Estimation of Turbulent Heat Fluxes via Assimilation of Air Temperature and Specific Humidity into an Atmospheric Boundary Layer Model. Journal of Hydrometeorology, 21(2):205-225.

Talagrand, O. and Courtier, P. (1987). Variational Assimilation of Meteorological Observations With the Adjoint Vorticity Equation. I: Theory. Quarterly Journal of the Royal Meteorological Society, 113(478):1311-1328.

Trigo, I. F., Boussetta, S., Viterbo, P., Balsamo, G., Beljaars, A., and Sandu, I. (2015). Comparison of model land skin temperature with remotely sensed estimates and assessment of surface-atmosphere coupling. Journal of Geophysical Research: Atmospheres, 120(23):12,096-12,111. 2015JD023812.

Trigo, I. F., Dacamara, C. C., Viterbo, P., Roujean, J.-L., Olesen, F., Barroso, C., de Coca, F. C., Carrer, D., Freitas, S. C., García-Haro, J., Geiger, B., Gellens-Meulenberghs, F., Ghilain, N., Meliá, J., Pessanha, L., Siljamo, N., and Arboleda, A. (2011). The Satellite Application Facility for Land Surface Analysis. International Journal of Remote Sensing, 32(10):2725-2744.

Trigo, I. F., Monteiro, I. T., Olesen, F., and Kabsch, E. (2008a). An assessment of remotely sensed land surface temperature. Journal of Geophysical Research: Atmospheres, 113(D17). D17108.

Trigo, I. F., Peres, L. F., DaCamara, C. C., and Freitas, S. C. (2008b). Thermal Land Surface Emissivity Retrieved From SEVIRI/Meteosat. IEEE Transactions on Geoscience and Remote Sensing, 46(2):307 - 315.

Verger, A., Camacho, F., García-Haro, F., and Meliá, J. (2009). Prototyping of Land-SAF leaf area index algorithm with VEGETATION and MODIS data over Europe. Remote Sensing of Environment, 113(11):2285 - 2297.

Verhoef, A. and Vidale, P. (2012). The role of skin layer heat transfer in the surface energy balance. In Workshop on Workshop on Diurnal cycles and the stable boundary layer, 7-10 November 2011, pages 223-233, Shinfield Park, Reading. ECMWF, ECMWF.

Verseghy, D. L., McFarlane, N. A., and Lazare, M. (1993). Class - A Canadian land surface scheme for GCMS, II. Vegetation model and coupled runs. International Journal of Climatology, 13(4):347-370.

Viterbo, P. and Beljaars, A. C. M. (1995). An Improved Land Surface Parameterization Scheme in the ECMWF Model and its Validation. Journal of Climate, 8(11):2716-2748. 
Vogel, G., Shrestha, P., Schulz, J.-P., Becker, C., and Rummel, U. (2015). Modelluntersuchungen zum Einfluss der Abschattung der Solarstrahlung durch die Vegetation auf die Erdbodentemperaturen in Falkenberg. Technical report, Deutscher Wetterdienst, Meteorologisches Observatorium Lindenberg.

Wan, Z. and Dozier, J. (1996). A generalized split-window algorithm for retrieving land-surface temperature from space. IEEE Transactions on Geoscience and Remote Sensing, 34(4):892 - 905.

Whitaker, J. S. and Hamill, T. M. (2012). Evaluating Methods to Account for System Errors in Ensemble Data Assimilation. Monthly Weather Review, 140(9):3078-3089.

Xu, T., Bateni, S. M., Neale, C. M. U., Auligne, T., and Liu, S. (2018). Estimation of Turbulent Heat Fluxes by Assimilation of Land Surface Temperature Observations From GOES Satellites Into an Ensemble Kalman Smoother Framework. Journal of Geophysical Research: Atmospheres, 123(5):2409-2423.

Yang, S.-C., Kalnay, E., Hunt, B., and E. Bowler, N. (2009). Weight interpolation for efficient data assimilation with the Local Ensemble Transform Kalman Filter. Quarterly Journal of the Royal Meteorological Society, 135(638):251-262.

Zhang, F., Snyder, C., and Sun, J. (2004). Impacts of Initial Estimate and Observation Availability on Convective-Scale Data Assimilation with an Ensemble Kalman Filter. Monthly Weather Review, 132(5):1238-1253.

Zhang, J. and Zhang, X. (2010). A soil moisture assimilation scheme using satellite-retrieved skin temperature in meso-scale weather forecast model. Atmospheric Research, 95(2):333 - 352. Special Section: Little Alaska Weather Symposium 2008.

Zhang, S., Liu, Y., and Zhang, W. (2013). Ensemble square root filter assimilation of near-surface soil moisture and reference-level observations into a coupled land surface-boundary layer model. Journal of Meteorological Research, 27(4):541-555.

Zängl, G., Reinert, D., Rípodas, P., and Baldauf, M. (2015). The ICON (ICOsahedral Non-hydrostatic) modelling framework of DWD and MPI-M: Description of the non-hydrostatic dynamical core. Quarterly Journal of the Royal Meteorological Society, 141(687):563-579. 


\title{
Deutsche Zusammenfassung
}

\author{
Assimilation synthetischer Landoberflächentemperaturen \\ in ein vollständig gekoppeltes Land-Atmosphären-Modell
}

\section{Motivation und Hinführung}

Die planetare beziehungsweise atmosphärische Grenzschicht ist die unterste Schicht unserer Atmosphäre. In diesen unteren ein bis drei Kilometern spielt sich der Großteil des irdischen Lebens ab. Das Wettergeschehen hier hat eine direkte Wirkung auf unser Leben, unsere Gesellschaft, unsere Ökonomie und unsere Energie- und Landwirtschaft. Aus diesem Grund ist die Vorhersage des Wetters in diesem Bereich der Atmosphäre von großer Bedeutung. Der solare Energieeintrag in den Boden beeinflusst die Prozesses innerhalb der Grenzschicht stark, denn die Energie wird durch die Kopplung zwischen Boden und Atmosphäre an beide weitergegeben. Dadurch hängt der Aufbau und die Entwicklung der atmosphärischen Grenzschicht wesentlich von der LandAtmosphären-Kopplung ab. Dementsprechend ist diese Kopplung ein wichtiger Prozess um die Grenzschicht in Modellen realistisch simulieren zu können.

Die Prozesse innerhalb der Atmosphäre sind nichtlinear und chaotisch. Dadurch können Abweichungen vom realen Zustand des Systems in den Anfangsbedingungen eines numerischen Wettervorhersagemodells exponentiell anwachsen. Somit limitiert unter anderem die Genauigkeit der Anfangsbedingungen des Modells den zeitlichen Vorhersagehorizont des Wettergeschehens. Um die Vorhersagbarkeit des Wetters zu verbessern, müssen zum Einen die physikalischen Prozesse der Atmosphäre im Vorhersagemodell realistisch abgebildet werden und zum Anderen der Anfangszustand des Modells möglichst genau den realen Zustand abbilden. Um die Abweichungen des Anfangszustands vom wahren Zustand zu verringern, ist es sinnvoll die Information von empirischen Beobachtungen des wahren Zustands, zum Beispiel Beobachtungen von Radiosondenaufstiegen, mit dem Zustand des Wettervorhersagemodells zu verknüpfen. Unter dem Überbegriff Datenassimilation gibt es unterschiedliche Methoden der inversen Modellierung, wie den Ensemble Kalman Filter oder die 4DVAR, um die Beobachtungen und deren Fehler mit dem Modell und dessen Fehlern zu einem verbesserten Anfangszustand zu kombinieren. Um die Simulation der Grenzschicht zu verbessern, hat demnach die Assimilation von bodennahen Beobachtungen wie der Landoberflächentemperatur (engl. land surface temperature, LST), großes Potential. Die LST ist eine Beobachtung direkt an der Grenze zwischen Atmosphäre und Land und enthält somit indirekt auch Informationen über den Zustand der unteren Atmosphäre und über 
den Zustand der oberen Bodenschichten. Um diese Information der LST in Land und Atmosphäre einzubringen, werden in dieser Arbeit zwei Ansätze untersucht: die schwache und die stark gekoppelte Datenassimilation. In einem schwach gekoppelten Assimilationssystem wird die Beobachtung im Assimilationsschritt nur in eines der beiden Systeme des Modells, Atmosphäre oder Boden direkt eingebracht. Erst durch die darauf folgende Vorhersage im gekoppelten Modell wird die Information im gesamten Modellsystem verteilt. In einem stark gekoppelten Assimilationssystem dagegen wird die Information bereits im Assimilationsschritt in beide Systeme, Land und Atmosphäre direkt eingebracht.

Um den Einfluss der Assimilation von LST auf die atmosphärische Grenzschicht abschätzen zu können, wurden im Rahmen dieser Doktorarbeit mehrere zweitägige Experimente in März und August 2017 auf der Basis von simulierten LST-Beobachtungen durchgeführt (engl. Observing System Simulation Experiment, OSSE). Um die synthetischen Beobachtungen der LST zu generieren, wurde zunächst ein sogenannter Nature Run aufgesetzt. Dieser hochaufgelöste Modelllauf wird im OSSE als wahrer Zustand der Atmosphäre und des Bodens angenommen. Er wird dazu verwendet die Beobachtungen der LST zu generieren und auch zur Evaluierung der Ergebnisse verwendet. Das Assimilationssystem dieser Arbeit basiert auf einem lokalen transformierten Ensemble-Kalman-Filter. Dadurch hängt der Einfluss der Assimilation von LST auf die Modellvariablen der Atmosphäre und des Bodens stark von den Kovarianzen innerhalb des Modellensembles ab. Um diese Kovarianzen genauer $\mathrm{zu}$ untersuchen und zu überprüfen, ob sie mit dem Verhalten der Wahrheit zusammen passen, wurde ein Ensemble-Konsistenz-Score $q$ entwickelt.

\section{Datenssimilation: KENDA}

Als Assimilationssystem wurde das Ensemble basierte Datenassimilationssystem für regionale Wettervorhersagemodelle des Deutschen Wetterdienstes (Kilometre-scale ensemble based data assimilation, KENDA) verwendet. Es basiert auf dem lokalen transformierten Ensemble-Kalman-Filter (engl. Local ensemble transform Kalman filter, LETKF). Der Zyklus des Assimilationssystems besteht aus einem Vorhersageschritt und einem Analyseschritt. Im Vorhersageschritt wird eine einstündige Kurzvorhersage simuliert, der sogenannte first guess. Dieser first guess ist die Modellinformation, welche im Analyseschritt mit den Beobachtungen kombiniert wird. Im Analyseschritt werden allerdings nicht alle Modellvariablen durch den Assimilationsalgorithmus aktualisiert, sondern nur ein Teil der prognostischen Modellvariablen. Diese werden im Kontrollvektor zusammengefasst und aus ihnen werden auch die Modellfehlerkovarianzen bestimmt. Um das vollständig gekoppelte Datenassimilationssystem möglich zu machen, wurde der Kontrollvektor um die prognostischen Modellvariablen des Bodens, Bodentemperatur und -feuchte, erweitert. Dadurch werden im Analyseschritt die Modellvariablen des Kontrollvektors über die LandAtmosphären-Grenze hinweg angepasst. Das Modellensemble besteht aus 40 Membern. Durch die begrenzte Anzahl der Member können Schätzfehler in den Ensemblekovarianzen auftreten. Damit Beobachtungen die Analyse dadurch 
nicht verschlechtern, wird ihr räumliches Einflussgebiet in Atmosphäre und Boden über Gaspari-Cohn Korrelationsfunktionen festgelegt. Diese Lokalisierung wurde an die LST-Beobachtungen angepasst und für den Boden neu implementiert.

\section{Nature Run}

Der Nature Run ist ein wichtiger Bestandteil des OSSE. Er wird als Wahrheit angenommen und sollte deshalb das Verhalten der realen Atmosphäre wiedergeben können. In dieser Arbeit wurde für den Nature Run und den Assimilationszyklus das langjährig operationelle numerische Wettervorhersagemodell des Deutschen Wetterdienstes verwendet: das COSMO-Modell (engl. Consortium for small-scale modelling, COSMO). Der Nature Run wurde dabei mit einer horizontalen Auflösung von $0,7 \mathrm{~km}$ simuliert, während für den Assimilationszyklus ein Ensemble mit 2,8 km horizontaler Aufösung simuliert wurde. Um der höheren Auflösung Sorge zu tragen, wurde im Nature Run die Strahlung alle drei statt alle 15 Minuten simuliert und auch das Turbulenzschema an die hohe Auflösung angepasst. Da zwei Fallstudien in März und August evaluiert wurden, wurden auch zwei Nature Runs durchgeführt. Der Nature Run im Frühjahr ist eine freie Vorhersage vom 25. bis 29. März 2017 und der Nature Run im Sommer ist eine freie Vorhersage vom 26. bis 30. August 2017. Diese Zeiträume wurden wegen ihrer vergleichsweisen geringen Bewölkung gewählt, da die LST Beobachtungen aus bodennahen Satellitenbeobachtungen abgeleitet werden, welche nur in wolkenfreien Gebieten gemessen werden können. Ein Gebiet um Lindenberg, eine Messstation des Deutsche Wetterdienstes südöstlich von Berlin, wurde als Modellgebiet gewählt. Somit ist das Gebiet weitestgehend flach und deckt Norddeutschland und kleine Abschnitte von Polen und der Tschechischen Republik ab.

\section{Landoberflächentemperatur als Beobachtung und im Modell}

Eines der existierenden LST Retrievals wird von der Einrichtung zur Anwendung von Satellitendaten mit der Ausrichtung zur Analyse der Landoberfläche (engl.: Land Surface Analysis Satellite Application Facility, Land-SAF) bereitgestellt. Die dafür benötigten bodennahen Messungen stammen von einem Imager für Strahlung im sichtbaren und infraroten Spektralbereich (engl.: Spinning enhanced visible and infrared imager, SEVIRI), welcher sich an Bord des geostationären Satelliten „Meteosat Second Generation“ befindet.

Die Auswertung dieses LST Retrievals im Vergleich mit dem COSMOModell Version 5.04c zeigt, dass der Tagesgang der LST im Modell zu schwach ausgeprägt ist. Ein Grund dafür ist die fehlende Vegetationstemperatur im COSMO-Modell. Um den Tagesgang besser abzubilden, wurden zwei Parametrisierungen der Vegetationstemperatur getestet. Die eine Parametrisierung basiert auf einem Vegetationsmodell mit prognostischer Vegetationstemperatur und die andere Parametrisierung auf der Einführung 
einer dünnen Schicht zwischen Boden und Atmosphäre, deren Leitfähigkeit die Effekte der Vegetation auf die Temperatur abbildet. Im Vergleich der beiden Parametrisierungen mit den realen Beobachtungen schnitt das Leitfähigkeitsschema besser ab, da nur es in der Lage war auch nachts den stärkeren Tagesgang der Beobachtungen abzubilden. Für die Dissertation wurde deshalb diese Parametrisierung gewählt. Seit 2020 ist sie auch Teil des Modells im operationellen Betrieb des DWD.

\section{Assimilation der Landoberflächentemperatur}

Anhand der Assimilation einzelner LST-Beobachtungen am Tag und in der Nacht wurde die technische Umsetzung des stark gekoppelten Assimilationssystems überprüft. Dabei zeigte sich, dass sowohl die Kopplung von Land und Atmosphäre im Analyseschritt als auch die zusätzlich implementierte Lokalisierung im Boden funktionieren.

Um das gekoppelte Assimilationssystem zu testen, wurden mehrere zweitägige Experimente in März und August 2017 durchgeführt. In den Experimenten wurden stündlich alle synthetisch erzeugten LST-Beobachtungen assimiliert. Die Experimente unterschieden sich in der Umsetzung der Kopplung des Assimilationssystems zwischen Land und Atmosphäre. Sowohl im März als auch im August wurden zwei Experimente mit schwacher Kopplung durchgeführt. In dem ersten Experiment enthielt der Kontrollvektor nur atmosphärische Variablen und in dem zweiten Experiment nur Bodenvariablen. Das dritte Experiment verwendete das stark gekoppelte Assimilationssystem, in welchem der Kontrollvektor beides, Atmosphären- und Bodenvariablen enthielt.

Die Assimilation der LST beeinflusste in allen drei Experimenten hauptsächlich die Temperatur der Grenzschicht und des Bodens, aber auch deren Feuchte und die turbulenten Flüsse. Die Wirkung der LST-Assimilation war in den schwach gekoppelten Systemen immer in dem Teil des Modells, Atmosphäre oder Boden, stärker, welcher direkt durch die Assimilation beeinflusst wurde. Durch die starke Kopplung des Assimilationssystems konnten die Effekte der schwach gekoppelten Systeme kombiniert werden und somit der Einfluss der Assimilation von LST optimal genutzt werden.

Da die thermischen Prozesse der Atmosphäre schnelllebiger sind als die des Bodens, bleibt der Effekt der LST-Assimilation im Boden auch länger sichtbar als in der Atmosphäre. Sobald weniger Beobachtungen assimiliert werden, zum Beispiel auf Grund von zunehmender Bewölkung, nimmt der Einfluss der LSTAssimilation auf die Modellvariablen der Atmosphäre ab. Die Bodenvariablen dagegen können die Informationen länger im Modell halten und profitieren somit auch länger von der LST-Assimilation. Die Assimilation der LST konnte die mittleren quadratischen Fehler (engl. Root mean square error, RMSE) der vorhergesagten Temperaturen in Grenzschicht und Boden reduzieren. Vor allem tagsüber profitierte die Grenzschichttemperatur von der Assimilation der LST. Mit dem vollständig gekoppelten System wurde dabei die größte Reduktion des 
RMSE erreicht.

Ausgehend von den Analysen des gekoppelten Assimilationssystems wurde alle sechs Stunden eine freie Vorhersage mit 24 Stunden Vorhersagehorizont simuliert. Ein Vergleich dieser Vorhersagen mit Vorhersagen ohne LSTAssimilation zeigt, dass der RMSE der Grenzschichttemperatur durch die Assimilation von LST-Beobachtungen in den ersten drei bis neun Vorhersagestunden reduziert werden kann. Der RMSE der Bodentemperatur reduziert sich sogar über den kompletten Vorhersagezeitraum. Von der länger anhaltenden Verbesserung im Boden, kann auch die Grenzschichttemperatur noch nach 15 Vorhersagestunden profitieren, obwohl der direkte Effekt der LST-Assimilation auf die Atmosphäre bereits geendet hat.

Während die Temperaturen des Land-Atmosphären Modells von der LSTAssimilation weitgehend profitierten, erhöhte sich teilweise der RMSE in der spezifischen Feuchte der Grenzschicht. Auch auf die Bodenfeuchte hatte die Assimilation von LST, zum Beispiel am zweiten Tag des Augustexperiments, einen negativen Einfluss. Ein möglicher Grund dafür sind inkonsistente Kovarianzen der Modellfehler. Durch diese Kovarianzen verteilt der Algorithmus des LETKF die Information der Beobachtung im Modellraum. Sie werden aus dem Modellensemble bestimmt. Passen sie nicht zu den wahren Kovarianzen, hier die des Nature Runs, kann die Assimilation die Anfangsbedingungen auch verschlechtern.

\section{Ensemble-Konsistenz-Score}

Um einen besseren Einblick in die Kovarianzen der Modellfehler der OSSE zu bekommen, wurde im Rahmen dieser Arbeit der Ensemble-Konsistenz-Score $q$ entwickelt. Der Score ist ein Schätzer für die Konsistenz der EnembleKovarianzen. Sein Vorzeichen gibt an, ob die Ensemblekovarianzen zwischen der beobachteten Variable und jeweils einer weiteren Modellvariable zum Nature Run passen oder nicht. Ist $q>0$, dann passt das Ensemble zum Nature Run und sorgt dafür, dass der LETKF das Modell näher an die Wahrheit zieht. Ist $q \leq 0$, dann passt das Ensemble nicht zum Nature Run und der LETKF kann im Analyseschritt das Modellensemble von dem wahren Zustand der Atmosphäre beziehungsweise des Bodens weg ziehen. Berechnet wird der Score $q$ für jeden Gitterpunkt des Analysegitters auf dem eine Beobachtung liegt.

Getestet wurde der Score $q$ zunächst für ein Lorenz 1963 Modellsystem. Dabei wurde der Einfluss des Beobachtungsoperators $H$ und der Beobachtungsfehlerkovarianzmatrix $R$ auf die Zuverlässigkeit der Schätzung von $q$ untersucht. Sowohl die Berechnung des Scores $q$ als auch der Analyseschritt der Assimilation hängen von $H$ und $R$ ab. Abweichungen der beiden Größen zwischen der Berechnung von $q$ und dem Assimilationsverfahren können sich also auf die Übereinstimmung von Vorzeichen des Scores $q$ und dem Ergebnis des Analyseschritts auswirken. Sensitivitätstests von $H$ und $R$ ergaben, dass Abweichungen von $R$ zwischen Analyseschritt und Berechnung des Scores $q$ deutlich weniger ins 
Gewicht fallen, als ein nicht übereinstimmender Beobachtungsoperator.

Um den Score $q$ auf die Assimilation von LST im gekoppelten LandAtmosphäre-Modell anwenden zu können, wurden deshalb die Beobachtungen und das Analysegitter auf das Modellgitter verschoben. Darüber hinaus wurden die horizontale und vertikale Lokalisierung so gewählt, dass immer nur eine Beobachtung die angrenzenden Modellvariablen im Analyseschritt beeinflussen konnte.

Ein erster Blick auf die Ensemblekovarianzen der Fallstudien in März und August 2017 zeigte starke Korrelationen zwischen der LST und den Temperaturen der untersten Atmosphärenschicht und der obersten Bodenschicht. Die Korrelationen zwischen der LST und der Bodenfeuchte der obersten Bodenschicht und der LST und der spezifischen Feuchte der untersten Atmosphärenschicht waren weniger stark ausgeprägt. Während die Bodenfeuchte nachts eine geringe Antikorrelation zur der LST aufwies, waren die LST und die spezifische Feuchte den ganzen Tagesverlauf über schwach positiv korreliert. Die Auswertung des Scores $q$ zeigte darüber hinaus, dass die Korrelationen zwischen den Temperaturen, im März vor allem bei Nacht und im August vor allem bei Tag gut zum Verhalten des Nature Run passen. Die Korrelationen der Feuchtevariablen dagegen werden vom Ensemble weniger deutlich reproduziert und entsprechen eher einem Rauschen. Dieses Rauschen kann zu einem unpassenden Einfluss der LST Assimilation auf diese Variablen führen, was auch die OSSE Experimenten zeigten. Das führte zu der Schlussfolgerung, dass die teils negative Wirkung der LST Assimilation auf die Feuchtevariablen tatsächlich auf unpassende Ensemblekorrelationen zurückzuführen ist.

\section{Schlussfolgerungen}

Die durchgeführten Fallstudien in März und August 2017 zeigen, dass sowohl der first guess als auch die 24 Stunden Vorhersagen der Grenzschichttemperatur und Bodentemperatur von der Assimilation von LST profitieren können und Experimente mit realen LST-Beobachtungen ein nächster Schritt sind. Die Wirkung der LST-Assimilation auf die atmosphärische Grenzschicht hängt dabei vom Tagesgang, der Anzahl der verfügbaren synthetischen Beobachtungen und der Art der Kopplung des Assimilationssystems ab. Je mehr LST-Beobachtungen zur Verfügung stehen, desto ausgeprägter ist der Einfluss der Assimilation. Besonders deutlich sieht man das an der Wirkung auf die Atmosphäre. Der Boden kann die gewonnene Information der LST-Assimilation länger halten, da seine Prozesse deutlich träger sind als die der Atmosphäre. Im stark gekoppelten System ist der positive Einfluss auf Grenzschichttemperatur und Bodentemperatur am größten. Die Vorhersage der Feuchtevariablen des Bodens und der Atmosphäre hingegen können sich durch die Assimilation von LST auch verschlechtern. Gleiches gilt für die Temperaturen oberhalb der nächtlichen Grenzschicht. Durch zusätzliche Assimilation von Feuchtebeobachtungen und eine angepasste vertikale Lokalisierung in der Nacht könnte in Zukunft diesen Problemen entgegen gewirkt werden. Die durchgeführten Experimente sind 
sowohl räumlich als auch zeitlich limitiert, sodass zukünftige Experimente auf einem größeren Gebiet und auch über längere Zeiträume ausgewertet werden sollten.

Zum besseren Verständnis des teils negativen Einflusses der LSTAssimilation auf Feuchte und Temperatur wurde die Konsistenz der Ensemblekorrelationen mit dem Nature Run untersucht. Dabei zeigte sich, dass die Temperaturen stärker linear mit der LST-Beobachtung korreliert sind und das diese Korrelation sowohl im Ensemble als auch im Nature Run zu finden sind. Die lineare Korrelation zwischen LST und den Feuchtevariablen ist schwächer. Darüber hinaus zeigt der Ensemble-KonsistenzScore $q$, dass die Korrelationen des Ensembles weniger gut zu denen des Nature Run passen. Eine weitere Untersuchung der Korrelationen und ihrer Abbildung in der Modellfehlerkovarianzmatrix ist ein wichtiger nächster Schritt. Können die Korrelationen zwischen Temperatur und Feuchte besser in den Analyseschritt eingehen, könnte das die Vorhersage der atmosphärischen Grenzschicht verbessern. 